Portland State University

PDXScholar

Spring 5-15-2013

\title{
Nonlinearity Analysis and Predistortion of 4G Wireless Communication Systems
}

Xiao Li

Portland State University

Follow this and additional works at: https://pdxscholar.library.pdx.edu/open_access_etds

Part of the Systems and Communications Commons Let us know how access to this document benefits you.

\section{Recommended Citation}

$\mathrm{Li}$, Xiao, "Nonlinearity Analysis and Predistortion of 4G Wireless Communication Systems" (2013). Dissertations and Theses. Paper 992.

https://doi.org/10.15760/etd.992

This Dissertation is brought to you for free and open access. It has been accepted for inclusion in Dissertations and Theses by an authorized administrator of PDXScholar. Please contact us if we can make this document more accessible: pdxscholar@pdx.edu. 
Nonlinearity Analysis and Predistortion of 4G Wireless Communication Systems

by

Xiao Li

A dissertation submitted in partial fulfillment of the requirements for the degree of

\author{
Doctor of Philosophy \\ in \\ Electrical and Computer Engineering
}

Dissertation Committee:
Fu Li, Chair
James Morris
Xiaoyu Song
Fei Xie

Portland State University

2013 


\begin{abstract}
The nonlinearity of RF power amplifiers (PA) is one of critical concerns for RF designers because it causes spectral regrowth related to in-band and out-of-band spurious emissions control in communication standards. Traditionally, RF power amplifiers must be backed off considerably from the peak of their power level in order to prevent spectral regrowth. The digital predistortion (DPD) technique is being widely used for compensation of the nonlinearity of RF power amplifiers, as the high power efficiency becomes increasely important in wireless communication systems.
\end{abstract}

However, the latest generations of communication systems, such as Wi-Fi, WiMAX, and LTE, using wider bandwidth have some additional memory distortion, other than the traditional memoryless distortion. The distortion caused by memory effect makes the traditional predistorter not precise any more for the PA linearization.

In this dissertation, the traditional prediction of 3rd order memoryless spectrum regrowth is applied to $4 \mathrm{G}$ communication signals in terms of the 3rd intercept point of PAs, based on the previous researches in Portland State University led by Professor Fu Li. Then, the spectrum regrowth prediction is extended to an arbitrarily high order with intercept points of an RF power amplifier. A simple predistortion method which enables 
direct calculation of the predistorter coefficients from the intercept points is also proposed.

Furthermore, the memory effect is taken into account for both PA modeling and predistortion. A simplified Hammerstein structure based method is proposed to analyze the nonlinear characteristic of PAs more precisely and completely. By applying the inverse structures of the PA model, the proposed predistorter corrects both the traditional memoryless nonlinear distortions and the memory effect that may exist in RF power amplifiers. The order of nonlinearity and depth of memory of a predistorter can be chosen from 0 to any arbitrarily high number. This increases the flexibility for designers to decide how to linearize power amplifier effectively and efficiently. 


\section{Acknowledgements}

It is very fortunate for me to meet and work with so many talented people during my five years in Portland; I cannot make this dissertation possible without their supports.

First and foremost, I would like to thank my advisor, Dr. Fu Li for introducing me to the field of wireless communication, providing me with guidance and suggestions for my research, spending time with me in VIP lab almost every single day. I always admired him as Sir Alex Ferguson to me.

I would like to thank my dissertation committee members, Dr. James Morris, Dr. Xiaoyu Song, and Dr. Fei Xie for serving on my committee. The special appreciation is also expressed to Dr. Heng Xiao, Dr. Chunming Liu, Dr. Qiang Wu, and Dr. Kwok-Wai Tam for their help on my research over the past years. I also benefit a lot from my internship at Tektronix, and I would like to thank my project manager Li Cui for his help.

My special thanks to my friends in Portland: Bosi Chen, Yansheng Li, Yafei Yang, Yang Shi, Xiaolong Cheng, Ming Shen, and Peng Gao. My special thanks also to my friends in Shenyang: Yuanqi Shan, Yinan Chen, Le Yang, Shixin Zhan, Song Hu, Haibo Liu, Lilin Wang, Leilei Zhen, Jin Wang, Bo Yang, Bin Huang, Fei Wang, and their spouses.

This dissertation is dedicated to my family with special thanks to my parents, the four Zeng sisters, my brother Yingtong Liu, and my sister Jingxue Yang, the days with you are my best time in US. Last but not least, I would like to thank my girlfriend, Ying, for her love, encouragement, and support. 


\section{Table of Contents}

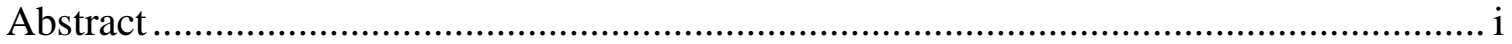

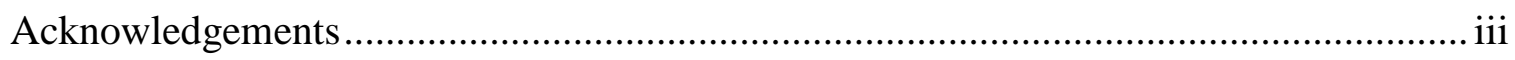

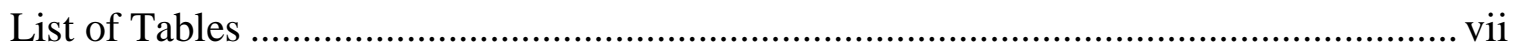

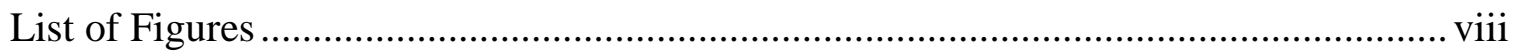

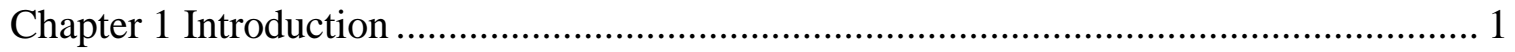

1.1 Legacy Statement of the Problems ............................................................... 1

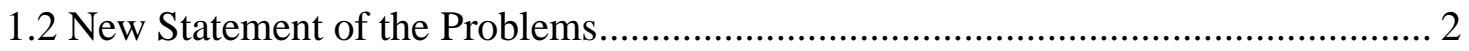

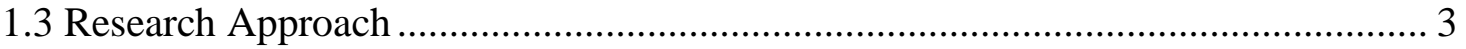

1.4 Six Contributions of This Dissertation ............................................................. 4

1.4.1 Low Order Spectrum Regrowth Prediction in 4G Communication Systems .... 5

1.4.2 Obtaining Polynomial Coefficients from Intercept Points of RF Power

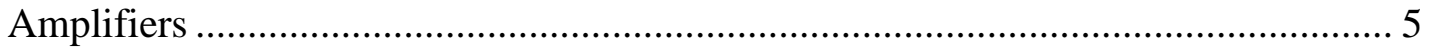

1.4.3 Explicit Expression of High Order Spectrum Regrowth in 4G Communication

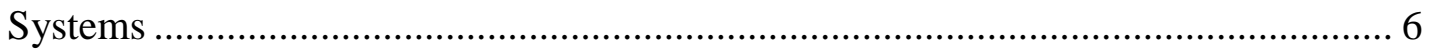

1.4.4 Nonlinear Modeling with Memory Effect .................................................... 7

1.4.5 High Order Inverse Polynomial Predistortion for Memoryless RF Power

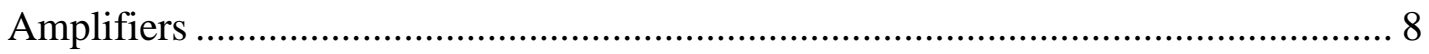

1.4.6 Predistortion with Memory Effect .......................................................... 9

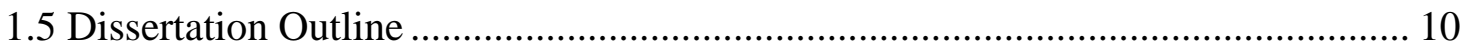

Chapter 2 Nonlinearity of Power Amplifiers............................................................... 12

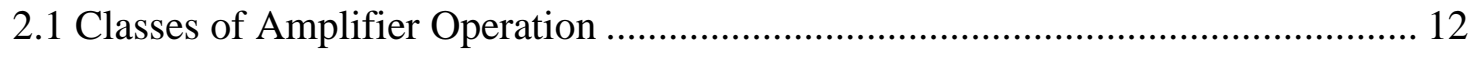


2.2 Traditional Memoryless Nonlinearity .......................................................... 15

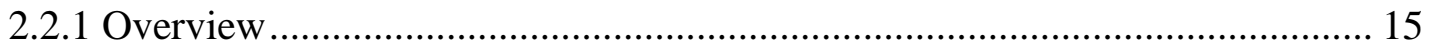

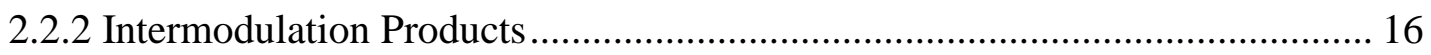

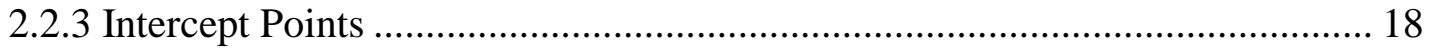

2.2.4 The $1 \mathrm{~dB}$ Compression Point ....................................................................... 24

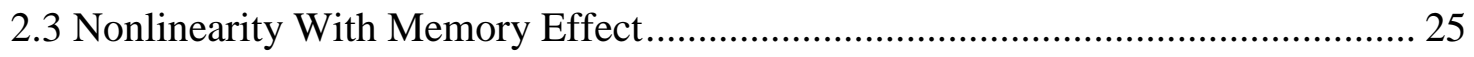

Chapter 3 Modeling and Spectrum Regrowth Analysis of Power amplifiers.................... 31

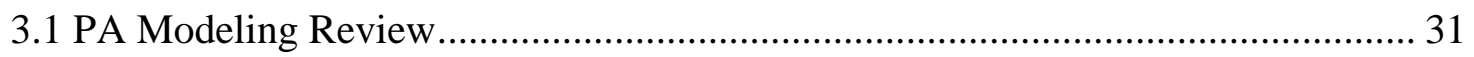

3.1.1 Memoryless Model and Quasi-memoryless Model ........................................ 32

3.1.2 Models with Memory Effect ........................................................................... 33

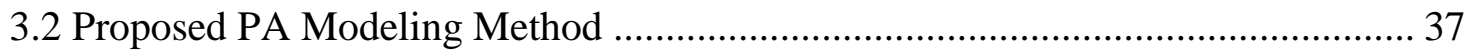

3.2.1 The Nonlinearity Part of Model ................................................................. 38

3.2.2 The Memory Effect Part of Model.................................................................. 44

Chapter 4 Spectrum Regrowth Analysis of 4G Communication Signals ........................ 47

4.1 The Equivalent Mathematical Model of LTE and WiMAX.................................... 48

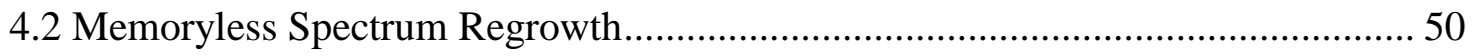

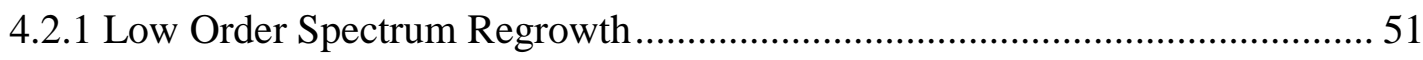

4.2.2 High Order Spectrum Regrowth ................................................................. 53

4.3 Spectrum Regrowth with Memory Effect................................................................ 59

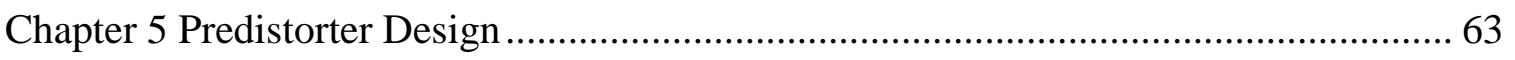

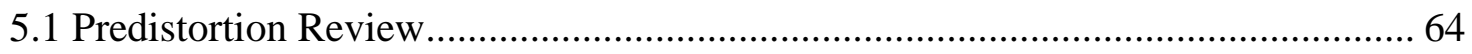

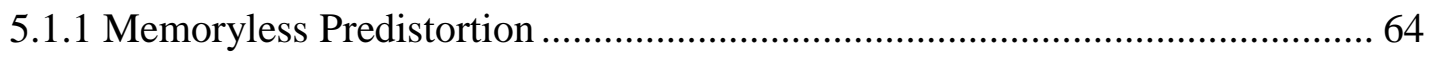

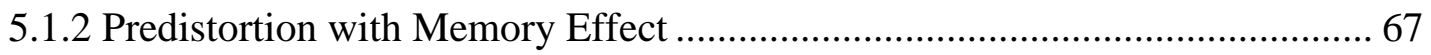




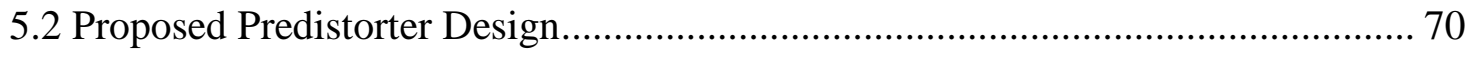

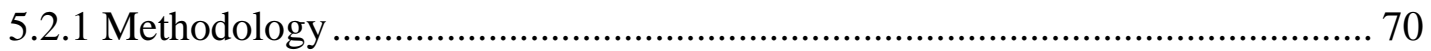

5.2.2 Predistortion of Memoryless Nonlinear Part ................................................... 71

5.2.3 Predistortion of the Memory Part ……………................................................... 77

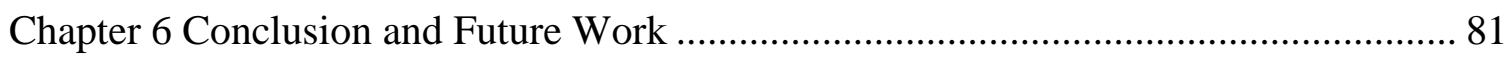

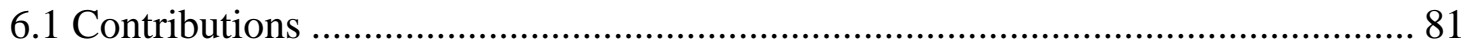

6.1.1 Low Order Spectrum Regrowth Prediction in 4G Communication Systems .. 81

6.1.2 Obtain Polynomial Coefficients from Intercept Points of RF Power Amplifiers

6.1.3 Explicit Expression of High Order Spectrum Regrowth in 4G Communication

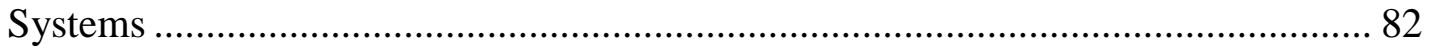

6.1.4 Nonlinear Modeling with Memory Effect …………………………….......... 83

6.1.5 High Order Inverse Polynomial Predistortion for Memoryless RF Power

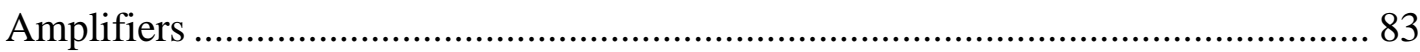

6.1.6 Predistortion with Memory Effect ………………........................................ 84

6.2 Suggestions for Future Research ..................................................................... 84

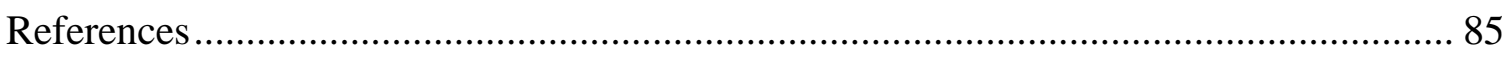

Appendix A Proof of the White Gaussian OFDM Theorem in 4.2.2 …......................... 92 


\section{List of Tables}

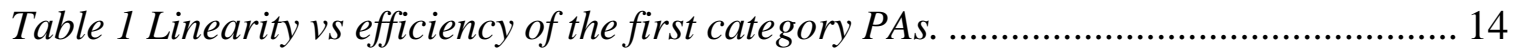

Table 2 The categories of PA models ........................................................................... 31

Table 3 The parameters used in math model for $10 \mathrm{MHz}$ data rate ............................... 49 


\section{List of Figures}

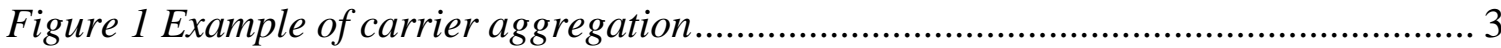

Figure 2 Learning architecture for the predistorter ..................................................... 4

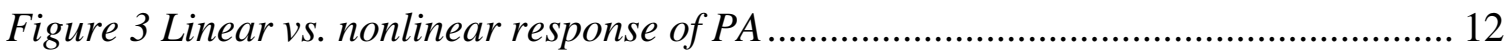

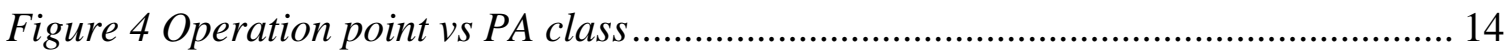

Figure 5 Harmonic distortion in a single-tone system ............................................. 15

Figure 6 Intermodulation products in a two-tone system .............................................. 18

Figure 7 Frequency presentation of each distortion term ........................................... 22

Figure 8 The definition of the $n$-th order intercept point ............................................. 24

Figure 9 The definition of the $1 \mathrm{~dB}$ compression point ............................................... 25

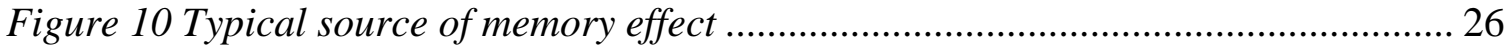

Figure 11 Spectrum regrowth with memory effect ..................................................... 27

Figure 12 AM to AM nonlinearity, scatter due tomemory effect ................................... 29

Figure 13 AM to PM nonlinearity, scatter due to memory effect .................................... 29

Figure 14 AM/AM under a one-carrier and a four-carrier WCDMA input signals, scatter

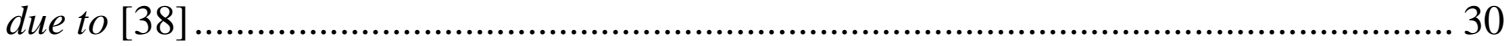

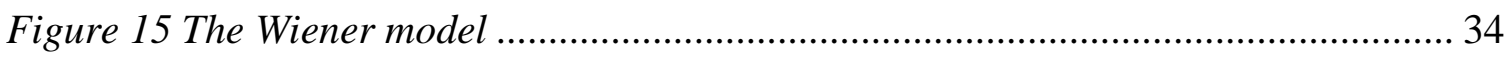

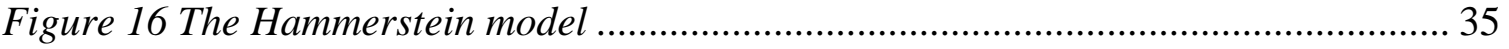

Figure 17 The Wiener-Hammerstein model ............................................................ 36

Figure 18 The Hammerstein-Wiener model ............................................................. 36

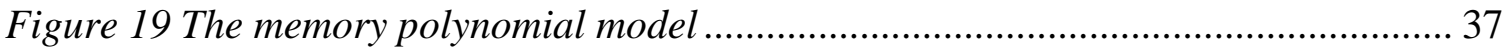

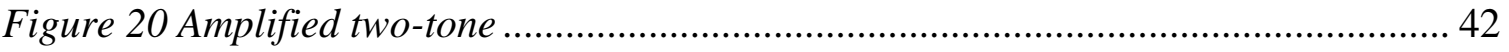


Figure 21 Amplified 1.4 MHz LTE downlink signal .... 43

Figure 22 Spectrum of the Intermodulation product of each individual term in (3.9) ..... 44

Figure 23 Power spectrum comparison of downlink LTE and WiMAX signals .............. 50

Figure 24 Power spectrum comparison of amplified downlink LTE and WiMAX signals53

Figure 25 Reverse-link CDMA signal possibility density function ................................ 54

Figure 26 Theoretical spectrum vs estimated spectrum in (4.8) ................................. 57

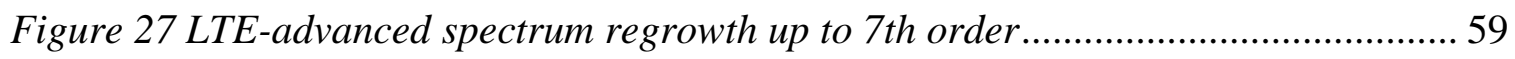

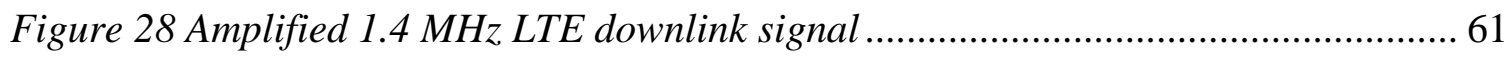

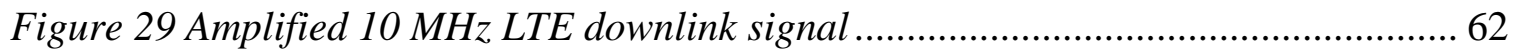

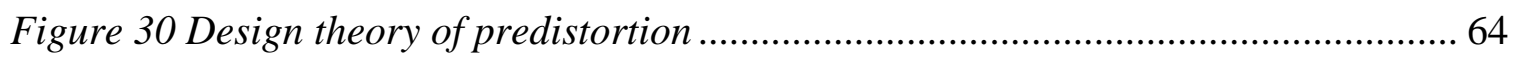

Figure 31 The direct learning architecture for the predistorter.................................... 68

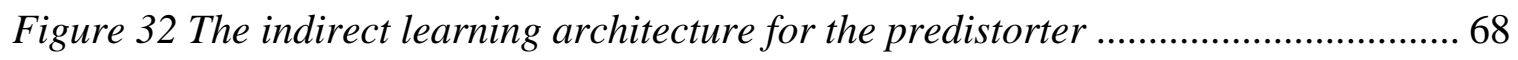

Figure 33 Predistorter learning structure …....................................................... 71

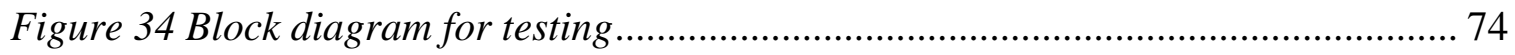

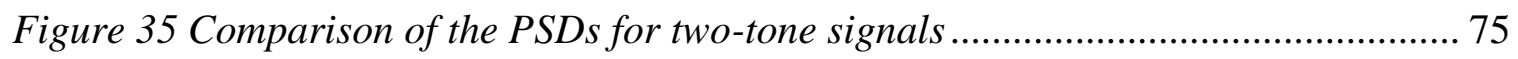

Figure 36 Comparison of the PSDs for LTE signals ............................................... 76

Figure 37 Comparison of the constellation before and after DPD(The phase offset and

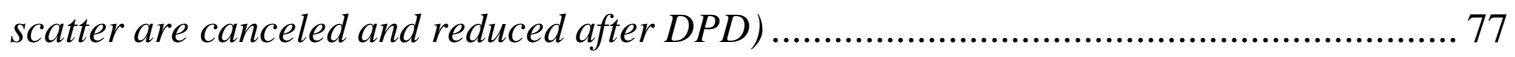

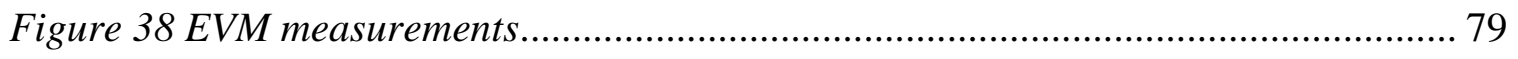

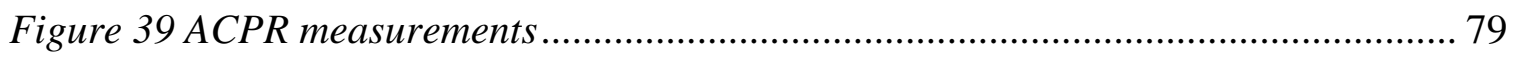

Figure 40 Power Spectrum of downlink LTE advanced signals ................................... 80 


\section{Chapter 1 Introduction}

\subsection{Legacy Statement of the Problems}

In wireless communication systems, a RF power amplifier (PA) is one very important component. One of the main concerns in RF power amplifier design is its nonlinearity. It degrades the quality of the transmitted signal and increases the interference to the adjacent channels in communication systems. Transitionally, nonlinearity is described by the nonlinear parameters such as intercept points included in the technical specifications. The coefficients of memoryless power amplifier models are directly related to the intercept points of the power amplifiers. In this case at a certain instant the amount of amplitude (AM/AM conversion) and phase distortion (AM/PM conversion) depends only on the input signal level at that time instant.

The digital predistortion (DPD) technique is widely used for compensation for the nonlinearity of RF power amplifiers. The principle of predistortion is to distort the input signal prior to amplification in the opposite way of amplifier's nonlinearity, to gain linearly amplified signals at the output. It combines two nonlinear systems to obtain a linear one. Predistorter generates intermodulation products (IMPs) that are antiphase with the IMPs in the PA, to cancel spectral regrowth. Predistortion can be realized with analog or digital electronics. Digital predistortion is more flexible and promising than analog solutions. Digital signal processor (DSP) is used for calculation of the predistortion inverse function and for the adaptation algorithms. The Look-Up Table 
(LUT) based algorithms [1] [2] and the inverse polynomial algorithms [3] [4] are two key algorithms for memoryless PA models.

\subsection{New Statement of the Problems}

The latest generation wireless communication systems, such as Wi-Fi, WiMAX and LTE use much wider bandwidth. This creates new problems for the analysis of RF power amplifiers because the memory effect is directly associated with the bandwidth.

Long term evolution (LTE) is a next generation mobile communication system, as a project of the 3rd Generation Partnership Project (3GPP). Worldwide Interoperability for Microwave Access (WiMAX) is another emerging wireless technology that provides high speed mobile data and telecommunication services based on IEEE 802.16 standards. Both LTE and WiMAX support frequency division duplexing (FDD) and time-division duplexing (TDD) modes, and have more deployment flexibility than the previous $3 G$ systems by using scalable channel bandwidths with different numbers of subcarriers while keeping frequency spacing between subcarriers constant. An important requirement for $4 \mathrm{G}$ mobile systems is that they can support very high peak data rates for mobile users, up to $1 \mathrm{~Gb} / \mathrm{s}$ in static and pedestrian environments, and up to $100 \mathrm{Mb} / \mathrm{s}$ in a high-speed mobile environment [5]. Therefore, the focus of 3GPP is now gradually shifting towards the further evolution of LTE, Release 10, referred to as LTEAdvanced. Carrier aggregation supporting up to 5 component carriers for both downlink 
and uplink, is of the most distinct feature of LTE-Advanced. LTE-Advanced supports wider transmission bandwidths than the maximum $20 \mathrm{MHz}$ bandwidth specified in 3GPP Release 8/9 [6]. Bandwidths up to $100 \mathrm{MHz}$ are foreseen to provide peak data rates up to $1 \mathrm{~Gb} / \mathrm{s}$ as shown in Figure 1.

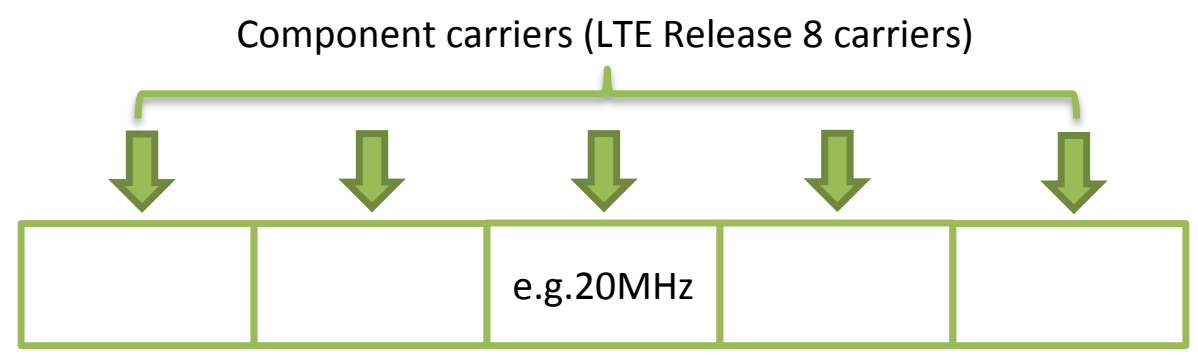

Five $20 \mathrm{MHz}$ component carriers => Total $100 \mathrm{MHz}$ bandwidth

Figure 1 Example of carrier aggregation

As the result, for systems with wide bandwidth and larger memory, the AM/AM and AM/PM characteristics do not contain complete information about the nonlinearity. Therefore, the accuracy of this model is reduced. This memory effect brings additional distortion to PAs and the traditional memoryless predistorter is not enough to linearize them.

\subsection{Research Approach}

In this dissertation, we propose a DPD learning scheme in Figure 2. $x(n)$ is the input signal, and $y(n)$ is the amplified output signal of a power amplifier. It is modeled by two blocks: a memoryless nonlinear block (A) and a LTI (linear time invariant) block (C) 
presenting memory effect; The digital Predistorter is modeled by two inverse functions according to PA model: inverse nonlinear block (B) and an inverse LTI block (D). The inverse nonlinear block (B) compensates the memoryless distortion of the PA modeled by block (A), and the inverse LTI block (D) cancels the memory effect presented by block (C).

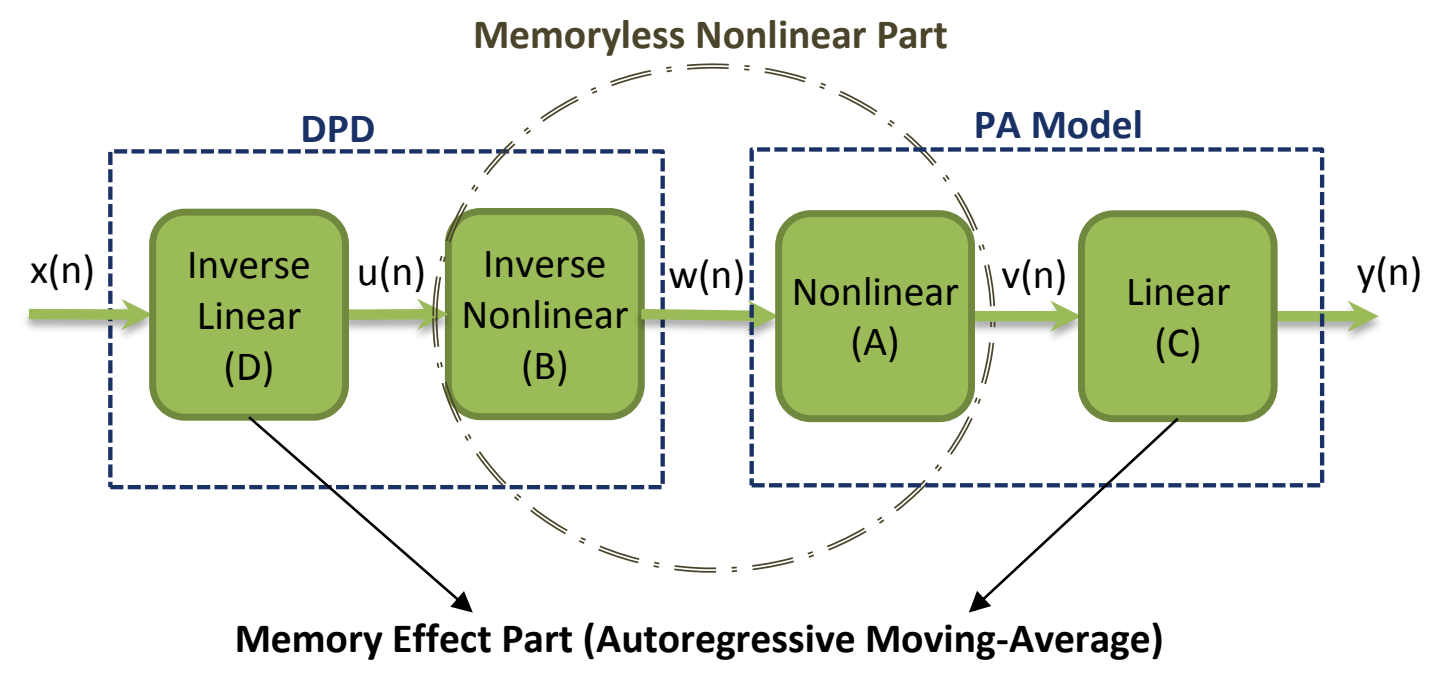

Figure 2 Learning architecture for the predistorter

\subsection{Six Contributions of This Dissertation}

This dissertation provided a systematic method to analyze and correct the nonlinearity of RF power amplifiers. The six contributions are listed as below. 


\subsubsection{Low Order Spectrum Regrowth Prediction in 4G Communication Systems}

Based on the relationship between the third order intercept point $\left(I P_{3}\right)$ and the third order polynomial coefficient of an RF power amplifier, our group has previously analyzed and predicted the spectrum regrowth caused by the nonlinearity of RF power amplifiers in relation to their intermodulation parameters for two OFDM based signals: Wi-Fi and digital broadcasting [7]. This technology is now extended to LTE and WiMAX signals in this dissertation and verified by the validity of the theoretical result derived by experimental data. This provides insight for designing power amplifiers and digital predistorters in terms of out-band spectrum regrowth. (See Chapter 4.2.1)

Related work: The research team led by $\mathrm{Dr}$. Fu $\mathrm{Li}$, has analyzed the memoryless spectrum regrowth of an RF power amplifier up to 5th order in CDMA, TDMA, Wi-Fi, TDSCDMA systems [8] [9] [7] [10]. However, there is no expression for the spectrum regrowth in $4 \mathrm{G}$ communication systems, in terms of the power amplifier parameters, such as intercept points.

\subsubsection{Obtaining Polynomial Coefficients from Intercept Points of RF Power Amplifiers}

The expression of the polynomial coefficient from the intercept point is generalized to arbitrarily high order. With this result, the memoryless polynomial of each RF power amplifier can be determined from its intercept points or intermodulation products completely and hence could be used for the design of power amplifiers or predistorters. 
(See Chapter 3.2.1)

Related work: The mathematical relationship between the 5th order coefficients of polynomial model and intercept points of power amplifiers can be seen in our earlier published work, such as [11]. However, there is no generic mathematical relationship derived between the coefficients of polynomial model and higher order intercept points. We generalized this relationship by expanding it up to an arbitrarily high $n$-th order, such that the nonlinear polynomial coefficient for any orders can be obtained from the intercept points of RF power amplifiers.

\subsubsection{Explicit Expression of High Order Spectrum Regrowth in 4G Communication}

\section{Systems}

To study the nonlinearity of RF power amplifiers, we showed that LTE-Advanced signals with large number of subcarriers can be expressed as a white Gaussian noise statistical model with a flat power spectrum. This characteristic enables us to express the spectrum regrowth of LTE-Advanced signals up to an arbitrarily high order in an explicit form, in terms of the traditional PA nonlinearity parameter intercept points without complicated convolution. (See Chapter 4.2.2)

Related work: [12] [13] [14] indicates that an OFDM signal can be considered as Gaussian distributed if the amplitude of the subcarriers are independent and identically 
distributed random variables when the number of subcarriers is large enough. Even though this is an old result, but nobody has proved it.

We proved that the statistical model of CDMA signal converges to a white Gaussian random process as the number of chips goes to infinity [15]. Based on the rectangular pulse shaping filter of CDMA signals, the authors derived the explicit expression of spectrum regrowth up to an arbitrarily high order in terms of polynomial coefficients of PA [16]. However, there is no this kind of explicit expressions of spectrum regrowth for OFDM based LTE or LTE advanced signals.

\subsubsection{Nonlinear Modeling with Memory Effect}

The latest generation wireless communication systems with wider bandwidth, such as Wi-Fi, WiMAX, and LTE, create additional distortion to PAs which affect the performance of digital predistortion (DPD) design. We proposed a simplified Hammerstein structurebased method to analyze the nonlinear characteristic of PAs with consideration of the memory effect. The simplified method produced more accurate results and reduced the complexity of the classic Hammerstein system identification at the same time. (See Chapter 3.2.2)

Related work: There are a number of mathematical models to describe the nonlinearity of PAs with memory effect. Most of them are based on the Volterra series [17], includes 
the Wiener [18], or Hammerstein [19] polynomials, and the like. The Volterra series is the most general polynomial type of nonlinearity with memory, but it requires huge effort to extract the coefficients when the order of the model increases above the third order. Hammerstein and Wiener models are the specialized version with the least number of coefficients, but are by no means the easiest to identify. The memory polynomial model [20] using the diagonal kernels of the Volterra series is widely used since its parameters can be easily estimated using least-square criteria. However, this model cannot address the memoryless nonlinearity and memory effect of PA separately, so the complexity of the system is significant when PA shows little memory effect. For most applications, if the intermodulation products are delayed as the same time, the coefficient matrix of the whole PA model is 1 , so this memory polynomial model is unnecessary to be used.

\subsubsection{High Order Inverse Polynomial Predistortion for Memoryless RF Power}

\section{Amplifiers}

In some applications, there are advantages in using predistorters to linearize PAs before compensating for the memory effect. We presented a polynomial predistortion method based on pth-order inverse method to compensate the memoryless nonlinearity of PAs. The coefficients of the polynomial predistorters up to the arbitrarily high orders can be identified directly from coefficients of the simple polynomial PA model up to arbitrarily 
high orders, which makes the compensation process much simpler than using complex algorithms computations. (See Chapter 5.2.2)

Related work: The Look-Up Table (LUT) based algorithms [2] [21] [22] and the inverse polynomial algorithms [23] [24] [25] are two key algorithms for memoryless models. To get an acceptable accuracy, the memory size of the Look-Up Table has to be larger which requires a great deal of expensive silicon area. Additionally, the corresponding tanning time is another major drawback [24]. The theory of pth-order inverse of nonlinear systems [3] was originally used for the compensation of the nonlinearities of power amplifiers with memory by a Volterra series model, but this method is unrealistically complicated when a high order of nonlinearity is taken into account and is unnecessary if the memory effect of a PA is not strong. Sunmin Lim and Changsoo Eun [4] proposed a predistorter for memoryless PAs using a pth-order inverse method, but due to mathematical complexity, only up to 9th order coefficients of the predistorter are given based on a polynomial model of PA up to 9th order.

\subsubsection{Predistortion with Memory Effect}

We proposed a predistortion method which uses an inverse polynomial to linearize nonlinearity effect. It also uses an inverse Autoregressive Moving-Average (ARMA) model to remove memory effect of RF power amplifiers. With this approach, power 
amplifiers can be predistorted by simply choosing the nonlinearity or memory depth at any arbitrary high orders. (See Chapter 5.2.3)

Related work: Two categories are considered for synthesizing the predistortion function. The first one is an indirect learning model [17]. However, two drawbacks affect the performance of the indirect learning model [26]. First, the measurement of PA's output could be noisy, thus, the adaptive algorithm converges to biased values. Second, the nonlinear filters cannot be commuted, i.e., the identified adaptive inverse model is actually a post-inverse model. Placing a copy of this model in front of a nonlinear device does not guarantee a good pre-inverse model for the nonlinear device. The authors in [27] compared the indirect and direct learning predistortion methods, and shown that the direct learning architecture achieves a better performance in almost all cases. The second one is direct learning model [20] [26], however, the inversion of the nonlinear system could be very hard to identifying if the nonlinear model is complicated or the parameters are difficult to be acquired.

\subsection{Dissertation Outline}

This dissertation is organized as outlined below:

Chapter 2: Nonlinearity of Power Amplifiers. The nonlinearity of RF power amplifiers is discussed using the principal intermodulation products. The intercept points are introduced to describe those intermodulation products. 
Chapter 3: Modeling and Spectrum Regrowth Analysis of Power amplifiers. Different mathematical models of RF power amplifiers are discussed. A new model that calculates both the memoryless nonlinearity and memory effects from PAs' interception points is proposed.

Chapter 4: The spectrum regrowth of $4 \mathrm{G}$ communication systems are analyzed using different PA models. The comparison between PAs with and without memory is discussed.

Chapter 5: Predistortion design. By applying the inverse structures of the PA model presented in Chapter 3, a new predistorter is proposed to correct the traditional memoryless nonlinear distortion and the memory effect separately. The experimental result is provided to verify the algorithm.

Chapter 6: Conclusion and future work. A summary of all the results included in this dissertation is provided. Further research works and approaches are also discussed. 


\section{Chapter 2 Nonlinearity of Power Amplifiers}

Generally speaking, a practical amplifier is only a linear device in its linear region, meaning that the output of the amplifier will not be exactly a scaled copy of the input signal when the amplifier works beyond the linear region as Figure 3 . The cause of the nonlinearity is the transistor, which in general is a nonlinear element, approximately linear only for weak signals. Nonlinearities are not readily apparent because the intermodulation products are significantly below the noise floor as a result of relatively weak carrier signals, but this effect becomes apparent when the incident power is raised above $30 \mathrm{dBm}$.
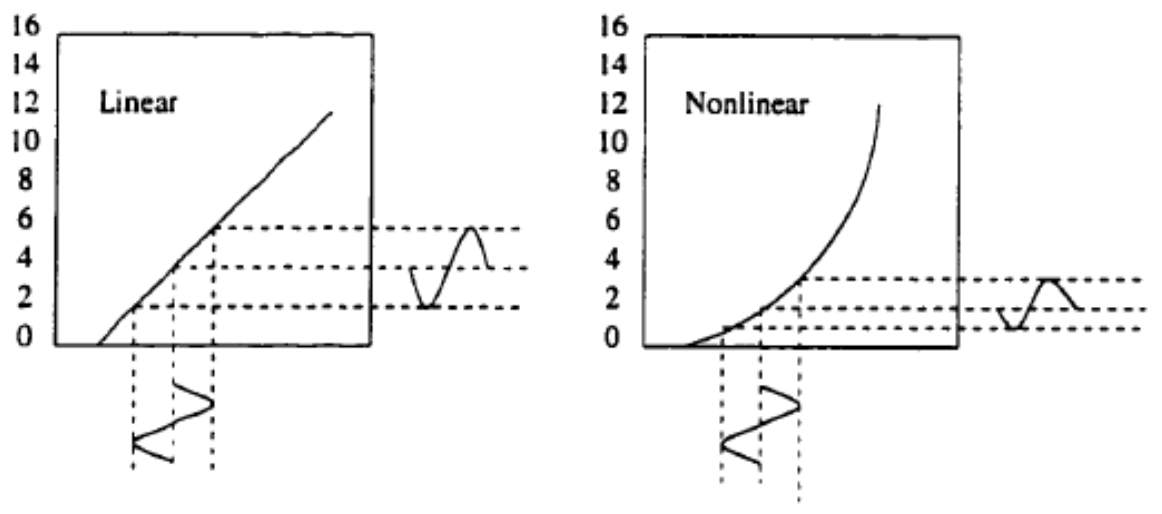

Figure 3 Linear vs. nonlinear response of PA

\subsection{Classes of Amplifier Operation}

The linearity and efficiency are the most important characteristics for the power amplifiers in communication systems. Higher linearity leads to poor efficiency and vice 
versa. Therefore, achieving a balance between the two is very important in power amplifier design.

Linear amplification is required when the signal contains AM - Amplitude Modulation or a combination of both, Amplitude and Phase Modulation (SSB, TV video carriers, QPSK, QAM, and OFDM). Signals such as CW, FM or PM have constant envelopes (amplitudes) and therefore do not require linear amplification [28].

The Efficiency of an RF power amplifier is a measure of its ability to convert the DC power from the supply into the signal power delivered to the load, and the definition can be presented as [28]

$$
\eta=\frac{\text { Signal power delivered to load }}{D C \text { power supplied to output circuit }}
$$

Depending on application requirements for linearity and efficiency, the PA operation classes can be divided into two categories [29]:

1) High linear amplifiers that are usually used in communication application (ClassA, Class-B, Class-AB, and Class-C).

2) High efficiency amplifiers that are usually used in satellite application (Class-D, Class-E, Class-F, and others). They are also known as switch mode (digital) amplification. 
Only the first category, which is usually used in mobile and microwave application, is considered in this chapter. Choosing the bias points of an RF power amplifier can determine the level of performance ultimately possible with that PA. The transistor operation points of power amplifiers from each class are plotted in Figure 4.

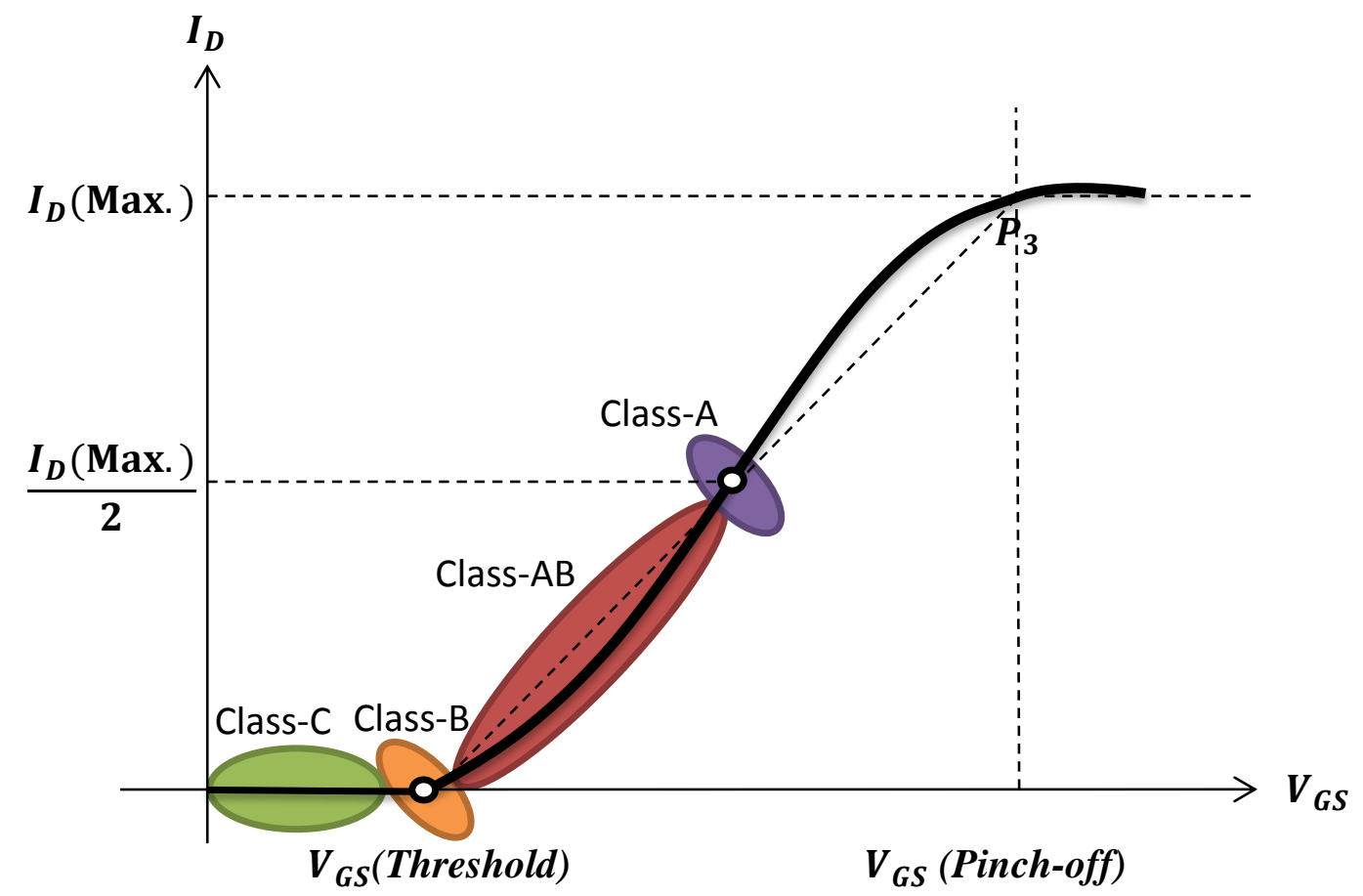

Figure 4 Operation point vs PA class

The Table 1 summarizes the linearity and efficiency of the first category PAs.

\begin{tabular}{|c|c|c|c|}
\hline & Linearity & $\begin{array}{c}\text { Maximum } \\
\text { Efficiency }\end{array}$ & Conduction Angle \\
\hline Class-A & Good & $50 \%$ & $360^{\circ}$ \\
\hline Class-AB & Intermediate & $60 \%$ & $180^{\circ}-360^{\circ}$ \\
\hline Class-B & Poor & $75 \%$ & $180^{\circ}$ \\
\hline Class-C & The poorest & $85 \%$ & $<180^{\circ}$ \\
\hline
\end{tabular}

Table 1 Linearity vs efficiency of the first category PAs. 


\subsection{Traditional Memoryless Nonlinearity}

\subsubsection{Overview}

The nonlinearity of power amplifiers (PA) degrades the signal quality and increases the interference to the adjacent channels in communication systems. One type of traditional nonlinear distortion is caused by harmonics of signals. A Single-tone test could be used to measure the nth order harmonic distortion $\left(H D_{n}\right)$. Usually, the total harmonic distortion (THD), is defined as the power sum of signals at all harmonic frequencies to the power of the signal at the fundamental frequency as [30]

$$
T H D=\frac{P_{2}{ }^{2}+P_{3}{ }^{2}+\cdots+P_{n}{ }^{2}}{P_{t}{ }^{2}}
$$

where $P_{t}$ is the power of signal at the fundamental frequency, $P_{n}$ presents the $\mathrm{nth}$ order harmonic distortion.

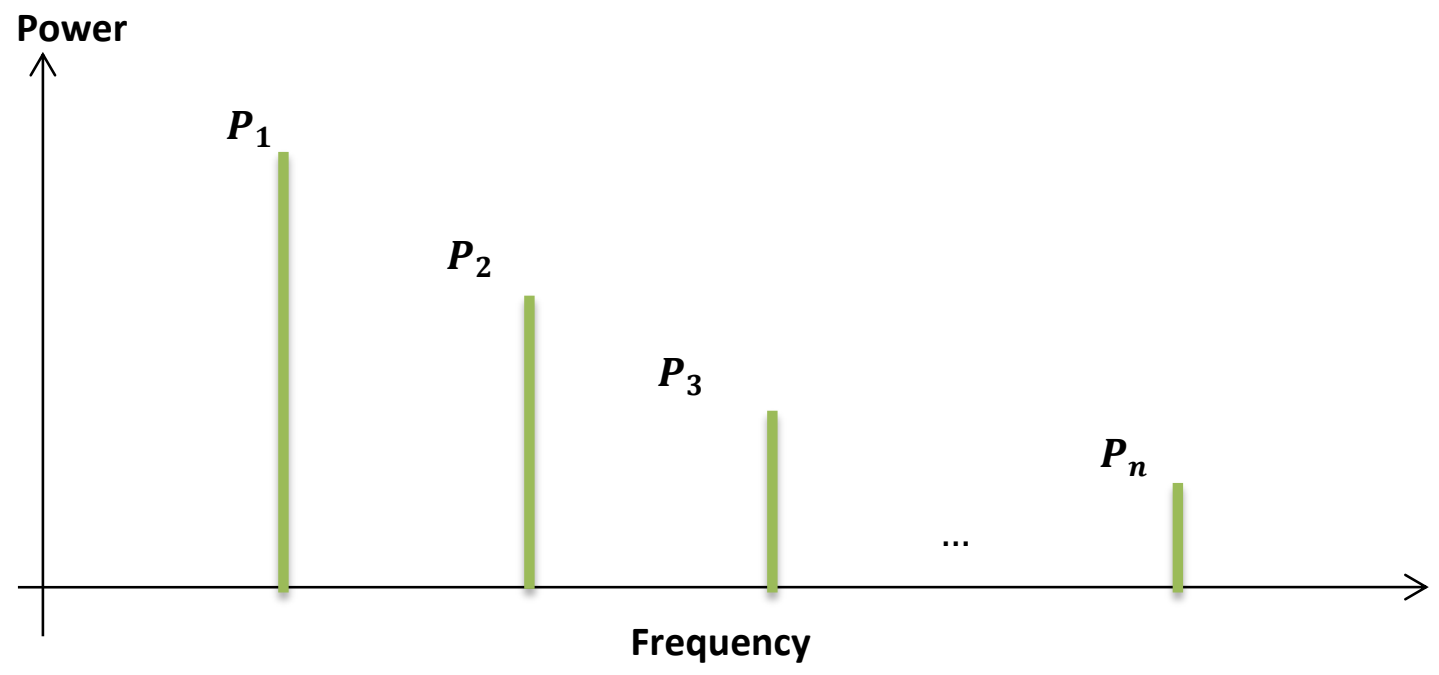

Figure 5 Harmonic distortion in a single-tone system 
Another type of traditional distortion is intermodulation products usually measured by a two-tone test. This is the major problem in communication systems, since the frequencies of some intermodulation products fall close to the fundamental signals and cannot be removed by filters.

\subsubsection{Intermodulation Products}

When the nonlinearity within power amplifiers generate other frequencies, it is known as intermodulation products $\left(I M P_{n}\right.$, where the subscript $n$ denotes the order of the intermodulation products). Intermodulation deteriorates or limits the ability of the service providers to operate at optimal performance levels and ultimately may cause subscribers to experience poor call quality.

The intermodulation between each frequency component will form additional signals at frequencies other than the harmonic frequencies. It generates signals at the sum and difference frequencies of the original frequencies and at the multiples of the sum and the difference of these frequencies.

For a linear power amplifier, the relationship between its input and output is

$$
V_{\text {out }}(t)=a V_{\text {in }}(t)
$$

where $V_{\text {out }}(t)$ is the output signal, $V_{\text {in }}(t)$ is the input signal, and $a$ is the gain of an RF Power Amplifier. 
For practical amplifiers, the output saturates at a certain value as the input amplitude is increased. The Taylor series [31] is a widely used polynomial model for PAs, and it is usually used to describe the concept of the intermodulation products. Further, practical amplifiers can have a nonlinear output-to-input characteristic modeled by Taylor's expression such that

$$
\begin{aligned}
V_{\text {out }}(t)=A\left[V_{\text {in }}(t)\right] & =\sum_{m=0}^{M} a_{m} V_{\text {in }}{ }^{m}(t) \\
& =a_{0}+a_{1} V_{\text {in }}(t)+a_{2}{V_{\text {in }}}^{2}(t)+a_{3} V_{\text {in }}{ }^{3}(t)+\cdots+a_{M} V_{\text {in }}{ }^{M}(t)
\end{aligned}
$$

Here, $a_{0}$ is the output DC offset term, and $a_{1} V_{\text {in }}(t)$ is the linear term. $a_{2} V_{\text {in }}{ }^{2}(t)$ is the second order term, and $a_{3} V_{\text {in }}{ }^{3}(t)$ is the third order term. The power amplifier will have nonlinear distortion if $a_{2}, a_{3}, \cdots, a_{M}$ are not all zero. A good linear amplifier has $a_{1}$ substantially larger than $a_{2}, a_{3}, \cdots, a_{M}$. The intermodulation of a two-tone system is shown in Figure 6. More detail will be discussed with the intercept points in 2.1.2. 


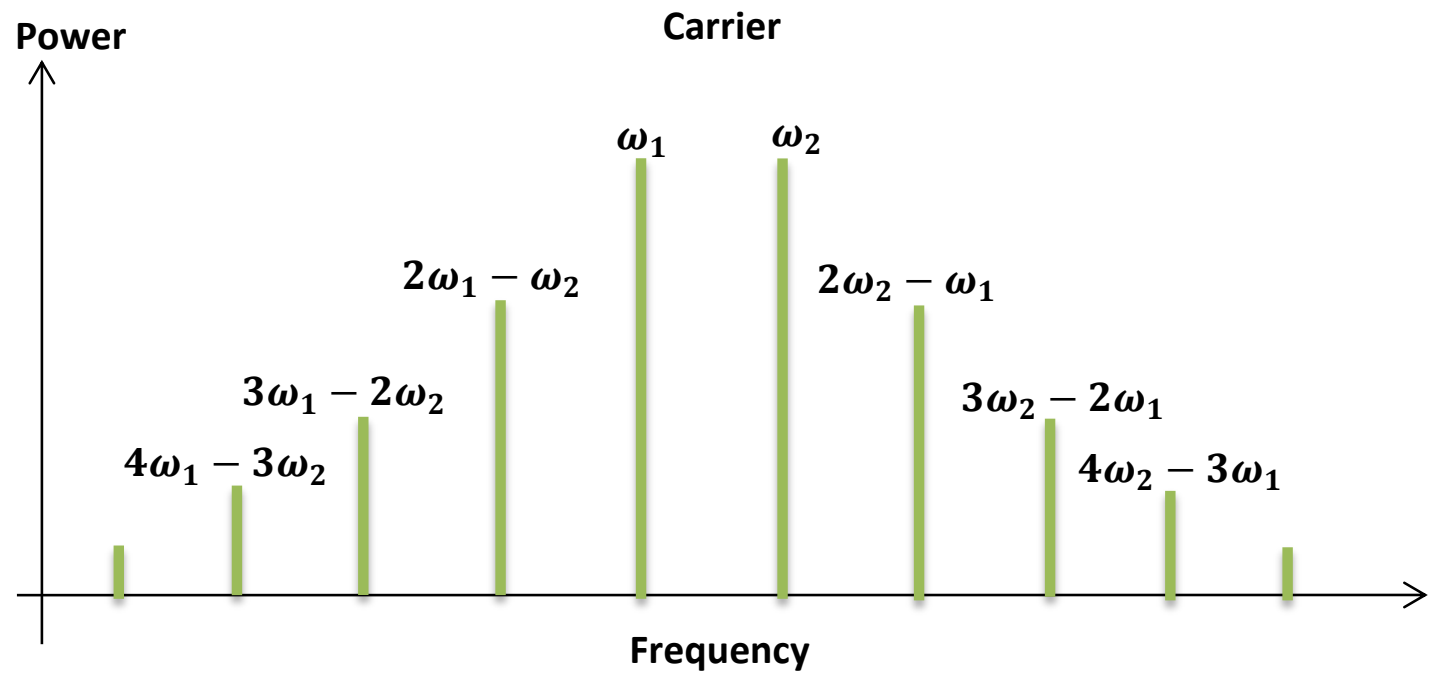

Figure 6 Intermodulation products in a two-tone system

\subsubsection{Intercept Points}

A polynomial model using Taylor series is a very simple memoryless PA model for nonlinearity analysis and digital predistortion (DPD) [31] [32]. Its coefficients should be identified directly from the intercept points of PAs, which describe the nonlinearity.

The Taylor series can be written as [31]:

$$
\begin{aligned}
v(n)=A[w(n)] & =\sum_{\substack{m=1 \\
\text { is odd }}}^{M} a_{m} w^{m}(n) \\
& =a_{1} w(n)+a_{3} w^{3}(n)+\cdots+a_{M} w^{M}(n),
\end{aligned}
$$


where $w(n)$ is the input signal of PAs, and $v(n)$ presents the amplified signal. $A$ denotes the nonlinear amplifier. Only the odd-order terms are considered in this calculation, since even-order terms at least one carrier frequency away from the center of the passband can be easily filtered out [11]. $a_{n}$ 's are the nonlinear polynomial coefficients of PA.

The intercept point is defined as the output power level at which the power of intermodulation product would intercept with the output power at fundamental frequency. Traditionally, the nonlinearity of an RF amplifier is described by the 3rd order intercept point $\left(I P_{3}\right)$ usually given in the manufacturer's datasheets. For a PA with gain compression, the output using polynomial PA model can be expressed as a function of the gain, intercept points, and the input signal. The higher order intercept points are not generally provided, but they can easily be calculated from the higher order intermodulation products (IMP) by using a two-tone test [33].

A two-tone test is used to obtain intercept points as [33]:

$$
I P_{n}=\frac{n P_{t}-I M P_{n}}{n-1}
$$

where $P_{t}$ is the power of original tone signals at the output of PAs, and $I M P_{n}$ is the power of $\mathrm{n}$-th order intermodulation product, which can be easily measured from twotone test without loss of generality, because a multi-tone signal can decompose into pairs of two-tones that all yield the same result. 
Theoretically, an input two-tone signal at two frequencies $\omega_{1}$ and $\omega_{2}$ can be written as

$$
w(t)=A\left(\cos \omega_{1} t+\cos \omega_{2} t\right)
$$

If only the 3rd order intercept point is considered, the amplified two-tone signal is

$$
\begin{aligned}
v(t)= & a_{1} w(t)+a_{2} w^{2}(t)+a_{3} w^{3}(t) \\
= & a_{1} A\left(\cos \omega_{1} t+\cos \omega_{2} t\right) \\
& +a_{2} A^{2}\left(\cos \omega_{1} t+\cos \omega_{2} t\right)^{2} \\
& +a_{3} A^{3}\left(\cos \omega_{1} t+\cos \omega_{2} t\right)^{3} \\
= & a_{1} A\left(\cos \omega_{1} t+\cos \omega_{2} t\right) \\
& +a_{2} A^{2}\left(\cos ^{2} \omega_{1} t+2 \cos \omega_{1} t \cdot \cos \omega_{2} t+\cos ^{2} \omega_{2} t\right) \\
& +a_{3} A^{3}\left(\cos ^{3} \omega_{1} t+3 \cos { }^{2} \omega_{1} t \cdot \cos \omega_{2} t+3 \cos \omega_{1} t \cdot \cos ^{2} \omega_{2} t+\cos ^{3} \omega_{2} t\right) \\
& +\frac{6 \cos \omega_{2} t+9 \cos \left(2 \omega_{1}+\omega_{2}\right)+9 \cos \left(2 \omega_{1}-\omega_{2}\right)}{4} \\
& +a_{3} A^{3}\left[\frac{3 \cos _{1} \omega_{1} t+\cos ^{3} \omega_{1} t}{4}\right. \\
= & a_{1} A\left(\cos \omega_{1} t+\cos \omega_{2} t\right) \\
& +a_{2} A^{2}\left[\frac{1+\cos 2 \omega_{1} t}{2}+\cos _{1} t+9 \cos \left(2 \omega_{2}+\omega_{1}\right)+9 \cos \left(2 \omega_{2}-\omega_{1}\right)+\cos \left(\omega_{2}-\omega_{1}\right)+\frac{1+\cos _{2} 2 \omega_{2} t}{2}\right.
\end{aligned}
$$




$$
\left.+\frac{3 \cos \omega_{2} t+\cos 3 \omega_{2} t}{4}\right]
$$

If the each term in equation (2.10) is categorized as the order of distortion, we have:

The 1st order Terms:

$$
\left(a_{1} A+\frac{9}{4} a_{3} A^{3}\right) \cos \omega_{1} t+\left(a_{1} A+\frac{9}{4} a_{3} A^{3}\right) \cos \omega_{2} t
$$

The 2nd order Terms:

$$
a_{2} A^{2}+\frac{a_{2}}{2} A^{2} \cos 2 \omega_{1} t+\frac{a_{2}}{2} A^{2} \cos 2 \omega_{2} t+a_{2} A^{2} \cos \left(\omega_{1}-\omega_{2}\right)+a_{2} A^{2} \cos \left(\omega_{1}+\omega_{2}\right)
$$

The 3rd order Terms:

$$
\begin{aligned}
& \frac{a_{3} A^{3} \cos 3 \omega_{1} t}{4}+\frac{3 a_{3} A^{3} \cos \left(2 \omega_{1}-\omega_{2}\right)}{4}+\frac{3 a_{3} A^{3} \cos \left(2 \omega_{2}-\omega_{1}\right)}{4} \\
& +\frac{3 a_{3} A^{3} \cos \left(2 \omega_{1}+\omega_{2}\right)}{4}+\frac{3 a_{3} A^{3} \cos \left(2 \omega_{2}+\omega_{1}\right)}{4}+\frac{a_{3} A^{3} \cos 3 \omega_{2} t}{4}
\end{aligned}
$$

Those terms are plotted in Figure 7. From the frequency presentation of each distortion term, harmonics (at $2 \omega_{1}, 2 \omega_{2}, 3 \omega_{1}$, and $3 \omega_{2}$ ) and the 2nd order intermodulation products (at $\omega_{1}-\omega_{2}$ and $\omega_{1}+\omega_{2}$ ) are related far away from the fundamental frequency, compared to some $3 r d$ order intermodulation products (at $2 \omega_{1}-\omega_{2}$ and 
$\left.2 \omega_{2}-\omega_{1}\right)$. Therefore, usually odd orders of distortions are usually considered when modeling a PA, especially when the carrier frequency is usually from hundreds $\mathrm{MHz}$ to a few $\mathrm{GHz}$ in wireless communication systems.

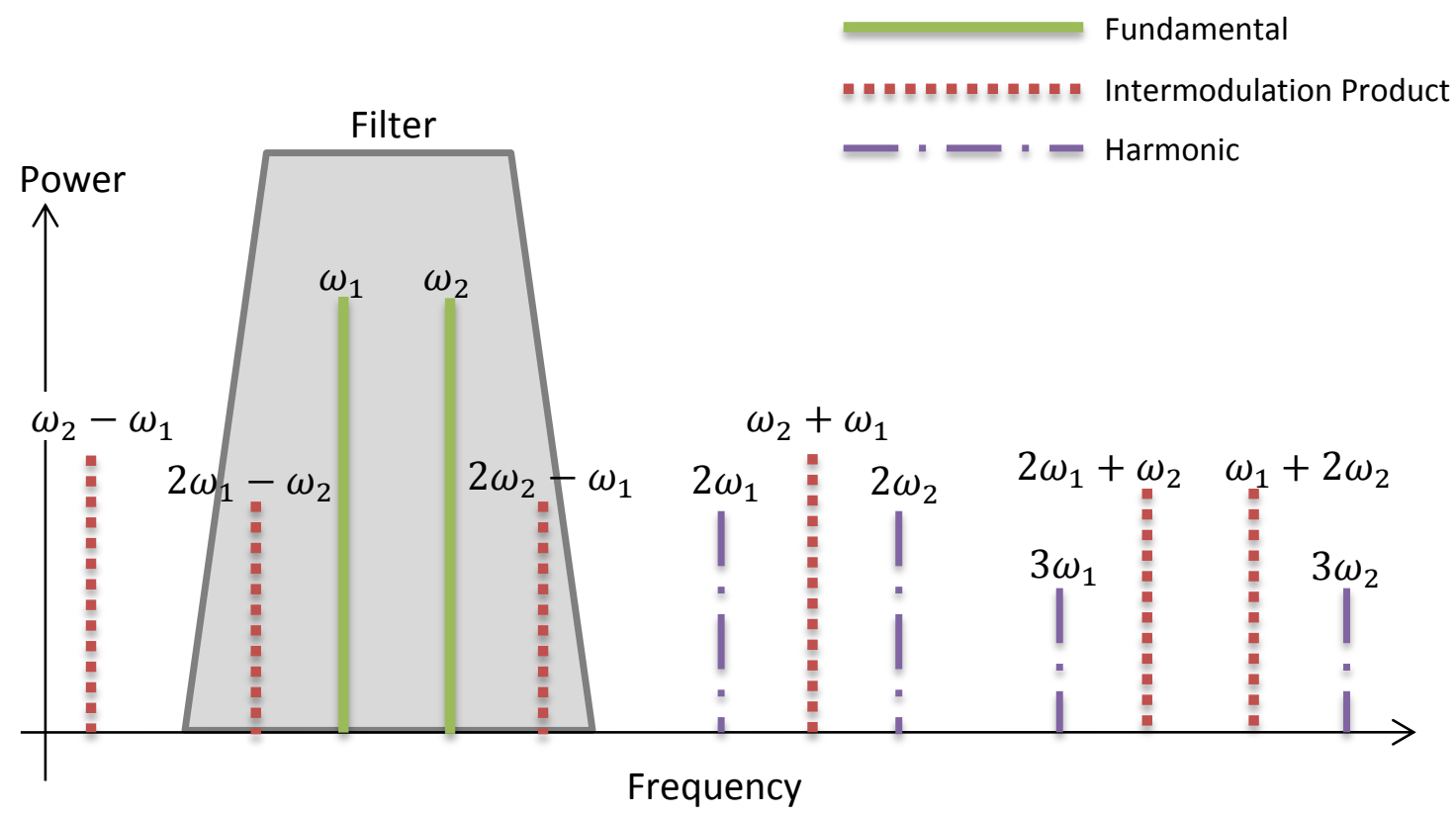

Figure 7 Frequency presentation of each distortion term

If the 3rd PA model is expanded to an arbitrarily high order $n$, the amplified signal is

$$
\begin{aligned}
v(t)= & a_{1} A\left(\cos \omega_{1} t+\cos \omega_{2} t\right)+a_{3} A^{3}\left(\cos \omega_{1} t+\cos \omega_{2} t\right)^{3}+\cdots \\
& +a_{n} A^{n}\left(\cos \omega_{1} t+\cos \omega_{2} t\right)^{n}
\end{aligned}
$$

For a band-pass systems, only the odd order intermodulation products at $\left(\frac{n-1}{2} \omega_{1}-\right.$ $\left.\frac{n+1}{2} \omega_{2}\right)$ and $\left(\frac{n+1}{2} \omega_{1}-\frac{n-1}{2} \omega_{2}\right)$ fall within the passband and cannot be filtered out, 
this causes distortion. If the components out of the pass-band are filtered out, we can rewrite (2.14) as

$$
\begin{aligned}
v(t)= & b_{1}\left(\cos \omega_{1} t+\cos \omega_{2} t\right)+b_{3}\left[\cos \left(\omega_{1}-2 \omega_{2}\right) t+\cos \left(2 \omega_{1}-\omega_{2}\right) t\right] \\
& +\cdots+b_{n}\left[\cos \left(\frac{n-1}{2} \omega_{1}-\frac{n+1}{2} \omega_{2}\right) t+\cos \left(\frac{n+1}{2} \omega_{1}-\frac{n-1}{2} \omega_{2}\right) t\right]
\end{aligned}
$$

where the coefficient $b_{n}$ of $\mathrm{n}$-th order intermodulation components, for odd $n$, is [34]

$$
b_{n}=\frac{1}{2^{n-1}}\left(\begin{array}{c}
n \\
\frac{n+1}{2}
\end{array}\right) a_{n} A^{n}
$$

where $\left(\begin{array}{l}p \\ q\end{array}\right)$ gives the number of different combinations of q elements that can be chosen from an p-element set.

Figure 8 shows the definition of the $\mathrm{n}$-th order intercept point $I P_{n}$, defined as the point where the output power at the fundamental frequency $P_{o}$ at $\omega_{1}$ and the power of $\mathrm{n}$-th order intermodulation response $P_{\left(\frac{n-1}{2} \omega_{1}-\frac{n+1}{2} \omega_{2}\right)}$ intersect. The plot of each on a log-log scale is a straight line with a slope corresponding to the order of the response, i.e., the response at $\omega_{1}$ will have a slope $1: 1$ and the response at $\left(\frac{n-1}{2} \omega_{1}-\frac{n+1}{2} \omega_{2}\right)$ will have a slope $\mathrm{n}: 1$. Their intersection $K$ is the $\mathrm{n}$-th order intercept point. 


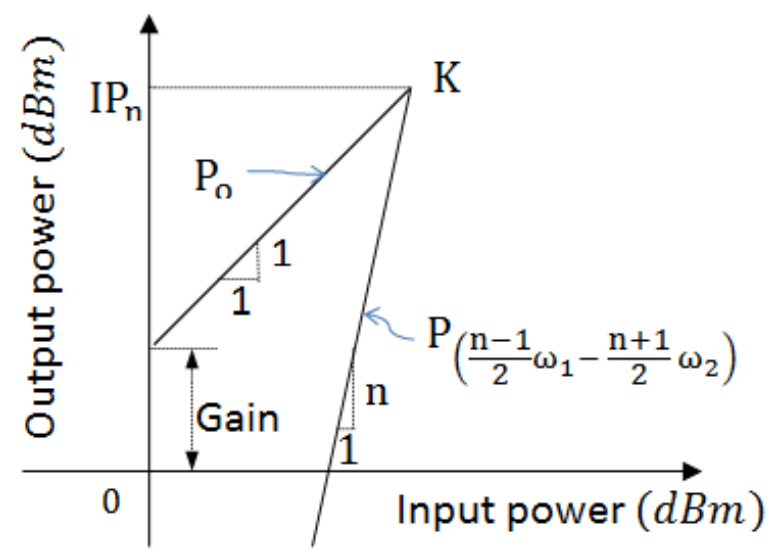

Figure 8 The definition of the $n$-th order intercept point

\subsubsection{The $1 \mathrm{~dB}$ Compression Point}

Most nonlinear devices, such as PAs or mixers, tend to become lossier with increasing input power. At a certain power level, the gain response of the device is reduced by a specific amount. This power level is said to be the compression point. The $1 d B$ compression point $\left(P_{1 d B}\right)$ indicates the power level that causes the gain to drop by $1 \mathrm{~dB}$ from its small signal value. The definition is showed in Figure 9. The $1 d B$ compression point is the point at which signal distortion becomes a serious problem. For most cases, the input $I P_{3}$ is about $8 d B$ to $10 d B$ higher than the input $P_{1 d B}$. In a linear amplifier, such as class $A$, the gain on the input to output characteristic plot is a constant about $6 d B$ to $10 d B$ lower than the $P_{1 d B}$. 


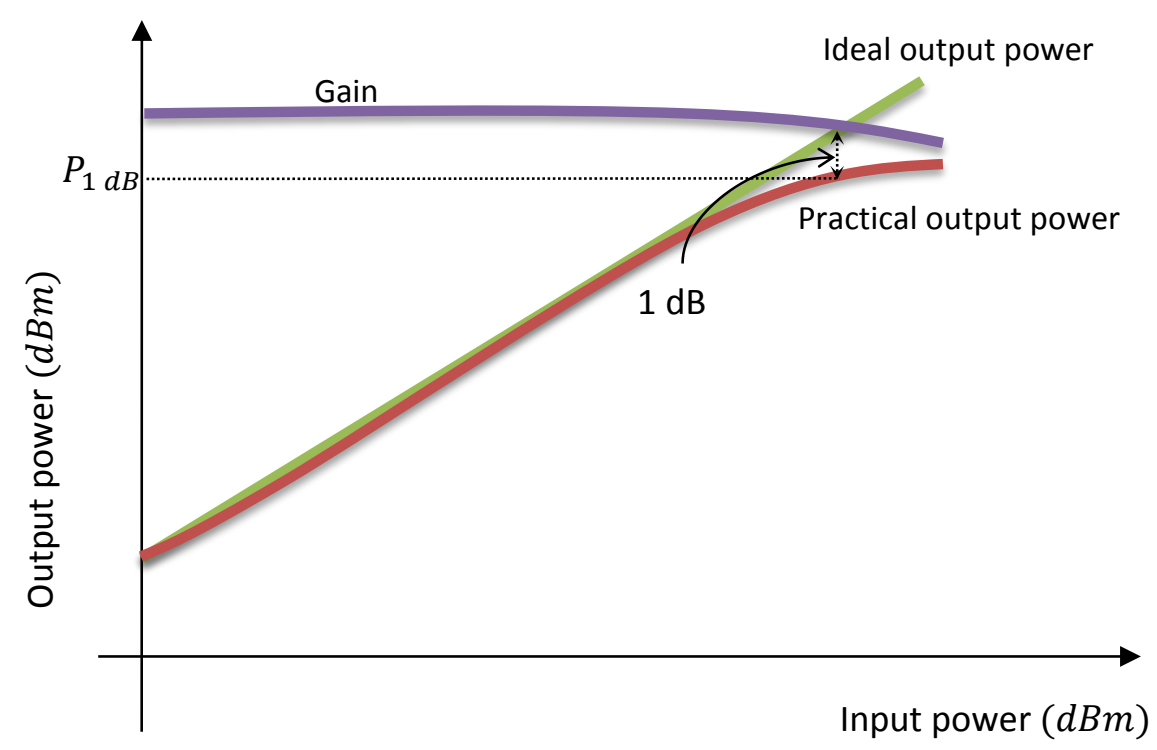

Figure 9 The definition of the $1 \mathrm{~dB}$ compression point

\subsection{Nonlinearity With Memory Effect}

Newer transmission formats are especially vulnerable to the nonlinear distortions due to their high peak-to-average power ratios (PAPRs). As the result, the nonlinearity analysis that only considers memoryless distortion is not accuracy enough anymore. Memory is caused by the stored energy to be charged or discharged. The most significant memory effect appears in Class $A B$ amplifiers, with reduced conduction angles where drain/collector current varies with output power. In Class A amplifiers, the memory effect reduces [28]. Memory effect could be electrical or thermal. Electrical effects are the dominant source of memory effects in wideband communication systems. The fundamental reason is the frequency-dependency of the bias and matching networks. The matching impedance network cannot guarantee perfect cancellation due 
to its static nature and the dynamic behavior in the PA is visible as hysteresis in the envelope transfer characteristics. .Careful design of the bias networks can reduce the electrical memory effects. The thermal memory effects are caused by electro-thermal couplings. [29].

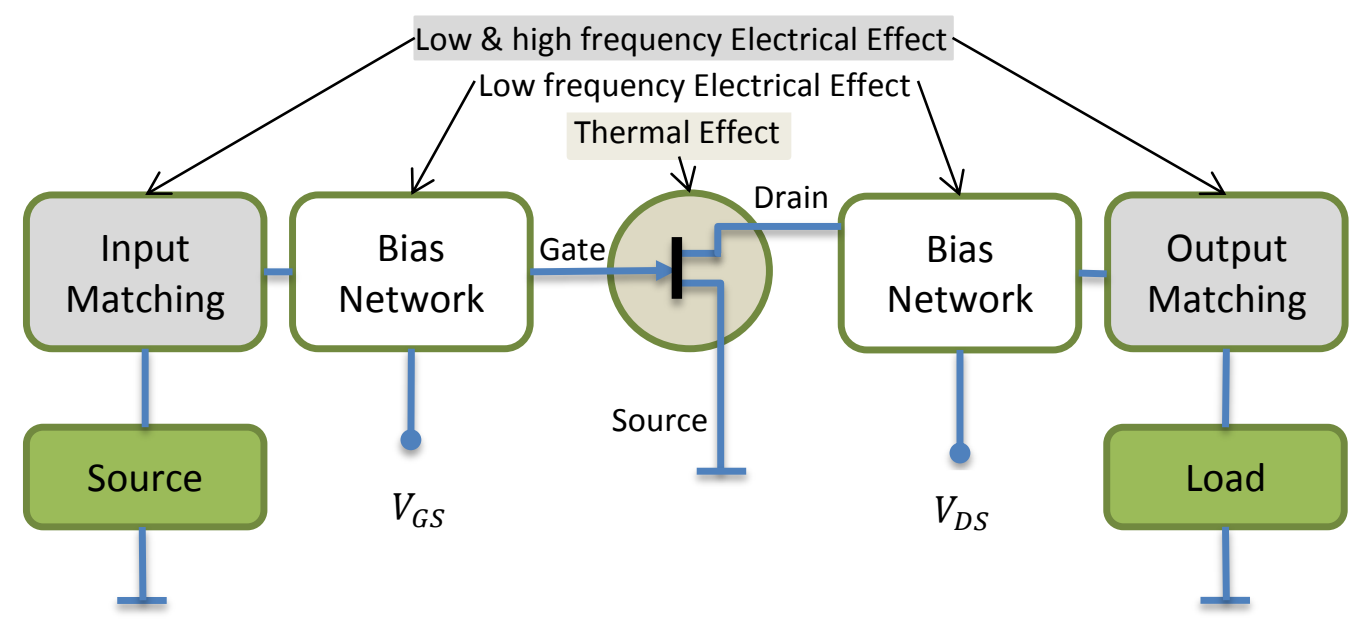

Figure 10 Typical source of memory effect

With memory effect, the output signal of an RF power amplifier usually shows a significant amount of asymmetry between the upper and lower intermodulation product as Figure 11. 


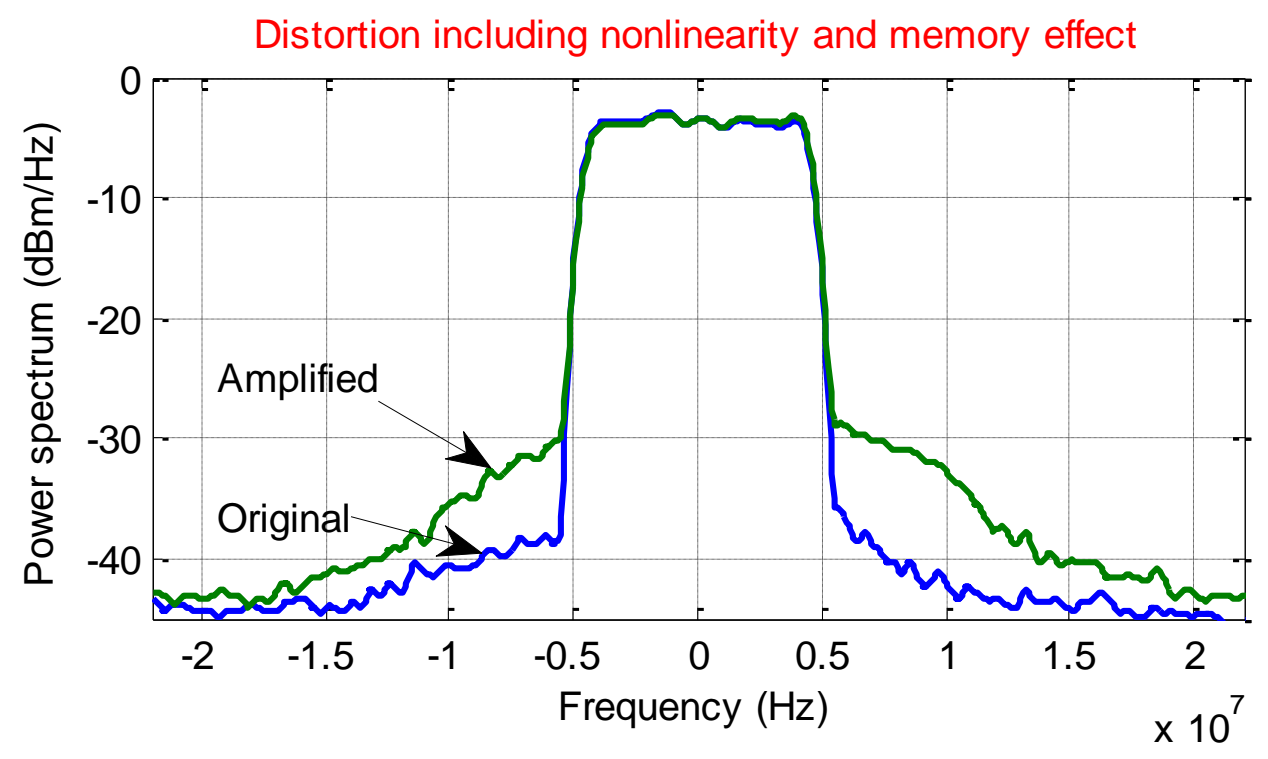

Figure 11 Spectrum regrowth with memory effect

It is known that the asymmetric spectrum come from memory effects, which may arise from thermal effect, and long-time constants in DC bias networks. Memory effect is a non-constant distortion behavior at different frequencies (mainly of envelope frequency) and thus distorts the symmetry of the power spectrum. Several researches have recently been conducted to deal with the asymmetric effect in PA nonlinearity: In [35] S . C. Cripps explained this asymmetric effect using an envelope domain phase shift that depends on the amplitude distortion and its interaction with the AM/AM and AM/PM functions. J. Vuolevi, T. Rahkonen et al. [36] suggested that the opposite phases of the thermal filter at the negative and positive envelope frequencies causes IMD power to add at one sideband, while subtracting at the other. N. B. Carvalho and J. C. Pedro [37] explained that the derived necessary condition for intermodulation product asymmetry generation was that the nonlinear device must see significant reactive 
baseband load impedance, provided the real part of the intermodulation product do not override the imaginary parts of baseband and second harmonic contributions.

High power amplifiers and/or wideband signals applied to a PA exhibit memory effects. Memoryless predistorter cannot reduce intermodulation products satisfactorily in high power amplifiers with wideband signals. The traditional memoryless AM/AM and AM/PM characteristics do not contain complete information about the nonlinearity, the accuracy of PA model is thus reduced.

For memoryless cases, a table of predistorter gain values can be stored for every possible input envelope value. If this table is applied to the PA input, then it should cancel the undesired PA nonlinear response. For systems with memory effect, the output depends on both the current and the past input values, and the AM/AM and AM/PM characteristics are dynamic. It is interesting to observe the blurring effects on the $A M / A M$ and $A M / P M$ curves. This is consequence of the memory effects of the PA under test shown in Figure 12 and Figure 13. Figure 14 shows the measured sensitivity to bandwidth AM/AM under a four-carrier WCDMA input signal shows more dispersion in comparison to that under a one-carrier WCDMA input signal. 


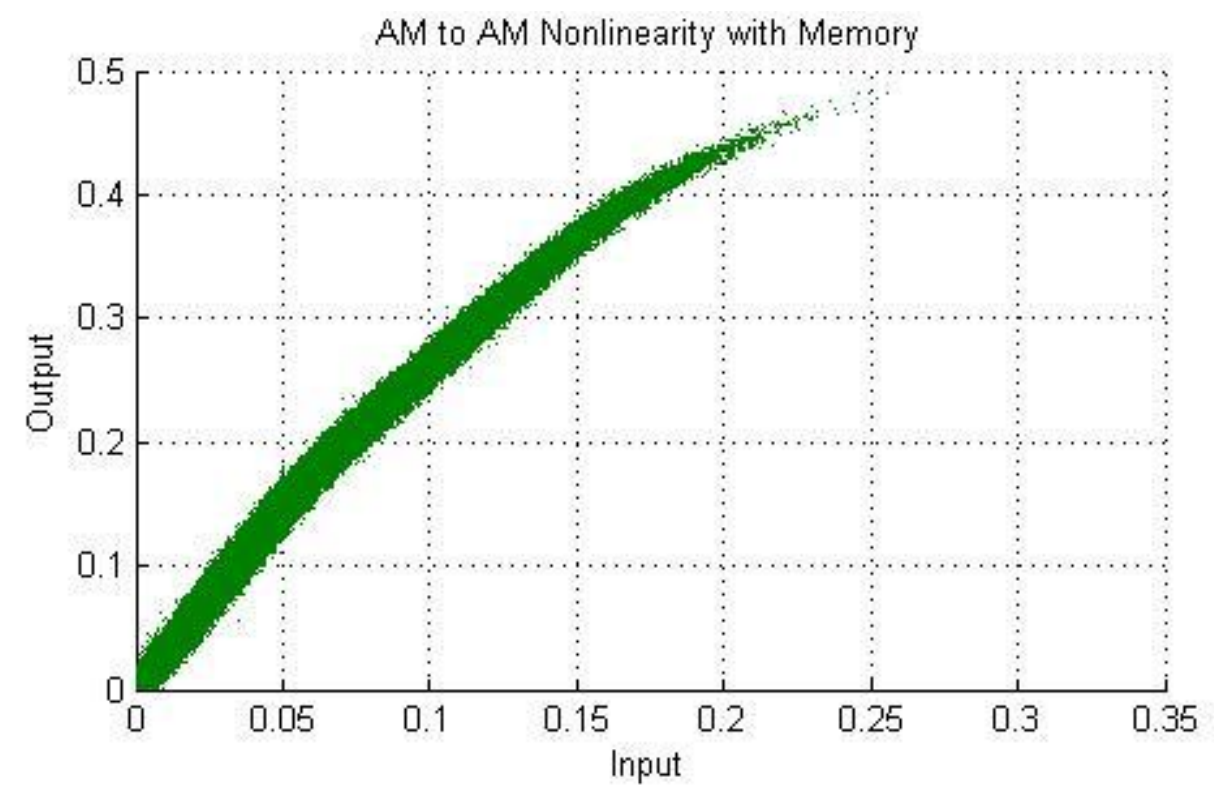

Figure $12 A M$ to $A M$ nonlinearity, scatter due tomemory effect

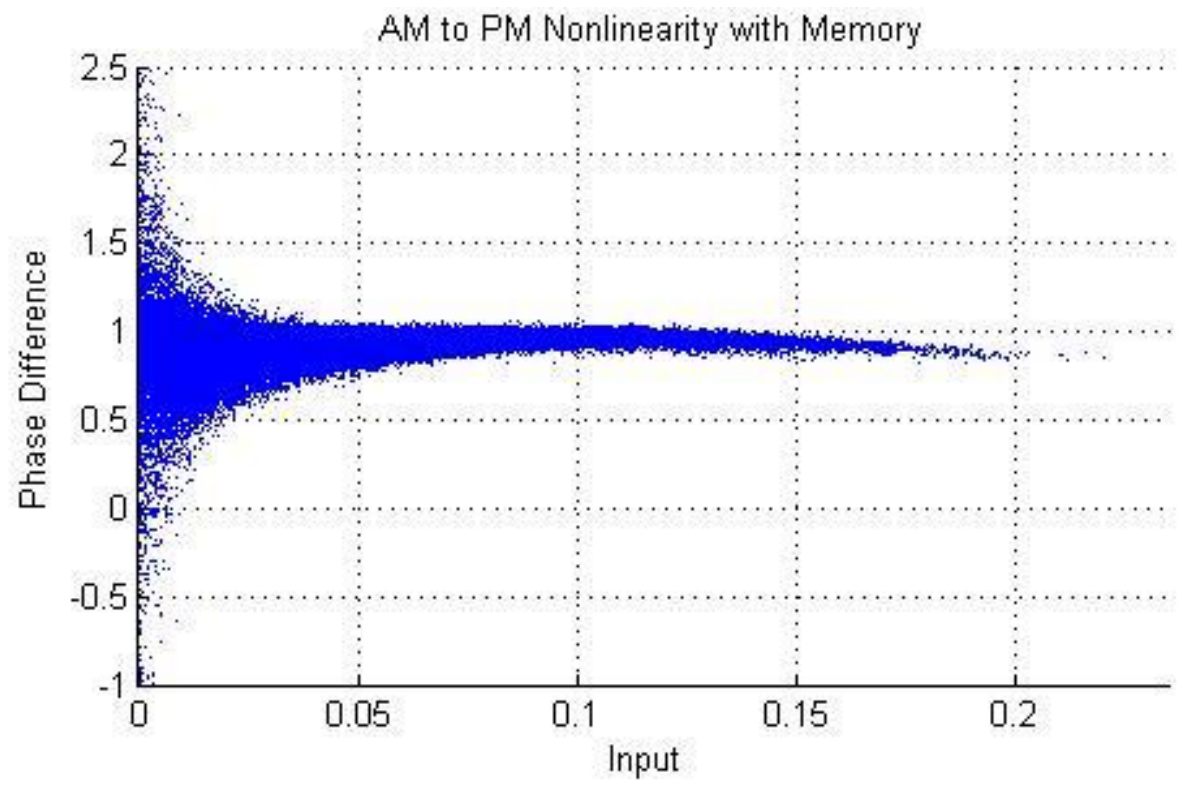

Figure 13 AM to PM nonlinearity, scatter due to memory effect 

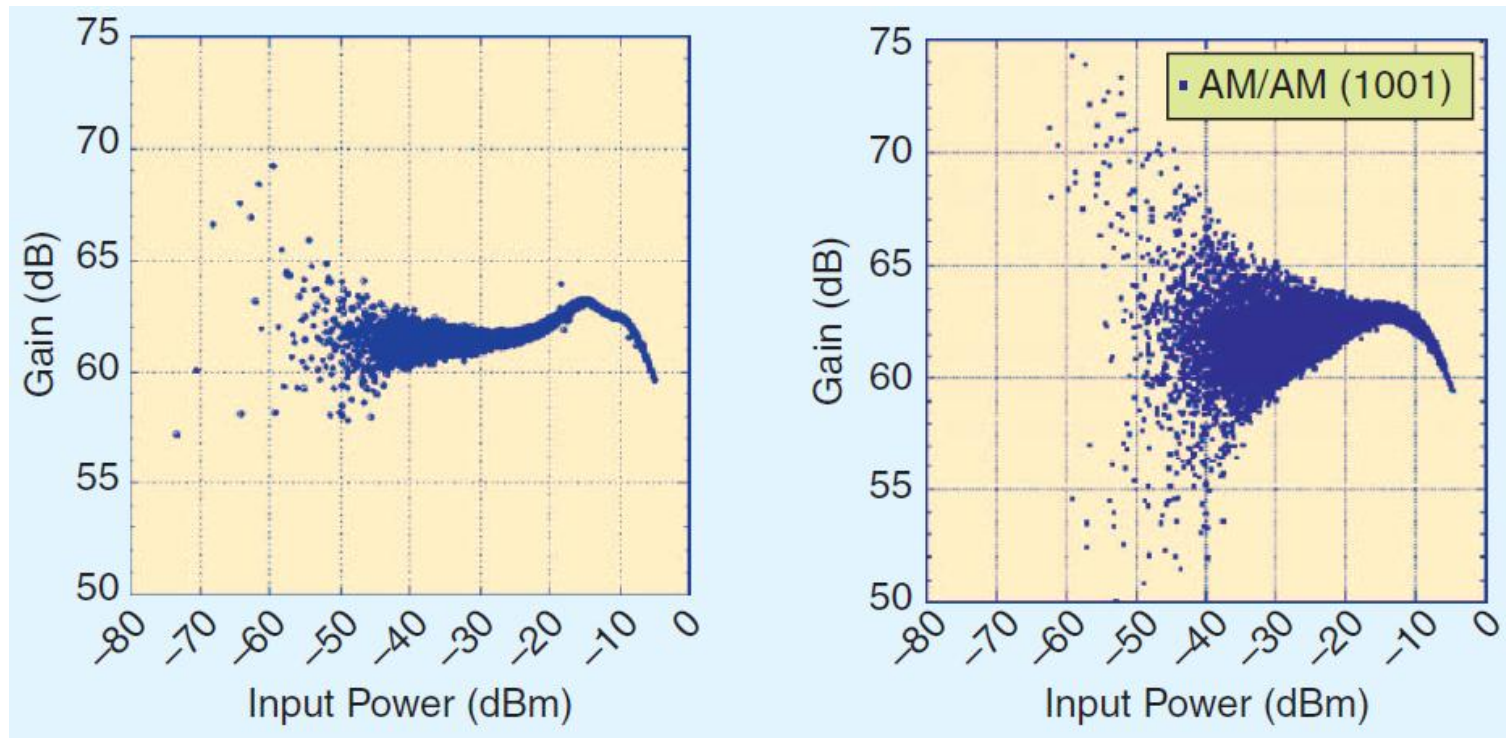

Figure 14 AM/AM under a one-carrier and a four-carrier WCDMA input signals, scatter due to [38]

Whatever the reason may cause the memory effects, a model to describe this phenomenon is needed for power amplifier analysis. If the asymmetric spectrum regrowth or scatter $\mathrm{AM} / \mathrm{AM}$ and $\mathrm{AM} / \mathrm{PM}$ distortion are not considered in predistortion design, the optimal intermodulation product reduction cannot be achieved for PA with asymmetries. This severely degrades predistortion performance.

In many applications involving modeling of nonlinear systems, it is convenient to employ a simpler model. The cascade connection of a linear time invariant (LTI) system and memoryless nonlinear system has been used to model the nonlinear PA with memory [29] [26] [39] [17] [40]. 
Chapter 3 Modeling and Spectrum Regrowth Analysis of Power amplifiers

\subsection{PA Modeling Review}

In general, power amplifiers can be modeled by several approaches: Physics-based approaches, circuit-based approaches, and black box-based (behavioral) approaches. The black box-based approach is most efficient, and is usually used in modeling the nonlinear distortion of PAs.

Black box-based PA modeling can be classified into three categories: Memoryless models, quasi-memoryless models, and models with memory effect [41]. The table 2 shows the detail characteristics of each category [41]. $f_{m}$ is the envelop frequency of input signals of power amplifiers.

\begin{tabular}{|c|c|c|c|}
\hline & $\begin{array}{c}\text { Characteristics of } \\
\text { Models }\end{array}$ & Typical methods & Notes \\
\hline $\begin{array}{c}\text { Memoryless } \\
\text { Models }\end{array}$ & AM/AM Only & Polynomials & $\begin{array}{c}\text { without Phase } \\
\text { Distortion }\end{array}$ \\
\hline $\begin{array}{c}\text { Quasi-memoryless } \\
\text { Models }\end{array}$ & AM/AM \& AM/PM & $\begin{array}{c}\text { Complex } \\
\text { Polynomials }\end{array}$ & $\begin{array}{c}\text { with Short Term } \\
\text { memory effect } \\
\text { (delay< }<\frac{1}{f_{m}} \text { ) }\end{array}$ \\
\hline $\begin{array}{c}\text { Models with } \\
\text { Memory Effect }\end{array}$ & $\begin{array}{c}\text { frequency } \\
\text { dependent }\end{array}$ & $\begin{array}{c}\text { Volterra, } \\
\text { Wiener, or } \\
\text { Hammerstein }\end{array}$ & $\begin{array}{c}\text { with Long term } \\
\text { Memory Effect }\end{array}$ \\
\hline
\end{tabular}

Table 2 The categories of PA models 


\subsubsection{Memoryless Model and Quasi-memoryless Model}

Both types of memoryless power amplifiers can be mathematically modeled by the wellknown Taylor series (Polynomial) as

$$
v(n)=a_{1} w(n)+a_{3} w^{3}(n)+\cdots+a_{M} w^{M}(n) .
$$

where $w(n)$ is input signal and $v(n)$ is the output signal of a PA in passband.

If the system is considered in baseband, the PA model can be expressed as

$$
\hat{v}(n)=\hat{a}_{1} w(n)+\hat{a}_{3} w^{3}(n)+\cdots+\hat{a}_{M} w^{M}(n) .
$$

where $\hat{a}_{n}=\frac{1}{2^{n-1}}\left(\begin{array}{c}n \\ \frac{n+1}{2}\end{array}\right) a_{n}$.

The advantage for using Taylor series model is that it provides a simple, yet effective way to describe the nonlinearity of PAs. The difference between memoryless and quasimemoryless models is the coefficients. The coefficients of a memoryless model are all real, and those of a quasi-memoryless model are complex. In a memoryless model, only the magnitude response is considered for nonlinearity analysis, but this is not enough when the phase distortion of PAs needs to be considered. The complex coefficients of the quasi-memoryless models include both $A M / A M$ and $A M / P M$ distortion, and are often used by designers and researchers when weak memory effect is shown in PAs. 
The advantage of a memoryless model is that the coefficients could be calculated from the intercept points of the PAs directly, the spectrum regrowth could be predicted only using only those intercept points. This is helpful for the component engineers in the design and testing of RF power amplifiers when a communication standard specifies the in-band and out-of-band emission level [42] [8] [10] [43]. However, for a quasimemoryless model, the coefficients have to be extracted from measurement.

\subsubsection{Models with Memory Effect}

If an attempt is made to amplify wideband signals, where the bandwidth of the signal is comparable with the inherent bandwidth of the amplifier, frequency-dependent behaviour will be encountered in the system. Even a quasi-memoryless model cannot describe the PA completely. Therefore, models with memory effect are proposed. These models are categorised as two-box, three-box or parallel-cascade models [39]. The twobox methods are the most typical and the most frequently used to model the nonlinearity with memory effect.

As such, the Volterra series have been applied for PA modelling, in discrete time domain, as [44]

$$
v(n)=\sum_{m=0}^{M} a_{m}\left(l_{1}, l_{2}, \ldots, l_{m}\right) \prod_{i=1}^{m} w\left(n-l_{i}\right)
$$

It can be seen that the number of coefficients of the Volterra series increases 
exponentially as the memory length and the nonlinear order increase. This drawback makes the Volterra series unattractive for real-time applications [17]. Therefore, some special cases of Volterra series are considered, such as the Wiener model [18], the Hammerstein model [19], and the memory polynomial model [20].

The Wiener model is a linear time-invariant (LTI) system followed by a memoryless nonlinearity system (see Figure 15). The two subsystems are given by

$$
\begin{aligned}
& v(n)=\sum_{l=0}^{L-1} a_{l} w(n-l), \\
& y(n)=\sum_{\substack{m=1 \\
\text { modd }}}^{M} b_{m} v^{m}(n),
\end{aligned}
$$

where $a_{l}$ are the coefficients (or impulse response value) of the LTI subsystem, and $b_{m}$ are the coefficients of the polynomial model presenting the nonlinearity. $L$ presents the maximum depth of delays caused by memory effect, and $M$ is the maximum order used in the model.

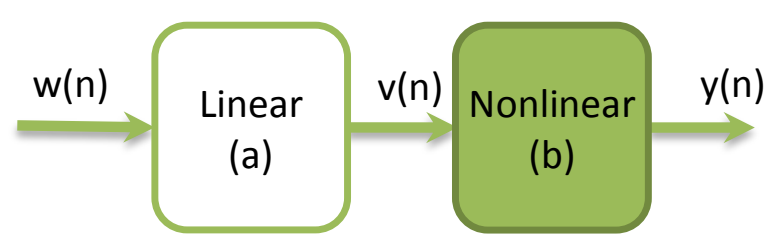

Figure 15 The Wiener model 
The Hammerstein model is another structure for modelling the nonlinear system with memory. Its only difference from the Wiener model is that it uses a memoryless nonlinearity system followed by an LTI system (see Figure 16). The two subsystems in this model are described by

$$
\begin{aligned}
& v(n)=\sum_{\substack{m=1 \\
m \text { odd }}}^{M} a_{m} w^{m}(n), \\
& y(n)=\sum_{l=0}^{L-1} b_{l} v(n-l),
\end{aligned}
$$

where $a_{m}$ are the coefficients of the polynomial model presenting the nonlinearity, and $b_{l}$ are the coefficients (or impulse response value) of the LTI subsystem.

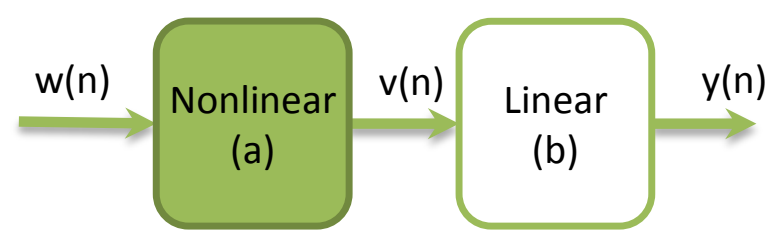

Figure 16 The Hammerstein model

By adding another LTI or nonlinearity block, or other similar structures improved the performance of the model, but the trade-off is the increased complexity of the systems and needs to identify multiple coefficients. Figure 17 and Figure 18 show the WienerHammerstein model and Hammerstein - Wiener model. The system identification toolbox of MATLAB supports the identification and analysis of the Hammerstein Wiener model. 


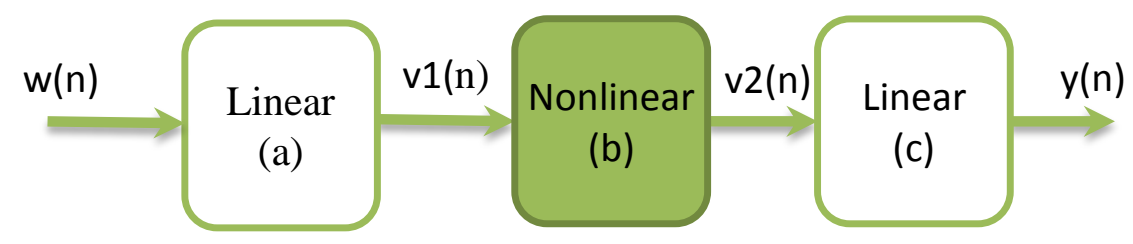

Figure 17 The Wiener-Hammerstein model

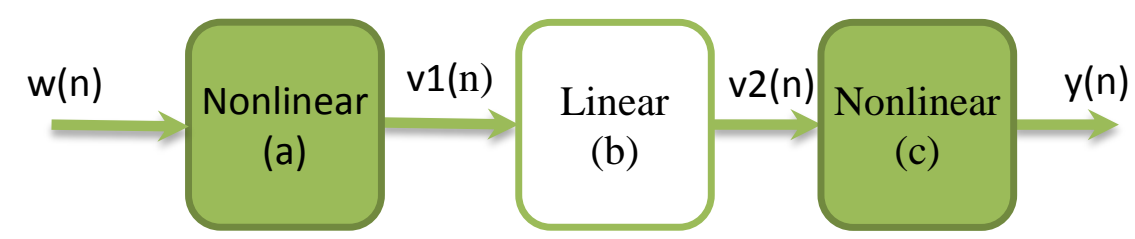

Figure 18 The Hammerstein-Wiener model

The parameter estimation of the above models is a classical Hammerstein or Wiener system identification problem. If no additional assumptions are made on the system's input signal $w(n)$, iterative Newton and Narendra-Gallman algorithms are the two most popular iterative estimation methods [40]. [45] provided a newer method by using two stage least-squares/singular value decomposition algorithm. However, the major disadvantage of these models is their excessive computational requirements leading to increased chip area and/or excessive power consumption. 
The memory polynomial model [20] using the diagonal kernels of the Volterra series is widely used since its parameters can be easily estimated by way of least-squares criteria.

$$
y(n)=\sum_{l=0}^{L-1} \sum_{\substack{m=1 \\ m \text { odd }}}^{M} a_{m, l} w^{m}(n-l),
$$

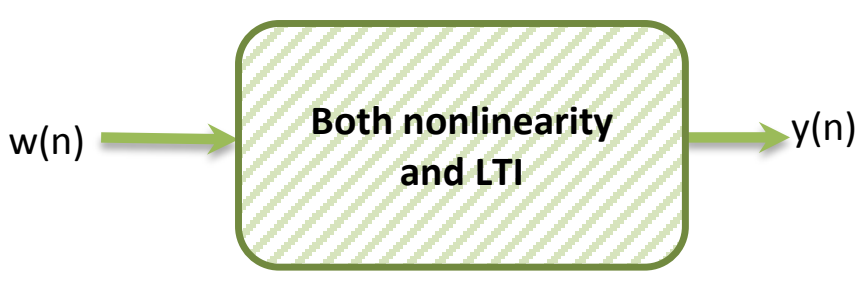

Figure 19 The memory polynomial model

However, this model cannot address the memoryless nonlinearity and the memory effect of a PA separately, so the complexity of the system is significant when PA shows little memory effect. For most applications, if the intermodulation products are delayed by the same time, the coefficient matrix of the whole PA model is 1 , so it is not necessary to use this model.

\subsection{Proposed PA Modeling Method}

Hammerstein model is used to model PAs with memory. A static subsystem describes the memoryless nonlinearity, and a dynamic subsystem followed to present memory 
effect shown in Figure 16. Parameter estimation of the PA model in Figure 16 is a classical Hammerstein system identification problem. If no additional assumptions are made on the system's input signal $w(n)$, iterative Newton and Narendra-Gallman algorithms are the two most popular iterative estimation methods [40]. However, since we can obtain the parameters of Block A from the characteristics of the amplifier, the only work remains is the identification of the LTI system.

\subsubsection{The Nonlinearity Part of Model}

The nonlinearity part of the proposed model is

$$
v(n)=A[w(n)]=\sum_{\substack{m=1 \\ m \text { odd }}}^{M} a_{m} w^{m}(n),
$$

where $w(n)$ is the input signal of PAs, and $v(n)$ is the output of the memoryless nonlinear block (a). Only the odd-order terms are considered. $A$ denotes the memoryless nonlinearity of PA, and $a^{\prime}$ s are the polynomial coefficients, which could be identified directly from the intercept points of PAs.

Traditionally, the nonlinearity of an RF amplifier is described by the 3rd order intercept point $\left(I P_{3}\right)$ usually given in the manufacturer's datasheets. For a PA with gain compression, the output using polynomial PA model can be expressed as a function of the gain, intercept points, and the input signal. The higher order intercept points are not generally provided, but they can easily be calculated from the higher order 
intermodulation products $(I M P)$ by using a two-tone test [33]. However, there is no generic mathematical relationship derived between the coefficients of polynomial model and higher order intercept points.

The relationship of the 5th order can be seen in our earlier published work, such as [11]. In [46], we generalized this relationship by expanding it up to an arbitrarily high n-th order, such that the nonlinear polynomial coefficient for any orders can be obtained from the intercept points of RF power amplifiers. This would help RF amplifier designers to model PA more precisely. Furthermore, the PA polynomial in Block (a) can be modeled directly by using $n$-th order intermodulation products from a simple two-tone test.

\subsubsection{Obtain Polynomial Coefficients from the Intercept Points of RF Power}

\section{Amplifiers}

From Chapter 2, we know the output power at fundamental frequency $P_{o}$ at $\omega_{1}$ and the power of $n$-th order intermodulation response $P_{\left(\frac{n-1}{2} \omega_{1}-\frac{n+1}{2} \omega_{2}\right)}$ intersect at the intercept point $I P_{n}$. Assume that the input and output impedance of the two-port are $Z_{i n}=$ $Z_{\text {out }}=R \Omega$, the output power at fundamental frequency is

$$
P_{o}=10 \log \left[\left(\frac{a_{1} A}{\sqrt{2}}\right)^{2} \frac{10^{3}}{R}\right] d B m
$$

Then, the power of $\mathrm{n}$-th order intermodulation product is given as 


$$
\begin{aligned}
P_{\left(\frac{n-1}{2} \omega_{1}-\frac{n+1}{2} \omega_{2}\right)} & =10 \log \left[\left(\frac{b_{n}}{\sqrt{2}}\right)^{2} \frac{10^{3}}{R}\right] \mathrm{dBm} \\
& =10 \log \left\{\left[\frac{\frac{1}{2^{n-1}}\left(\begin{array}{c}
n \\
\frac{n+1}{2}
\end{array}\right) a_{n} A^{n}}{\sqrt{2}}\right]^{2} \frac{10^{3}}{R}\right\} d B m .
\end{aligned}
$$

Since at intercept point $K$ by definition, $P_{o}=P_{\left(\frac{n-1}{2} \omega_{1}-\frac{n+1}{2} \omega_{2}\right)}$, by comparing (3.10) and (3.11) we obtain the theoretical amplitude $A$ at point $K$ as

$$
A^{(n-1)}=\frac{2^{(n-1)}}{\left(\begin{array}{c}
n \\
\frac{n+1}{2}
\end{array}\right)} \times \frac{a_{1}}{\left|a_{n}\right|} .
$$

Therefore, we have $I P_{n}=K$ when $R=1$. After changing $I P_{n}$ scale from $d B m$ to $d B W$,

$$
\begin{aligned}
& I P_{n}=10 \log \left(\frac{a_{1}{ }^{2} A^{2}}{2}\right) d B=\frac{2}{n-1} 10 \log \left[\frac{a_{1}{ }^{n-1} A^{n-1}}{2^{\frac{n-1}{2}}}\right] d B \\
& =\frac{2}{n-1} 10 \log \left[\frac{2^{\frac{n-1}{2}} a_{1}^{n}}{\left(\begin{array}{c}
n \\
\frac{n+1}{2}
\end{array}\right)\left|a_{n}\right|}\right] d B
\end{aligned}
$$

Furthermore, $a_{n}$ can be calculated reversely from (3.13). In general, we choose $a_{n}<0$, i.e. gain compression, and $a_{1}=10^{\frac{G}{20}}$, so the nonlinear model coefficients $a_{n}$ can be obtained from the intercept points of RF Power Amplifiers: 


$$
a_{n}=-\frac{2^{\frac{n-1}{2}}}{\left(\begin{array}{c}
n \\
\frac{n+1}{2}
\end{array}\right)} 10^{\left(\frac{-(n-1) I P_{n}+n G}{20}\right)}=-\frac{2^{\frac{n-1}{2}}}{\left(\begin{array}{c}
n \\
\frac{n+1}{2}
\end{array}\right)} 10^{\left(\frac{I M P_{n}-n P_{t}+n G}{20}\right)} .
$$

Therefore, the coefficients of polynomial PA model (Block a) can be acquired directly from a two-tone test in frequency domain.

\subsubsection{Verification of the Nonlinearity Coefficient Identification}

To verify the result in (3.14) and to illustrate the proposed modeling method, we set up the measurement instruments consisted of an Agilent E4438C vector signal generator and an Agilent 89600 vector signal analyzer. The PA we used is Mini-Circuits ZFL$1000 \mathrm{LN}+$ with a $13 \mathrm{dBm} \mathrm{IP}$ and a $20 \mathrm{~dB}$ gain.

Figure 20 shows the results of the two-tone test. The center frequency was $1 \mathrm{GHz}$, and two-tone spacing was $10 \mathrm{kHz}$. The coefficients of Taylor series model for the PA up to the 7th order in (3.9) were derived by (3.14). With these conditions, the modeled power spectrum matched the measured spectrum very well as shown in Figure 20. 


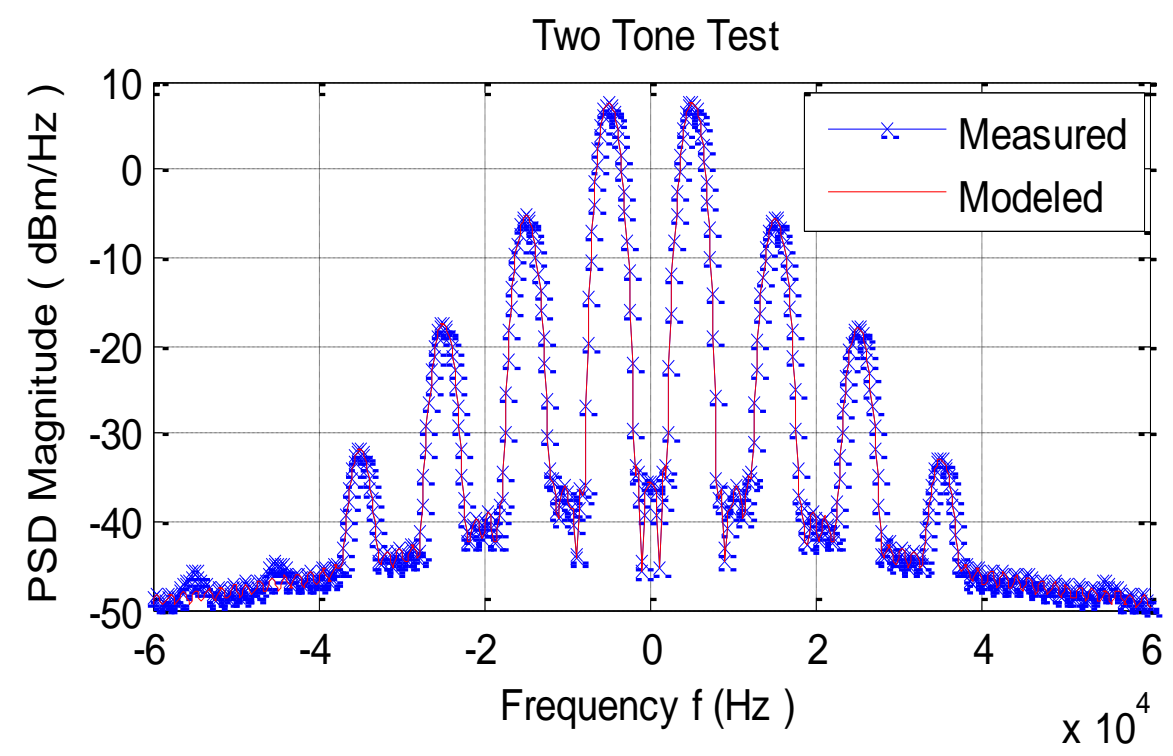

Figure 20 Amplified two-tone

Moreover, a 1.4 MHz bandwidth downlink 3GPP LTE Advanced signal was used here for further experimental verification. Figure 21 is the comparison of the modeled spectrum and the measured spectrum. It was observed that the PA model using only up to 3rd order or 5th order could not fully predict the nonlinearity, and the one with higher order (up to 7th order) fit the measurement much better. Also, higher order intermodulation terms occupied more bandwidth, so it was necessary to take higher orders intercept points into nonlinear analysis when the distorted spectrum occupied much broader bandwidth as seen in Figure 21. Therefore, it was necessary to include more orders of nonlinear elements for precise analysis. 


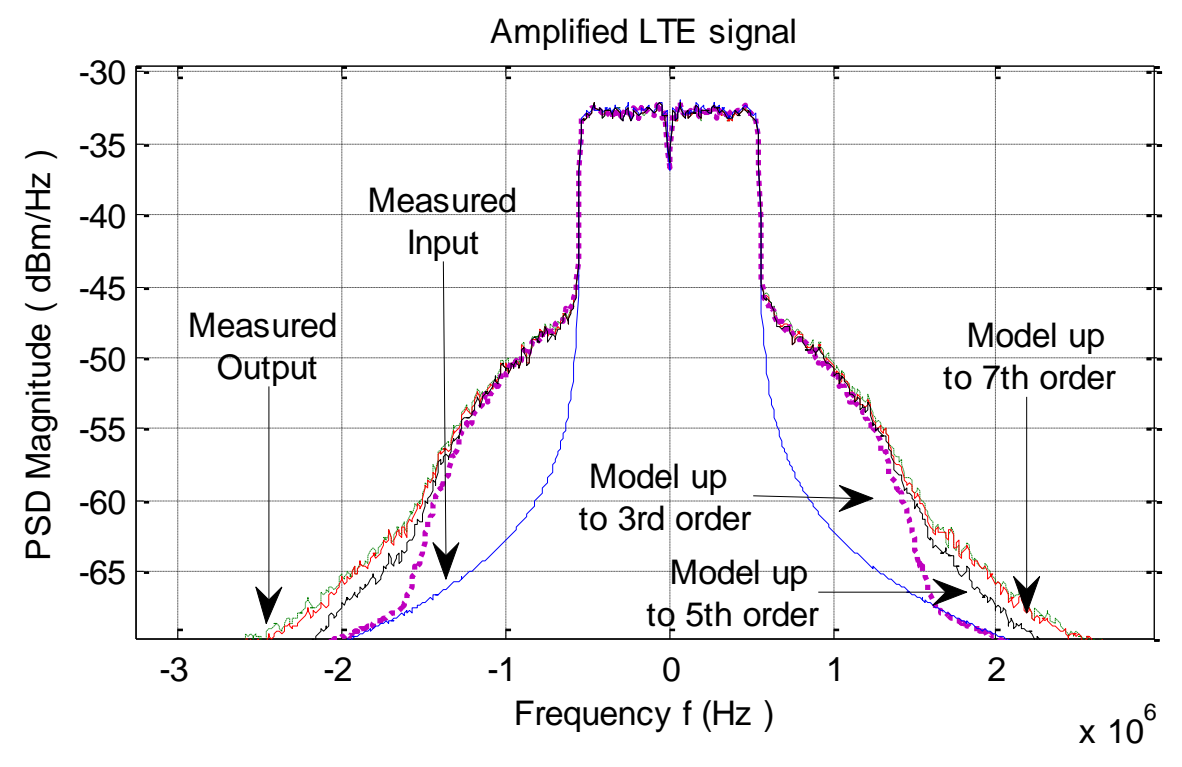

Figure 21 Amplified 1.4 MHz LTE downlink signal

With this result, the memoryless polynomial of each RF power amplifier can be determined from its intercept points or intermodulation products completely. This method could be used for designing power amplifiers or predistorters.

Figure 22 plots the power spectrum of each individual term in (3.9) up to the 7 order, and the sum is the same as the one using 7 order model in Figure 21. Higher order intermodulation terms occupy more bandwidth, which means it is necessary to take higher order intercept points into nonlinear analysis when the distorted spectrum occupied much broader bandwidth as seen in Figure 21. 


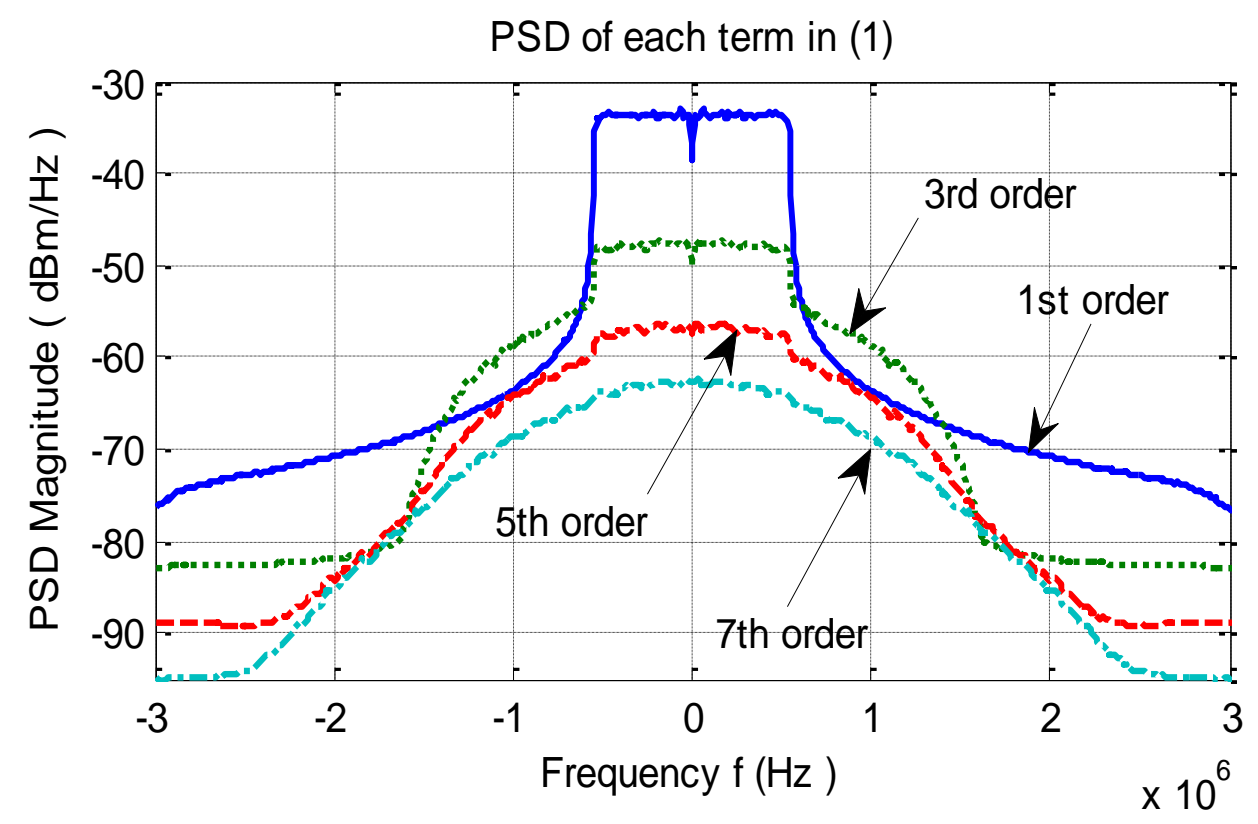

Figure 22 Spectrum of the Intermodulation product of each individual term in (3.9)

\subsubsection{The Memory Effect Part of Model}

For memory effect block (b), the output depends on both current and past input values, therefore time delay of both input and output signals need to be considered. Autoregressive Moving-Average (ARMA) model is used to present this causal, linear, time invariant (LTI) subsystem as

$$
\sum_{k=0}^{K} c(k) y(n-k)=\sum_{l=0}^{L} b(l) v(n-l)
$$

with $y(n)$ and $v(n)$ being the output and input functions of LTI subsystem, where $k$ and $l$ are the delays of input and output signal respectively, contributing to the description of the memory effect. In general, the delay is assumed to be the same in most cases. $L$ 
and $\mathrm{K}$ present the memory depths. $c(k)$ and $b(l)$ are the coefficients of AR and MA parts of the ARMA model. The transfer function of this ARMA model is:

$$
H(z)=\frac{\sum_{k=0}^{K} c(k) z^{-k}}{\sum_{l=0}^{L} b(l) z^{-l}}
$$

The advantages of ARMA filter are: The coefficients can be easily obtained from several mature algorithms, and the memory effect can be reduced easily by using a predistortion structure that has inverse transfer function of the ARMA filter. A prior stability test maybe needed due to the instabilities of the IIR filter.

For coefficient identification of an ARMA model where the model order is not known, selecting the model order is a key first step for estimating the model parameters. Several information theoretic criteria have been proposed for this model order selection task. Based on the maximum likelihood principle, the Akaike information criterion (AIC) [47] is the first popular automatic method for order selection for ARMA time series. Using the AIC, one selects the order $(p, q)$ which minimizes a likelihood-related term $\ln \widehat{\sigma}_{\mathrm{p}, \mathrm{q}}^{2}$ plus a penalty term for order size:

$$
\operatorname{AIC}(p, q)=\ln \hat{\sigma}_{p, q}^{2}+2(p+q)
$$

where $\widehat{\sigma}_{\mathrm{p}, \mathrm{q}}^{2}$ is the maximum likelihood. Some other improvements based on AIC criterion such as the minimum description length (MDL) [47] and minimum eigenvalue (MEV) criterion [48] can also be used for order selection of ARMA models. 
The second challenge for determining model coefficients is the estimation of the model coefficients. Some of the existing methods include Prony, Pade, Least Square, Shank, Autocorrelation, and Autocovariance methods [49]. In MATLAB, armax is used. An iterative search algorithm with the properties 'SearchMethod', 'Maxlter', 'Tolerance', and 'Advanced' minimizes a robustified quadratic prediction error criterion. The iterations are terminated either when Maxlter is reached, or when the expected improvement is less than Tolerance, or when a lower value of the criterion cannot be found.

The experimental verification of the proposed model is analyzed in Chapter 4. 


\section{Chapter 4 Spectrum Regrowth Analysis of 4G Communication Signals}

Previously, Dr. Heng Xiao, a member of the research team led by Dr. Fu Li, has analyzed the memoryless spectrum regrowth of an RF power amplifier in CDMA (IS-95 standard), MIR, and GSM systems in collaboration with Dr. Qiang Wu from a nearby industry. He developed expressions for out-of-band emission levels of the signals in these systems in terms of the power amplifier's intermodulation coefficients and the power level and bandwidth [8] [11]. Dr. Chunming Liu developed expressions for TDMA (IS-54 standard), Motorola iDEN and Wi-Fi systems [9] [42] [7]. I have analyzed the spectrum regrowth of TD-SCDMA signals in my master thesis [10]. This technology has been advanced further to 4 G communication signals (LTE and WiMAX) and the validity of the theoretical results derived by real experiments was verified [43].

LTE and WiMAX are two emerging wireless technologies which provide high speed mobile data and telecommunication services. Both of them support frequency division duplexing (FDD) and time-division duplexing (TDD) modes, and have more deployment flexibility than previous $3 G$ systems by using scalable channel bandwidths with different numbers of subcarriers while keeping frequency spacing between subcarriers constant.

Orthogonal Frequency Division Multiplexing (OFDM) with cyclic prefix (CP), rather than signal carrier modulation schemes in the traditional cellar systems, is used in downlink of LTE systems and both uplink and downlink of WiMAX systems. These two standards 
have set the specific requirements in terms of power spectrum density (PSD) of the signals for the controlling in-band and out-of-band spectrum re-growth. As the result, it is very important to know the relationship between the spectrum regrowth and intermodulation parameters of the system power amplifier.

\subsection{The Equivalent Mathematical Model of LTE and WiMAX}

The mathematical model of the transmitted OFDM signal $s(t)$ can be presented as [43]

$$
\begin{aligned}
s(t)= & \operatorname{Re}\left\{\sum _ { l = - \infty } ^ { \infty } w _ { T _ { s y m b o l } } ( t - l T _ { S } ) \left[\sum_{k=-\frac{N_{S c}}{2}}^{-1} a_{k, l} \cdot e^{j 2 \pi \Delta f\left(t-T_{C P}-l T_{s}\right)}\right.\right. \\
& \left.\left.+\sum_{k=1}^{\frac{N_{s c}}{2}} a_{k, l} \cdot e^{j 2 \pi \Delta f\left(t-T_{C P}-l T_{s}\right)}\right] e^{j 2 \pi f_{c} t}\right\}
\end{aligned}
$$

where $\operatorname{Re}\{\cdot\}$ denotes the real part of $\{\cdot\}$.

An OFDM symbol is constructed as an inverse Fourier transform (IFFT) of a set of $a_{k, l}$, which is the modulated transmitted data in the $l$ th OFDM symbol and the $k$ th subcarrier. To make the spectrum decrease more rapidly, a time window $w_{T_{\text {symbol }}}$ is applied to the individual OFDM symbols. $N_{s c}$ is the number of used subcarriers. $\Delta f$ is the subcarrier frequency spacing. $T_{C P}$ is a guard interval time to create the "circular prefix" to avoid the intersymbol interference (ISI) from the previous symbol, and $T_{S}$ is sample period. 
Taking $10 \mathrm{MHz}$ data rate transmission for example, the parameters used in math model for LTE and WiMAX signals are listed in Table 3.

\begin{tabular}{|c|c|c|}
\hline OFDM parameters & LTE & WiMAX \\
\hline Subcarrier frequency spacing, $\Delta \mathrm{f}$ & $15 \mathrm{kHz}$ & $10.9375 \mathrm{kHz}$ \\
\hline Number of used subcarriers, $\mathrm{N}_{\mathrm{Sc}}$ & 600 & 865 \\
\hline FFT size & 1024 & 1024 \\
\hline Useful symbol time $\mathrm{T}_{\text {symbol }}$ & $66.67 \mu \mathrm{s}$ & $91.43 \mu \mathrm{s}$ \\
\hline Data modulation & QPSK, $16-\mathrm{QAM}$, or $64-$ & BPSK, QPSK, $16-$ \\
& QAM & QAM, or $64-\mathrm{QAM}$ \\
\hline Sample frequency, $1 / \mathrm{T}_{\mathrm{S}}$ & $15.36 \mathrm{MHz}$ & $11.2 \mathrm{MHz}$ \\
\hline circular prefix, $\mathrm{T}_{\mathrm{CP}}$ & $4.69 \mu \mathrm{s}$ & $11.43 \mu \mathrm{s}$ \\
\hline
\end{tabular}

Table 3 The parameters used in math model for $10 \mathrm{MHz}$ data rate

The general expression for PSD of an OFDM baseband signal can be obtained as:

$$
P_{r}(f)=R_{s}\left\{\sum_{k=-\frac{N_{s c}}{2}}^{-1}\left|W_{T_{\text {symbol }}}(f-k \Delta f)\right|^{2}+\sum_{k=1}^{\frac{N_{S c}}{2}}\left|W_{T_{\text {symbol }}}(f-k \Delta f)\right|^{2}\right\}
$$

where $R_{s}=1 / T_{S}$ is the symbol rate and $W_{T_{\text {symbol }}}(f)$ is the Fourier Transform of the time-window function $w_{T_{\text {symbol }}}(t)$. Figure 23 shows the comparisons of LTE and WiMAX signals obtained through mathematical prediction, simulation in MATLAB and measurement with Vector Signal Generator, respectively. 

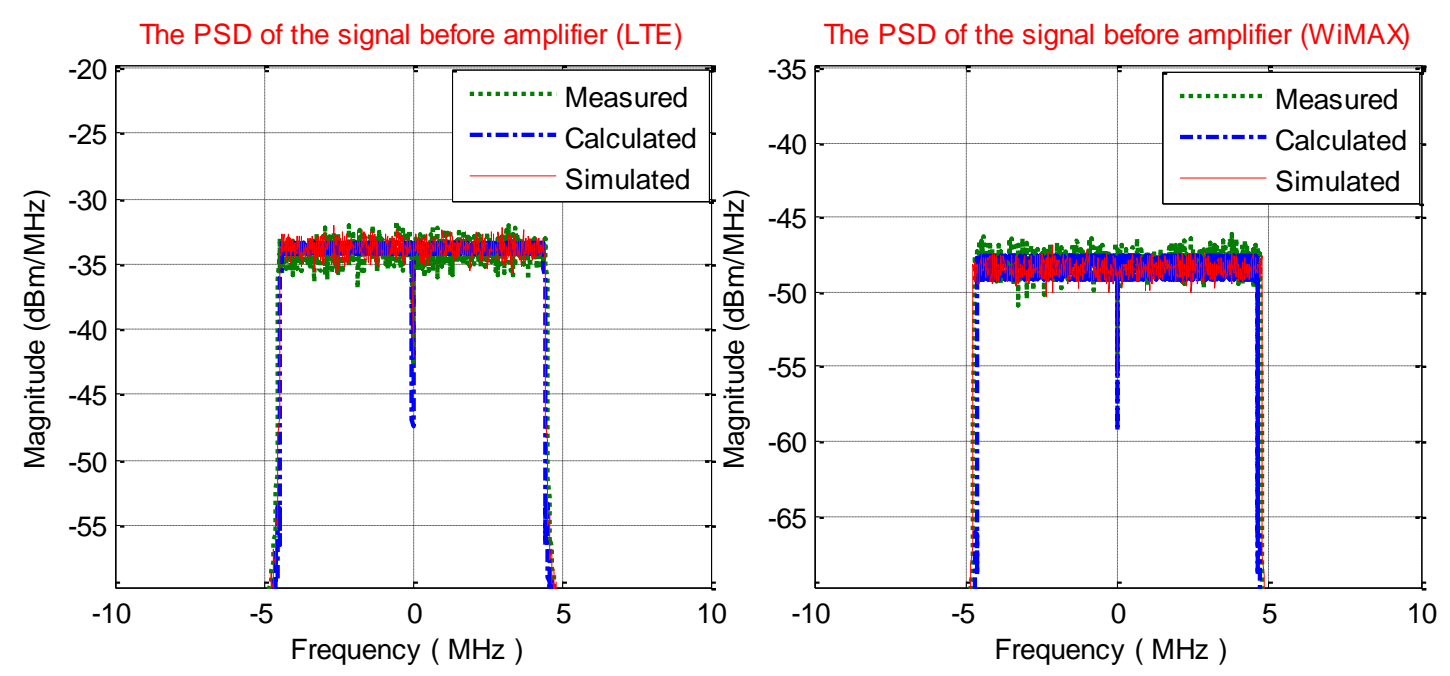

Figure 23 Power spectrum comparison of downlink LTE and WiMAX signals

\subsection{Memoryless Spectrum Regrowth}

The memoryless cases are analyzed in this section.

The low order spectrum regrowth is considered in 4.2.1. Traditionally, PA models only include the 3rd order nonlinearity, which is the most critical when analyzing the distortion. The 3rd order intercept point $\left(I P_{3}\right)$ is usually given in the datasheet of PA or spectrum analyzers.

Apparently, the 3rd order only model is not accurate at describing the spectrum regrowth when the intermodulation is higher than the 3rd order. It becomes necessary to include higher orders in the nonlinear analysis for better precision. The modeling and analysis of spectrum regrowth are shown in 4.2.2. 


\subsubsection{Low Order Spectrum Regrowth}

If only up to the 3rd order nonlinearity is considered in the mathematical model of RF

Power Amplifier's used the Taylor series from (3.9), the coefficient $a_{1}$ is related to the linear gain $G$ of the amplifier, and the coefficient $a_{3}$ is directly related to $I P_{3}$, which can usually be obtained from the manufactures' data sheets of RF power amplifiers. For an amplifier with gain compression $\left(a_{3}<0\right)$,

$$
\begin{aligned}
& a_{1}=10^{\frac{G}{20}} \\
& a_{3}=-\frac{2}{3} 10^{\left(\frac{-I P_{3}}{10}+3 \frac{G}{20}\right)}
\end{aligned}
$$

Since $v(t)=\tilde{v}(t) \cos \left(2 \pi f_{c} t+\theta\right)$, the PSD of $\mathrm{v}(t)$ can be determined by the PSD of equivalent amplified baseband signal $\tilde{y}(t)$ as

$$
P_{v}(f)=\frac{1}{4}\left[P_{\tilde{v}}\left(f-f_{0}\right)+P_{\tilde{v}}\left(f+f_{0}\right)\right]
$$

and then, we need to calculate the PSD of $\tilde{y}(t)$. Using the Wiener-Khintchine Theorem [31],

$$
P_{\tilde{v}}(f)=\int_{-\infty}^{\infty} R_{\tilde{v}}(\tau) e^{-j 2 \pi f \tau} d \tau=F\left\{R_{\tilde{v}}(\tau)\right\}
$$

where $F\{\cdot\}$ is the Fourier Transform of $\{\cdot\}$ and $R_{\widetilde{v}}(\tau)$ is the autocorrelation of $\tilde{v}(t)$. 
The power spectrum $P_{v}(f)$ of $v(t)$ can be presented in terms of the amplifier nonlinear parameter $I P_{3}$, and the linear output power $P_{o}$ of the amplifier as:

$$
\begin{aligned}
P_{v}(f)= & \left(2 P_{0}-12 P_{0}^{2} 10^{-\frac{I P_{3}}{10}}+18 P_{0}^{3} 10^{-\frac{I P_{3}}{5}}\right) \frac{P_{1}\left(f-f_{c}\right)}{N_{s c}} \\
& +\left(48 P_{0}{ }^{3} 10^{-\frac{I P_{3}}{5}}\right) \frac{P_{3}\left(f-f_{c}\right)}{N_{s c}{ }^{3}}
\end{aligned}
$$

where $P_{1}=P_{r}(f), P_{3}=P_{1} \otimes P_{1} \otimes P_{1}$, and $\otimes$ is convolution operator. $P_{o}=\frac{a_{1}{ }^{2} N_{S c}}{4}$ is the linear output power of the amplifier.

Further, if a frequency band is defined by $f_{1}$ and $f_{2}$ outside the passband, the required $I P_{3}$ for a given out-of-band emission level $P_{I M_{3}}\left(f_{1}, f_{2}\right)$ specified in LTE or WiMAX standard can be calculated [7].

We compared the analytical results with the simulation in MATLAB and the measurement made on a real RF power amplifier. The LTE and WiMAX signal were generated by an Agilent E4438C ESG Vector Signal Generator. The carrier frequency was 1.9 GHz. Several measurements of out-of-band emission levels of LTE and WiMAX signals were taken using a CRBAMP-100-6000 power amplifier designed by Crystek. The $I P_{3}$ of this amplifier was $30 \mathrm{dBm}$ with a $20 \mathrm{~dB}$ gain. The resolution bandwidth was chosen as $1 \mathrm{kHz}$. Figure 24 shows the derived amplified spectrum $P_{v}(f)$ compared with the MATLAB simulated spectrum and the spectrum measured from an E4438C ESG 
Vector Signal Analyzer for downlink LTE and WiMAX signals. The simulated and measured RF amplifier spectrum agrees with the analytically predicted spectrum in both the pass-band and shoulder area.
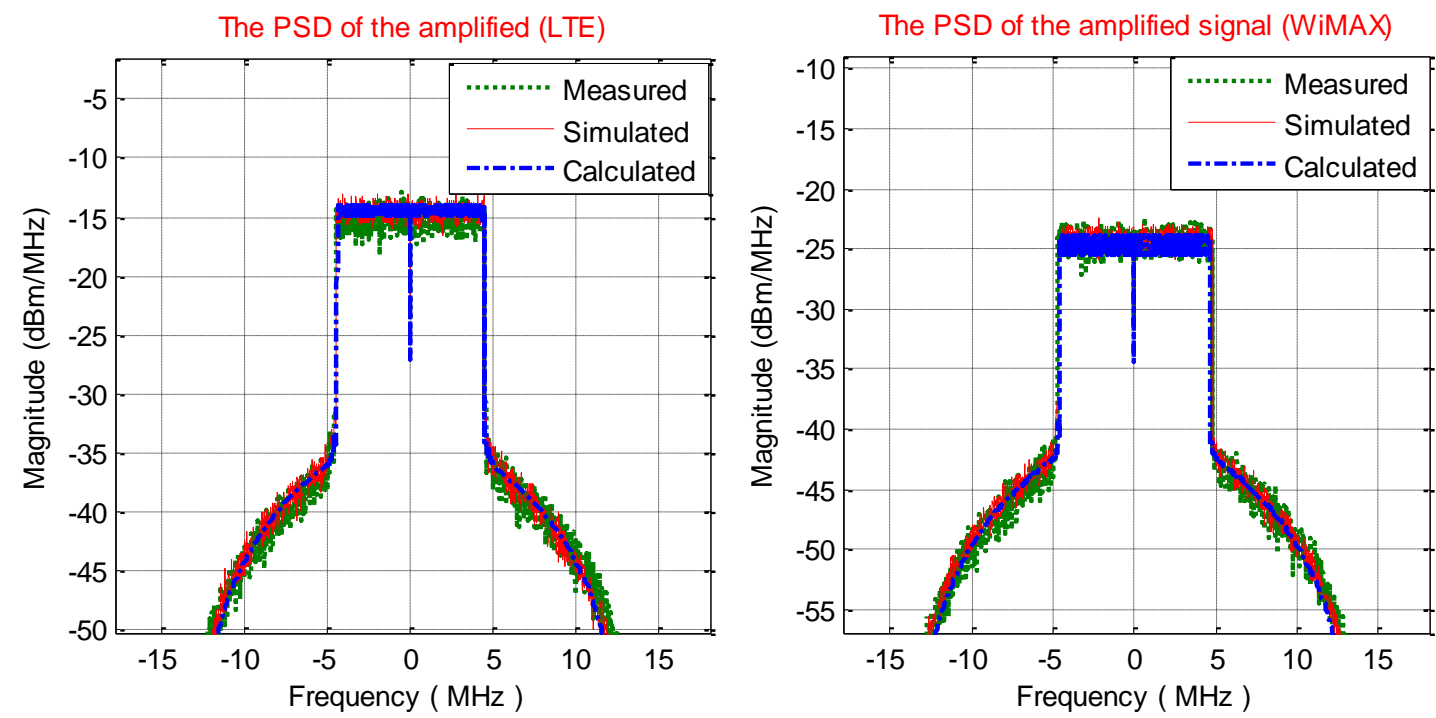

Figure 24 Power spectrum comparison of amplified downlink LTE and WiMAX signals

\subsubsection{High Order Spectrum Regrowth}

\subsubsection{Explicit Expression of High Order Spectrum Regrowth}

The main difficulty to express the power spectrum regrowth to an arbitrarily high order as an explicit form resides in the complicated convolution in (4.7). By modeling the wireless signals as white Gaussian noise with flat power spectrum, this derivation of high order convolution is much easier. As the result, the power spectrum regrowth could be estimated from the intercept points up to an arbitrarily high order as with traditional PA nonlinearity parameter. For CDMA signals, an analytical expression of 
spectrum regrowth is derived by its flat spectrum characteristic, if its statistical property is presented by the Narrow-Band Gaussian Noise (NBGN) model [15].

From the central limit theorem [50], the CDMA signals can be modeled as White Gaussian Noise when the number of chips is very large [15]. The PDF of a CDMA signal was estimated by taking 500 equal width bins between the minimum and maximum value of the modulation envelope and counting the number of occurrence over $2^{14}$ input symbols [51]. The estimated PDF is shown in Figure 25. It is clear that the reverselink CDMA is a Gaussian distribution.

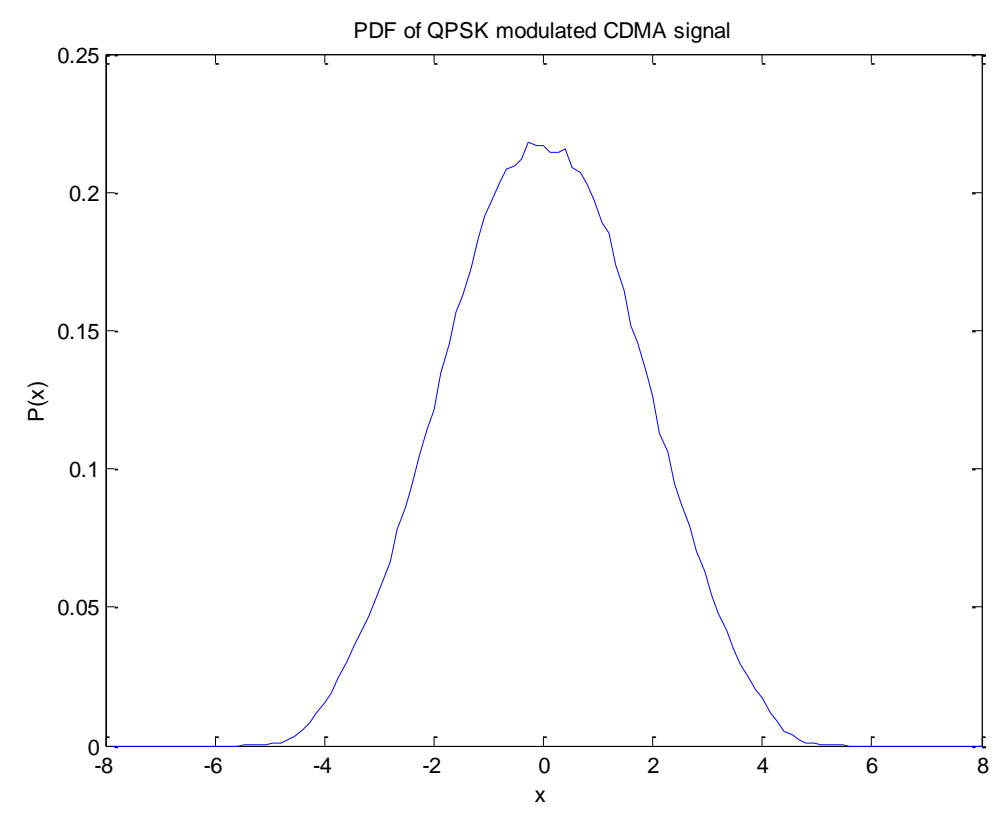

Figure 25 Reverse-link CDMA signal possibility density function

(PDF) estimated from simulated histogram 
Once the CDMA signal is modeled as a bandlimited Gaussian stochastic process with zero mean, it is a bandlimited white Gaussian signal. As the result, the general expression for the power spectrum density (PSD) of a CDMA signal is simply:

$$
P_{f}(f)=\left\{\begin{array}{cc}
\frac{N_{0}}{2}, & |f| \leq B \\
0, & |f|>B
\end{array}\right.
$$

where $B$ is the bandwidth of the signal, $f$ is frequency. This PSD expression of CDMA signal further simplifies derivation for the calculation of spectrum regrowth, which would be otherwise very complicate, if not at all impossible.

With the rectangular shape of power spectrum, [16] used an explicit expression to represent the CDMA spectrum regrowth with polynomial coefficients of PAs up to an arbitrarily high order as

$$
\begin{aligned}
P_{v}(f)= & -\sum_{k=1}^{m}\left[\left(\frac{1}{(2 k-1) !}\left|\sum_{j=0}^{m-k} \frac{(2 j+2 k-1) !}{2^{j} \cdot j !} a_{2 j+2 k-1} N_{o}\right|^{2}\right)\right. \\
& \left.\cdot\left(\frac{1}{(k 2-2) !} \frac{1}{B}\left(\frac{N_{o}}{2}\right)^{2 k-1} \cdot\left\{\sum_{r=0}^{k-q}(-1)^{r}{ }_{r}^{2 k-1} C\left(2 k-2 r-1-\frac{|f|}{B}\right)^{2 k-2}\right\}\right)\right]
\end{aligned}
$$

For $(2 q-3) B \leq|f|<(2 q-1) B, 1 \leq q \leq k$.

For $4 \mathrm{G}$ communication systems, LTE-Advanced signals with large number of subcarriers can be expressed as a white Gaussian noise statistical model with flat power spectrum 
as CDMA. Deriving the explicit expression would help RF designers to predict the PA distortion with simple intercept point expression, and provide insight for digital predistortion design in LTE-Advanced systems.

For statistical model of OFDM-based signals, we have

Theorem: OFDM signals converge to a white Gaussian random process as the number of subcarriers approaches to infinity.

The proof of the theorem is shown in Appendix A. By multi-variant Lindeberg-Feller Theorem [52], the statistical model of OFDM signals converges to a white Gaussian random process as the number of subcarriers approaches infinity. In LTE-Advanced systems with 5 downlink component carrier aggregation, the number of subcarriers is up to 6000 . Therefore, the white Gaussian random process model can be applied to LTEAdvanced signals. The general expression for the power spectrum density (PSD) of a bandlimited LTE-advanced signal is very simple as (4.9). This rectangular power spectrum model can be applied to simplify the derivation of LTE-Advanced spectrum regrowth. Figure 26 shows the shape of the LTE-Advanced power spectrum is approaching to a rectangular when the number of the subcarriers increases. 

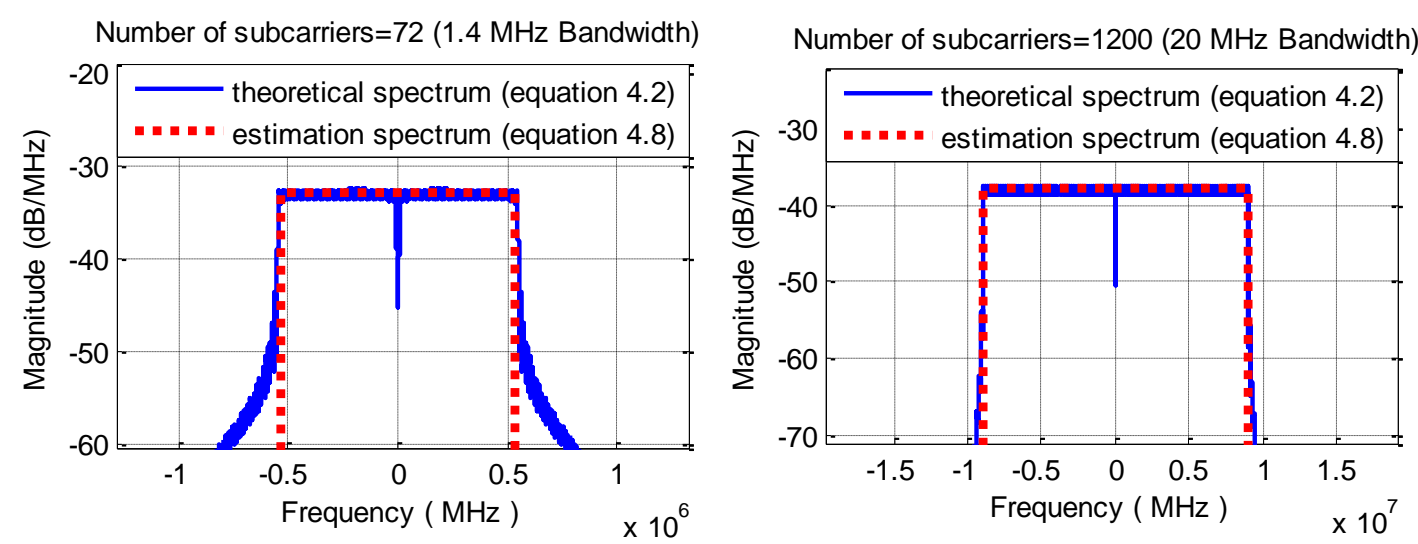

Figure 26 Theoretical spectrum vs estimated spectrum in (4.8)

Due to the white Gaussian noise statistical nature of LTE-advanced signals, if the number of subcarriers is large enough, the power spectrum could also be modeled as the rectangular shape in (4.8). By applying (3.14) and substituting PA's polynomial coefficients $a^{\prime}$ s with the intercept points from our recent result in (4.9), the amplified power spectrum of LTE-advanced signals is

$$
\begin{aligned}
P_{y}(f)=-\sum_{k=1}^{n}( & \left\{\frac{1}{(2 k-1) !} \mid \sum_{j=0}^{n-k} \frac{(2 j+2 k-1) !}{2^{j} \cdot j !} .\right. \\
& \left.\left.\frac{2^{j+k-1}}{\left(\begin{array}{c}
2 j+2 k-1 \\
j+k
\end{array}\right)} 10\left[\frac{-2(j+k-1) I P_{2 j+2 k-1}+(2 j+2 k-1) G}{20}\right]_{N_{o}}\right|^{2}\right\} . \\
& \left\{\frac{1}{(k 2-2) !} \frac{1}{B}\left(\frac{P_{o}}{10^{G / 10}}\right)^{2 k-1} .\right.
\end{aligned}
$$




$$
\begin{aligned}
& \left.\left.\left[\sum_{r=0}^{k-q}(-1)^{r} \cdot{ }_{r}^{2 k-1} C\left(2 k-2 r-1-\frac{|f|}{B}\right)^{2 k-2}\right]\right\}\right) \\
& \text { For }(2 q-3) B \leq|f|<(2 q-1) B, 1 \leq q \leq k
\end{aligned}
$$

Therefore, this result allows designers to specify any orders of spectrum regrowth of LTE-advanced signal amplifiers using simple intercept point expression up to an arbitrarily high order.

\subsubsection{Experimental Analysis}

The analytical result in (4.10) was compared with measurements made on an RF power amplifier. The real LTE-advanced downlink signal was generated by an Agilent E4438C vector signal generator. The carrier frequency is $2000 \mathrm{MHz}$. The resolution bandwidth was chosen as $3 \mathrm{kHz}$. The PA was Mini-Circuits ZFL-1000LN+ with a $13 \mathrm{dBm} \mathrm{IP}$ and a $20 d B$ gain. 


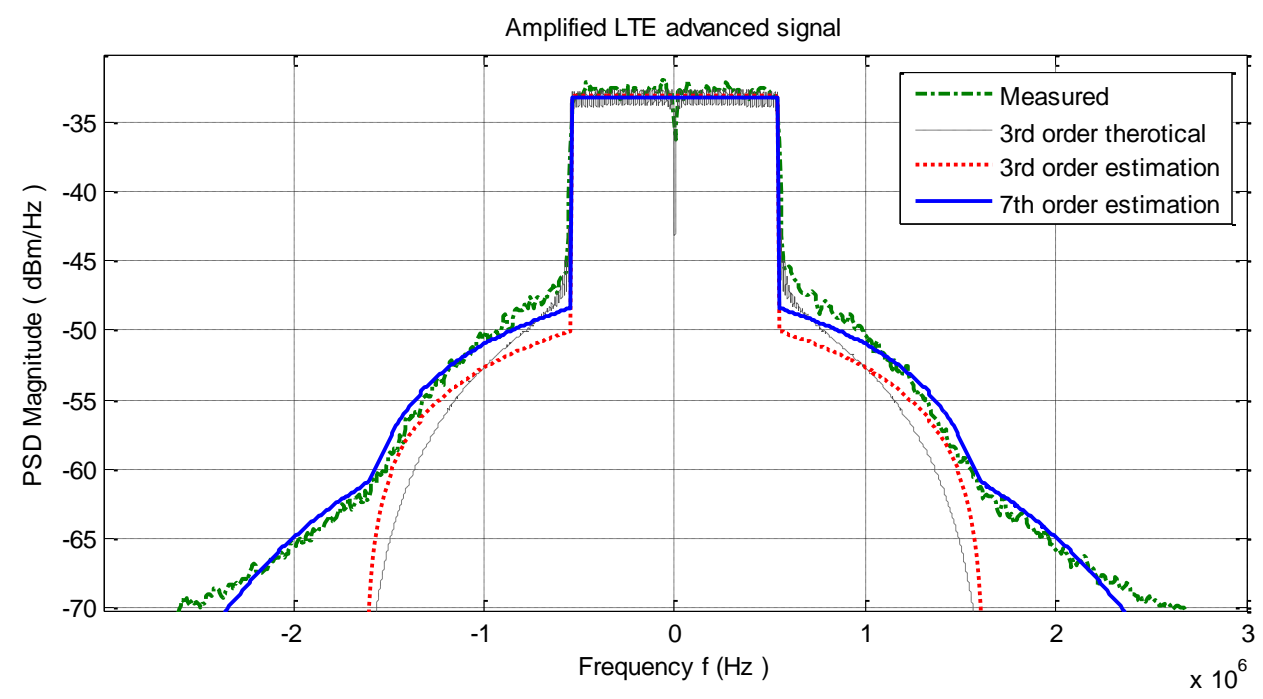

Figure 27 LTE-advanced spectrum regrowth up to 7 th order

In Figure 27, the power spectrum predicted from this analysis is compared with the spectrum measured on an Agilent 89600 vector signal analyzer. No significant difference between the estimated and theoretical results was observed when the 3rd order nonlinearity was applied. It is apparent that the analytically predicted spectrum using only up to the 3rd order cannot fully predict the nonlinearity, but ones with higher order (up to the 7th order) fit the measurement more closely. Slight difference occurs for $P_{o}<-45 \mathrm{dBm}$, but it is comparable to the noise floor. These data verified the analytical results discussed previously.

\subsection{Spectrum Regrowth with Memory Effect}

To illustrate the modeling method, we proposed using a two-tone test to extract the memoryless nonlinearity parameters of the model. The validity test was performed by 
comparing the calculated output from the proposed power amplifier model with the measured output from the PAs run with the same input. The performance of the proposed model can be evaluated by mean square error (MSE) calculation, which presents the output difference between the modeled and the measured time-domain output as

$$
\operatorname{MSE}(d B)=10 \log _{10}\left(\frac{\sum_{n=1}^{N}\left|y(n)-y^{\prime}(n)\right|^{2}}{\sum_{n=1}^{N}|y(n)|^{2}}\right)
$$

Example 1: A 1.4 MHz bandwidth LTE advanced signal was chosen as the PA's input signal. The measurements were made on a ZFL-1000LN+ power amplifier from MiniCircuits with $13 \mathrm{dBm} \mathrm{IP}$ and $20 \mathrm{~dB}$ gain. The comparison of the simulated and the measured spectrum is shown in Figure 28. The nearly symmetrical spectrum indicated that the memory effect was relatively weak. The mean square errors (MSE) were $-39.22 d B$ and $-36.29 d B$ for the proposed and memoryless models respectively. There is no obvious difference between the memory and memoryless models, so considering only the memoryless model was sufficient for this case. Adding memory effect would increase complexity to the model system. 
Amplified 3GPP LTE advanced signal - 1.4 MHz bandwidth

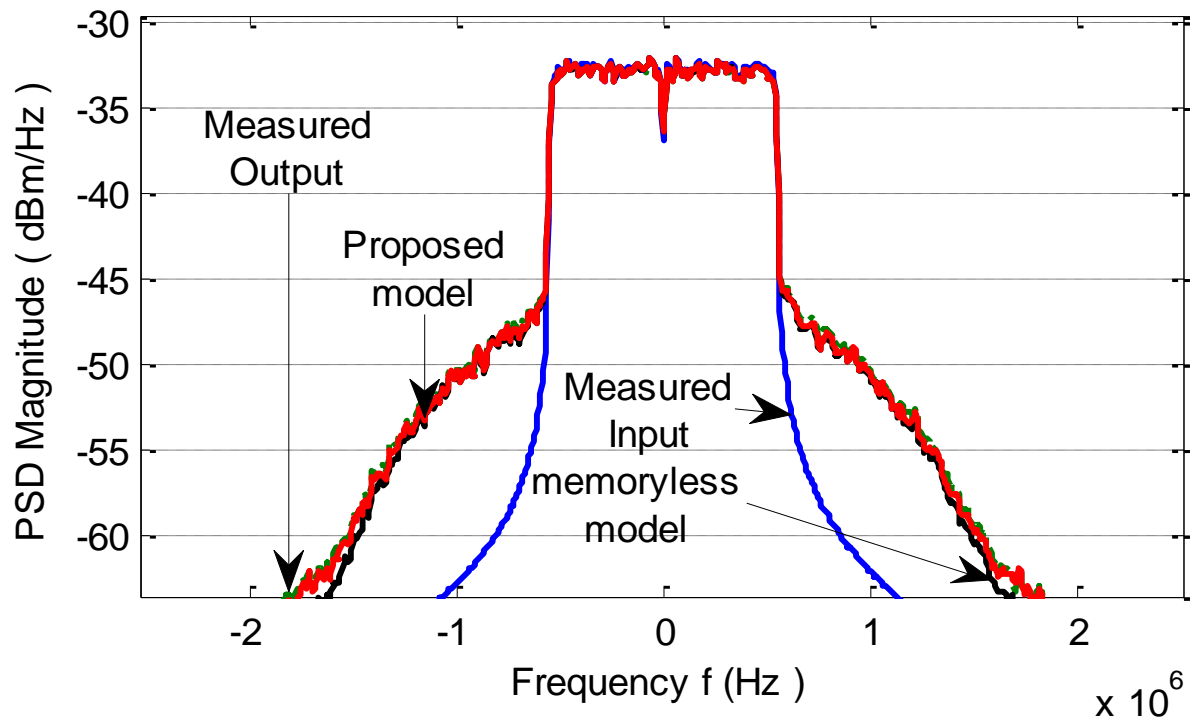

Figure 28 Amplified 1.4 MHz LTE downlink signal

Example 2: A $10 \mathrm{MHz}$ bandwidth LTE advanced signal is used for demonstrating strong memory effect. Measurements were made on a CRBAMP-100-6000 power amplifier from Crystek Corporation, with $30 \mathrm{dBm} \mathrm{IP}_{3}$ and $20 \mathrm{~dB}$ gains. In this example, the LTI portion of the Hammerstein predistorter was assumed to be a pole/zero system with two poles and two zero. The performance of this model is shown in Figure 29. The proposed model agrees with the measured output more closely than the memoryless polynomial model. The mean square errors (MSE) are $-37.22 d B$ and $-16.29 d B$ for the proposed and memoryless models, respectively. The $21 \mathrm{~dB}$ improvement indicates it is necessary to include the memory effect in this PA modeling, and the proposed modeling method is very effective. 


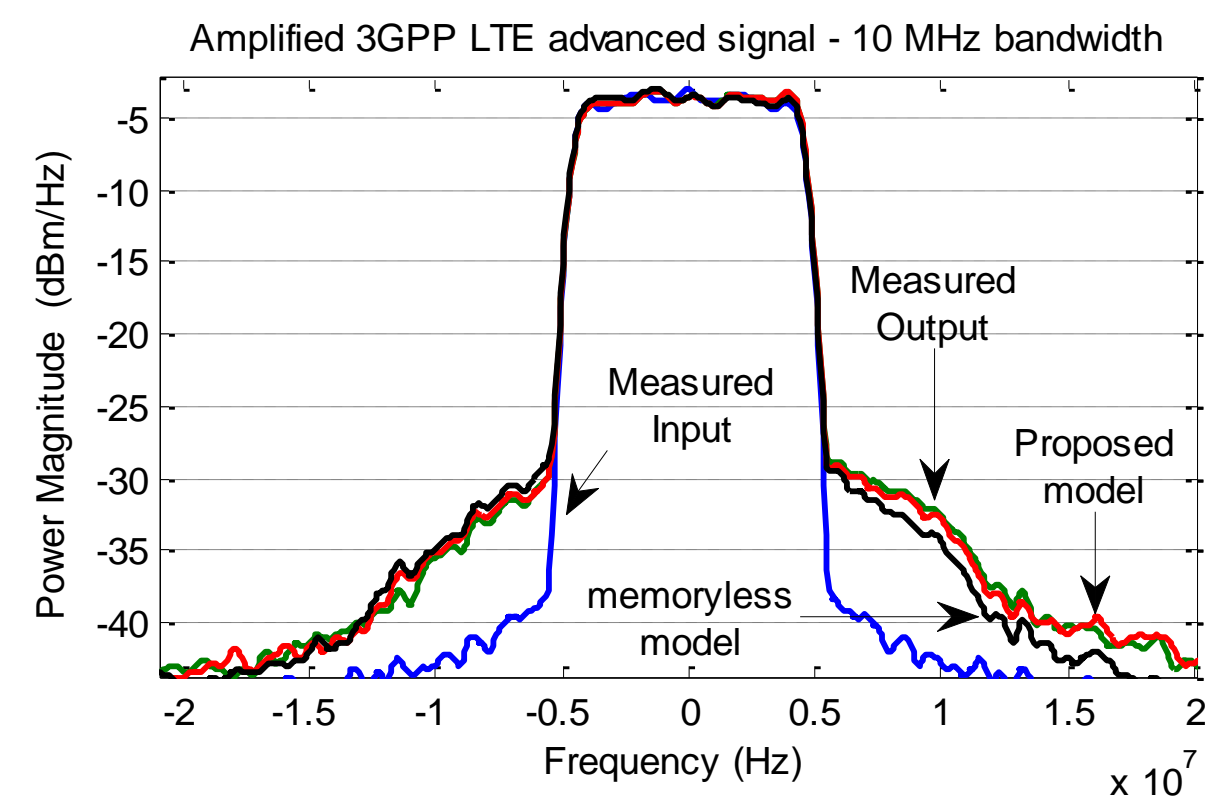

Figure 29 Amplified $10 \mathrm{MHz}$ LTE downlink signal 


\section{Chapter 5 Predistorter Design}

Traditionally, the easiest way to meet the linearity requirements for RF power amplifiers is to use simply the back-off method, but the efficiency is quite low when the Peak-ToAverage Power Ratio (PAPR) is large as in the latest wireless communication systems. Due to its good performance in highly dispersive channels, OFDM has been selected as the modulation scheme in $4 \mathrm{G}$ communication systems. However, compared to singlecarrier systems, OFDM's biggest drawback is its high PAPR [12]. Therefore, the simple back-off method becomes inadequate to linearize PAs in the modern communication systems.

Many new methods, such as Feedback, feed forward, and predistortion, have been proposed to linearize the power amplifiers to obtain linear and efficient devices [53]. Digital Predistortion is one of the most efficient ways among these linearization methods, and it is also the most commonly used method in microwave and satellite applications. Unlike other PA linearization methods, the predistortion methods also provide an improvement near the saturation region [53]. The working theory of predistortion design is shown in Figure 30. A nonlinear block (predistorter) is inserted in front of the PA to generate the reverse characteristic transfer function. The predistorter function is passed through the PA to compensate for the nonlinearity generated from the PA. 

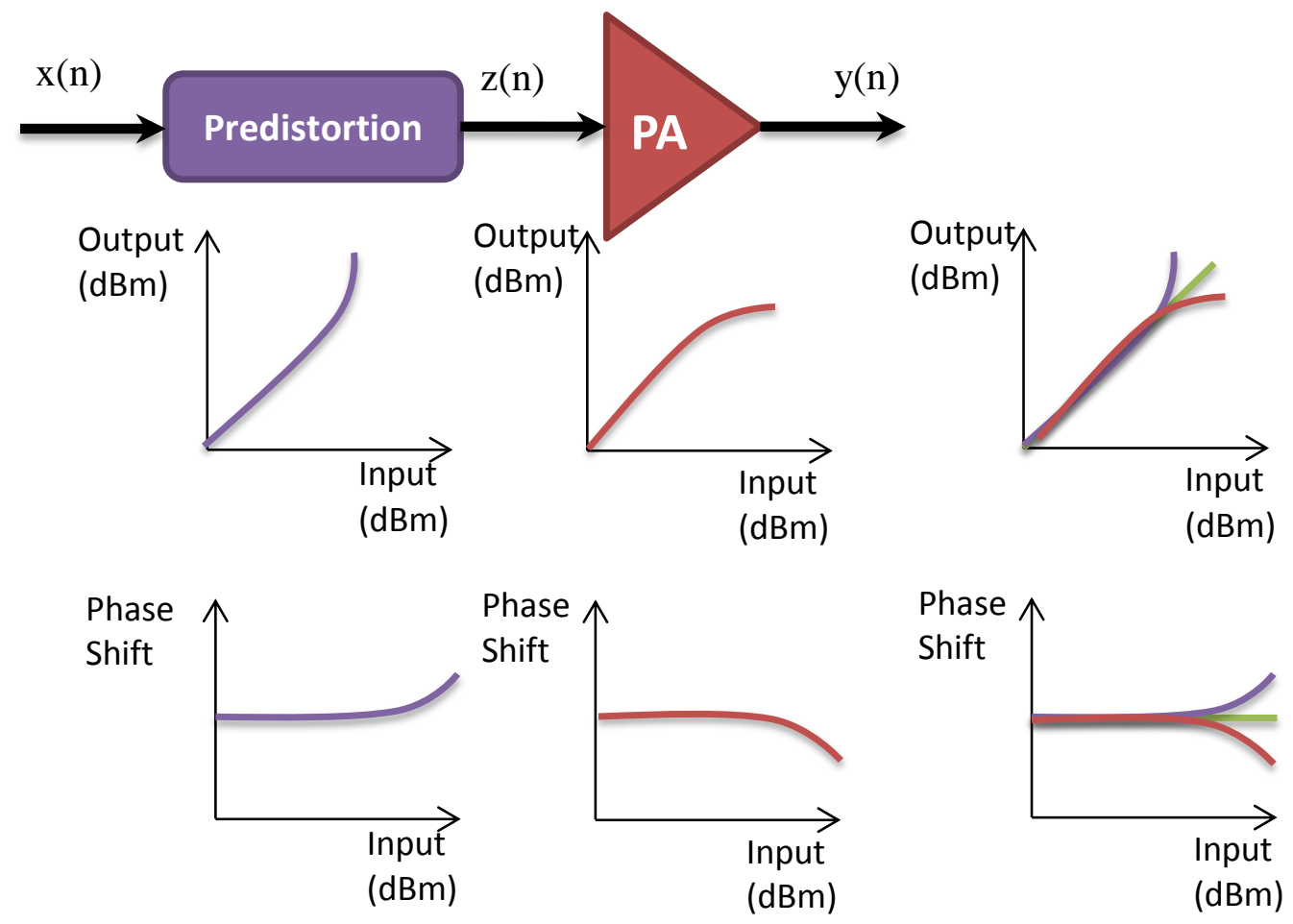

Figure 30 Design theory of predistortion

\subsection{Predistortion Review}

\subsubsection{Memoryless Predistortion}

Although the memory effect caused by the capacitive and inductive devices in the systems with broader bandwidth needs to be considered, it adds complexity to the PA model analysis, especially when PAs do not show much memory effect. Therefore, the predistortion of memoryless PAs is very important, because it can be applied to the predistortion of PAs with memory effect. The Look-Up Table (LUT) based algorithms and the inverse polynomial algorithms are two key algorithms for memoryless models. 


\subsubsection{Look-up Table Predistortion}

For the LUT methods, the inverse characteristic function of the PA is described by the memory contents of a LUT. A traditional LUT is a simple AM/AM and AM/PM predistortion technique. Instead of calculating the values using a mathematical formula, it finds a nearby value from a memory address. The complex gain of the DUT is stored in two look-up tables. The PA model of LUT is given by:

$$
y(n)=z(n) \cdot G[|z(n)|]
$$

where the $G[|z(n)|]$ is the complex gain of the power amplifiers. The AM/AM and AM/PM characteristics of the DUT are derived from the raw measured data using averaging or polynomial fitting techniques.

For the LUT predistorter, the complex envelopes of its input $x(n)$ and output $z(n)$, are related by

$$
z(n)=x(n) \cdot F[|x(n)|]
$$

For any input power, the optimum value of the PD complex gain $F[|x(n)|]$ is determined by equating the composite PD/PA nonlinearity to a nominal constant amplitude gain $K$.

$$
y(n)=x(n) \cdot F[|x(n)|] \cdot G[|x(n) \cdot F[|x(n)|]|]=K \cdot x(n)
$$

Usually, the size of the LUT FPGA works with 512 or 1024 points (power of two). 
LUT algorithm is suitable for processing fast zigzag nonlinear distortion without solving for polynomial coefficients, so it has lower computation complexity and better predistortion performance for memoryless PAs. However, because of size limit of LUTs, there are input signal quantification distortion problems. If the LUT size is increased, the system storage space is enlarged and the convergence speed is therefore reduced. Additionally, the corresponding tanning time is another major drawback [24].

\subsubsection{Inverse Polynomial Predistortion}

Inverse polynomial algorithms approximate the inverse polynomial coefficients of predistorter from a polynomial model of a PA. The theory of pth-order inverse of nonlinear systems [3] was originally used for the compensating the nonlinearities of power amplifiers with memory by a Volterra series model, but this method is unrealistically complicated when higher orders of nonlinearity are taken into account and it is unnecessary if memory effect of a PA is not strong. Sunmin Lim and Changsoo Eun [4] proposed a predistorter for memoryless PAs using a pth-order inverses method. Due to mathematical complexity, only coefficients up to the 9th order of predistorter are given based on a polynomial model of PA up to 9th order.

In 5.2.2, we advanced on the polynomial predistortion method to compensate the memoryless nonlinearity of RF power amplifiers based on a polynomial model, and 
developed a general formula to calculate the coefficients up to a true pth-order. The coefficients of any arbitrarily high order (vs the non-generic 9th order in [4]) of inverse polynomial can be obtained directly from coefficients of a simple polynomial PA model. This makes the process of compensation simpler and complete.

\subsubsection{Predistortion with Memory Effect}

The predistortion design with memory effect is more complex. Two categories are considered for synthesizing the predistortion function, in order to achieve $f_{D P D}\left(\frac{\text { output }}{G}\right)=$ Input. The first category models the PA first, and then inverts the PA model to linearize the PA, shown in Figure 31. The second category is called indirect learning technique initially proposed in [54] shown in Figure 32. It identifies the

predistortion function directly from $\frac{\text { output }}{G}$ and Input relation in time domain. The advantage of this approach is that it eliminates the need for model assumption and parameter estimation of the power amplifier. 


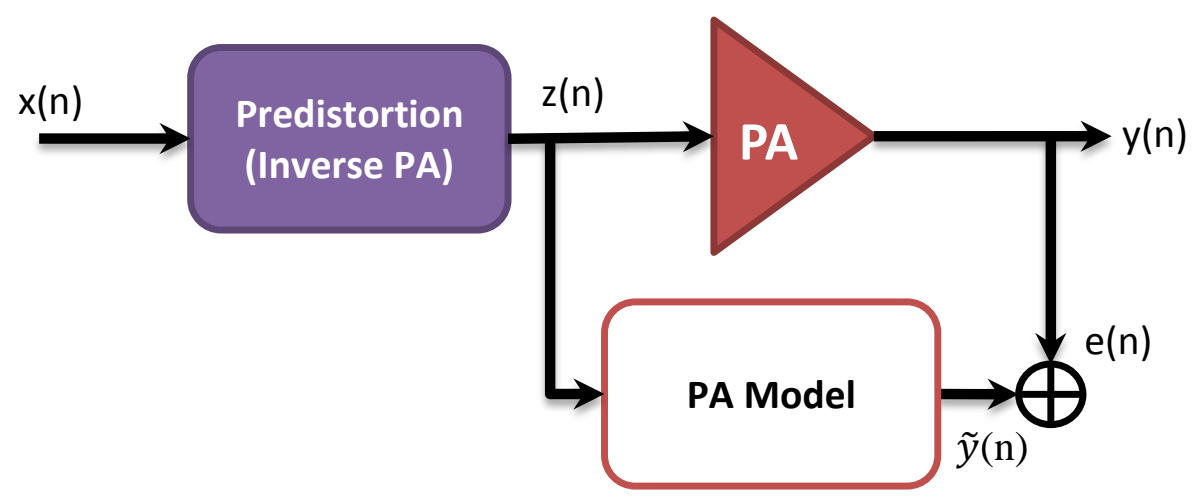

Figure 31 The direct learning architecture for the predistorter

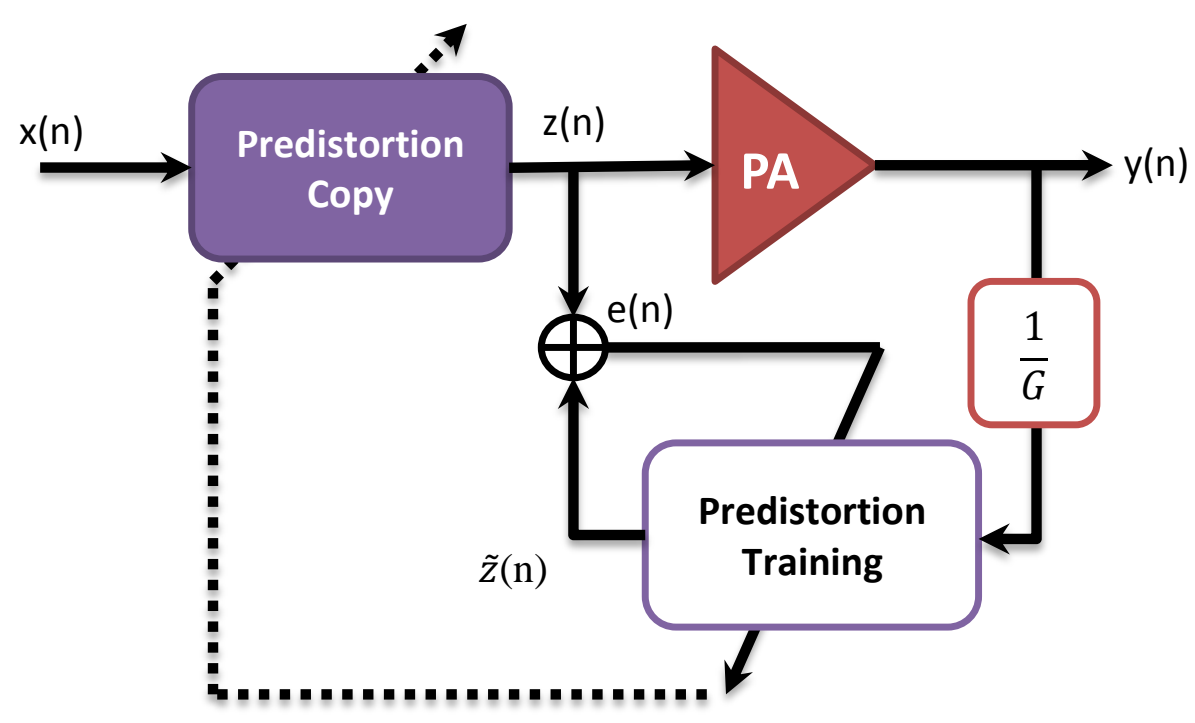

Figure 32 The indirect learning architecture for the predistorter

The indirect learning memory polynomial predistortion model [17] is widely used for PAs/transmitters exhibiting memory effects

$$
z(n)=\sum_{l=0}^{L} \sum_{\substack{m=1 \\ m \text { odd }}}^{M} a_{m, l} y(n-l)|y(n-l)|^{m-1}
$$


where $a_{m, l}$ are the coefficients of the predistorter. Since the model in (5.4) is linear with respect to its coefficients, the predistorter coefficients $a_{m, l}$ can be directly obtained by least-squares. If we define a new sequence as

$$
u_{m, l}(n)=\frac{y(n-l)}{G}\left|\frac{y(n-l)}{G}\right|^{m-1},
$$

The matrix form can be written as:

$$
z=U a
$$

where

$$
\begin{gathered}
z=[z(0), z(1), \ldots, z(N-1)]^{T} \\
U=\left[u_{0, L}, \ldots, u_{0 L}, \ldots, u_{M, 1}, \ldots, u_{M, L}\right]^{T} \\
u_{m, l}=\left[u_{m, l}(0), u_{m, l}(1), \ldots, u_{m, l}(N-1)\right]^{T} \\
a=\left[a_{0, L}, \ldots, a_{0 L}, \ldots, a_{M, 1}, \ldots, a_{M, L}\right]^{T}
\end{gathered}
$$

The least square solution for the coefficients is:

$$
a=\left(U^{H} U\right)^{-1} U^{H} Z
$$

where ()$^{H}$ denotes complex conjugate transpose.

However, two drawbacks affect the performance of the indirect learning model [26]. First, the measurement of PA's output could be noisy, thus, the adaptive algorithm converges to biased values. Second, the nonlinear filters cannot be commuted, i.e., the 
identified adaptive inverse model is actually a post-inverse model. Placing a copy of this model in front of a nonlinear device does not guarantee a good pre-inverse model for the nonlinear device. The authors in [27] compared the indirect and direct learning predistortion methods, and shown that the direct learning architecture achieves a better performance in almost all cases.

Although the LUT is a less complex method, it is nonetheless a memoryless system. It cannot correct PAs memory effects. Some improvements have been made to the LUTs, such as adding linear filters after LUT. Addition of filters allows LUT to compensate not only for nonlinearity, but the memory effects as well, and it is still one order of magnitude less complex than the memory polynomial systems.

\subsection{Proposed Predistorter Design}

\subsubsection{Methodology}

Figure 33 illustrates the proposed DPD learning scheme where is the input signal $x(n)$ to the predistortion unit, whose output $y(n)$ feeds the power amplifier to produce output. RF power amplifier is modeled by two blocks: memoryless nonlinear block (A) and LTI (linear time invariant) block (C) which presents memory effect; Digital Predistorter is modeled by two inverse functions according to PA model: inverse nonlinear block (B) and Inverse LTI block (D). The inverse nonlinear block (B) compensates the memoryless 
distortion of PA modeled by block (A), and inverse LTI block (D) cancels the memory effect present by block (C).

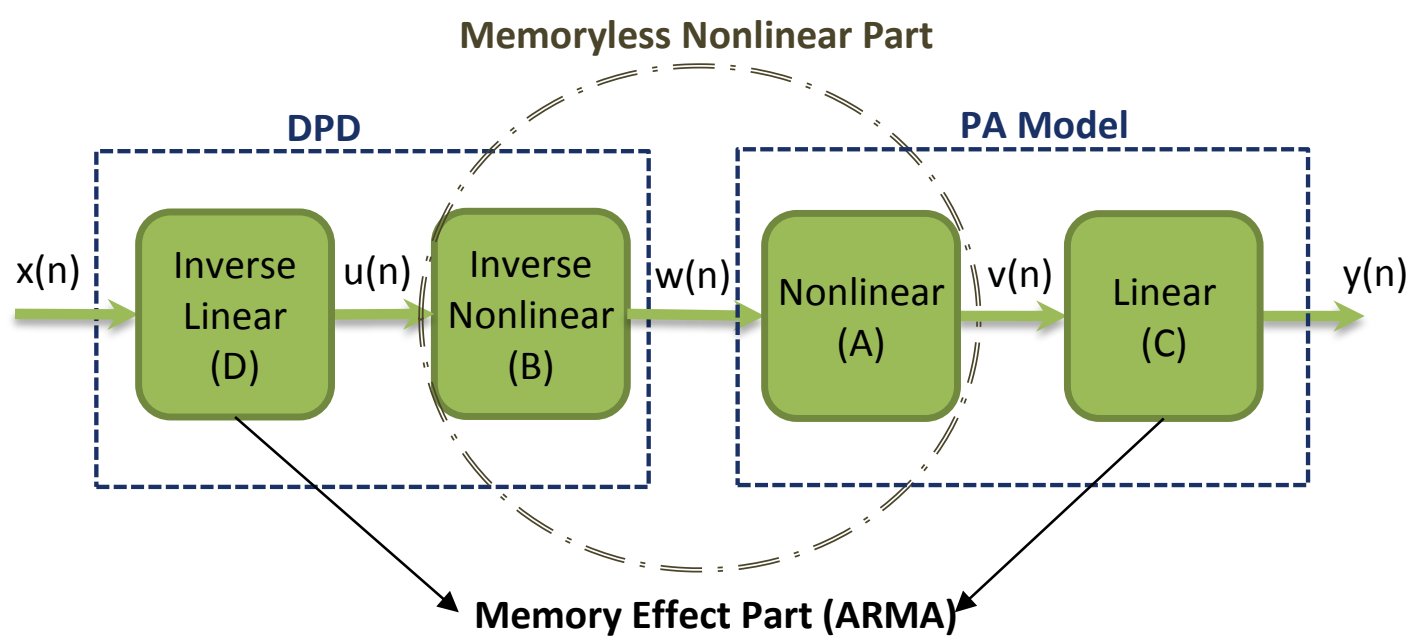

Figure 33 Predistorter learning structure

The PA model we employed here uses a Hammerstein modeling structure, a subsystem shows memoryless nonlinearity followed by a LTI subsystem as the last two blocks in Figure 33.

\subsubsection{Predistortion of Memoryless Nonlinear Part}

Since it is possible to model PAs by polynomial functions, inverse predistortion function (PD) can also be expressed as polynomial.

The inverse polynomial algorithms based on P-th order inverse method [3] is used for predistorting the memoryless nonlinearity of PA. The output of the DPD inverse polynomial Block (B) in Figure 33 can be expressed as 


$$
w(t)=B[u(t)]=\sum_{\substack{p=1 \\ \text { is odd }}}^{P} b_{p} u^{p}(t)=b_{1} u(t)+b_{3} u^{3}(t)+\cdots+b_{P} u^{P}(t),
$$

After substituting $w(t)$ in (5.8), we can rewrite the whole system as

$$
v(t)=H[x(t)]=A\{B[u(t)]\}=h_{1} u(t)+h_{3} u^{3}(t)+\cdots+h_{M \cdot P} u^{M \cdot P}(t) .
$$

The ideal output of a compensated amplifier should have only linear term. Orders of PD and PA polynomials determine maximal order of intermodulation product which can be compensated for. Linearization up to the $p$-th order nonlinearity requires at least $p$-th order polynomial predistorter [4]. From the definition of the p-th order inverse method [3], the coefficients have to be

$$
\begin{gathered}
h_{1}=a_{1}, \\
h_{3}=0, \\
\vdots \\
h_{p}=0,
\end{gathered}
$$

If all the conditions in (5.10) are met, the output of the PA has no nonlinearity up to the p-th of predistorter polynomial order, regardless of the PA's polynomial order. Certain nonlinear terms of orders higher than $p$ may still exist in the compensated output. 
To meet the condition $h_{1}=a_{1}, b_{1}$ has to be equal to 1 . Then starting from $b_{3}$, the coefficient of higher order PD can be calculated from coefficient of lower order PD and the coefficients of PA as

$$
\begin{aligned}
& b_{p}=-\left(\sum_{\substack{\sum_{k=1}^{3} i_{k}=3 \\
i_{k} \text { is odd integer }}}^{\sum_{k=1}^{3} i_{k}=p} b_{i_{1}} b_{i_{2}} b_{i_{3}}\right) \frac{a_{3}}{a_{1}}-\left(\sum_{\substack{\sum_{k=1}^{5} i_{k}=5 \\
i_{k} \text { is odd integer }}}^{\sum_{k=1}^{5} i_{k}=p} b_{i_{1}} b_{i_{2}} b_{i_{3}} b_{i_{4}} b_{i_{5}}\right) \frac{a_{5}}{a_{1}} \\
& -\cdots-\left(\sum_{\substack{\sum_{k=1}^{M} i_{k}=M \\
i_{k} \text { is odd integer }}}^{\sum_{k=1}^{M} i_{k}=p} b_{i_{1}} b_{i_{2}} \ldots b_{i_{M}}\right) \frac{a_{M}}{a_{1}} \\
& =-\sum_{\substack{m=3 \\
m \text { is odd integer }}}^{M}\left[\left(\sum_{\substack{\sum_{k=1}^{m} i_{k}=m \\
i_{k} \text { is odd integer }}}^{\sum_{k=1}^{m} i_{k}=p} b_{i_{1}} b_{i_{2}} \ldots b_{i_{m}}\right) \frac{a_{m}}{a_{1}}\right]
\end{aligned}
$$

where $M$ is the maximum order used for the PA model.

The performance of the inverse polynomial predistorter was demonstrated through both two tone and LTE signals created from Agilent signal studio and uploaded to an Agilent E4438C vector signal generator (VSG). Then the RF signals were applied to the PA ZFL-1000LN+ from Mini-Circuits with $13 \mathrm{dBm} \mathrm{IP} 3$ and a $20 \mathrm{~dB}$ gain, and the measurement was made by an Agilent 89600 vector signal analyzer (VSA). DPD algorithm was computed in MATLAB. The block diagram for the testing is shown in Figure 34. A polynomial PA model with orders up to the 7th was used to test the 
performance of inverse polynomial predistorter. The coefficients of PA model were extracted by two-tone test. Both examples show the proposed inverse polynomial DPD algorithm can effectively linearize the memoryless PA models.

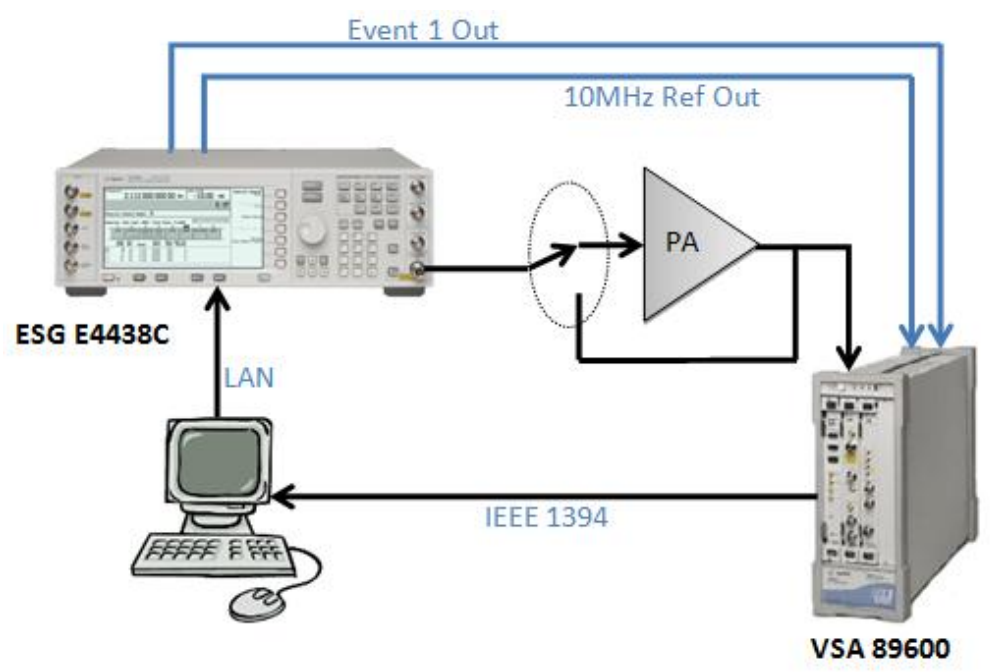

Figure 34 Block diagram for testing

Figure 35 shows the performance of the proposed inverse polynomial predistorter of a two-tone signal. The power spectra of the predistorted signal are compared by choosing different orders of predistorter from 5 to 11 . From the zoom-in plot of the third order intermodulation products comparison, it was observed that the order of the predistorter had to be increased to 11 for the main intermodulation level (3rd order) to be linearized to almost the same level as that of the input signal. Therefore, it is necessary to calculate coefficients of higher order predistorters in order to get better performance of linearization. 

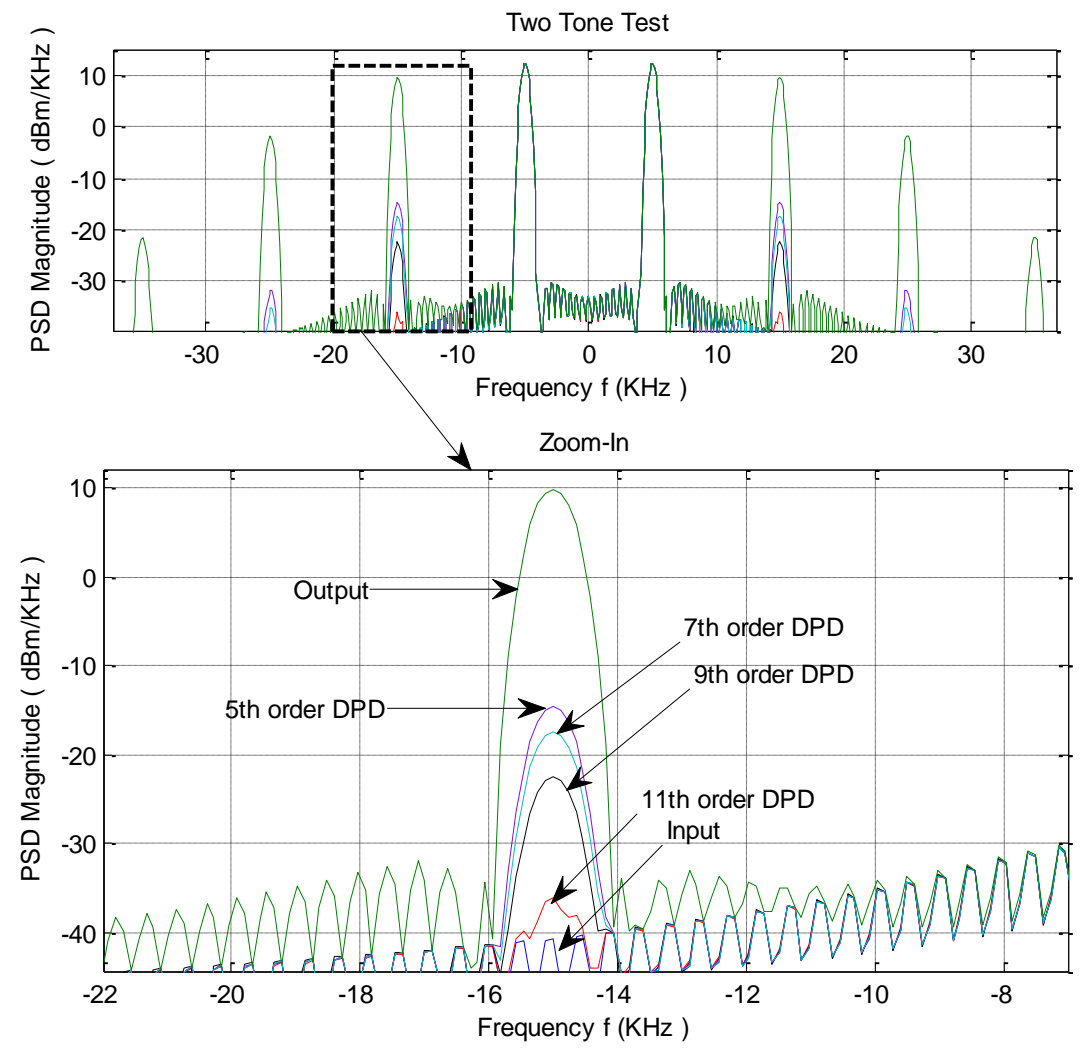

Figure 35 Comparison of the PSDs for two-tone signals

Moreover, 3GPP LTE signal was used here for further experimental verification. Since memory effect was not considered for the proposed model, the bandwidth of LTE downlink signal in this example was chosen to be $1.4 \mathrm{MHz}$. The comparison of the power spectra of the predistorted signal with predistorters of different orders were shown in Figure 36 . The intermodulation product could not be fully removed until the 7th order of the predistorter was used. By applying this DPD algorithm, we greatly reduced the error-vector magnitude (EVM) from $6.50 \%$ to $1.88 \%$, and enhanced the 
Adjacent Channel Leakage power Ratio (ACLR) from $-17.3 d B c$ to $-30.2 d B c$. The constellation using 16QAM before and after DPD comparison is shown in Figure 37.

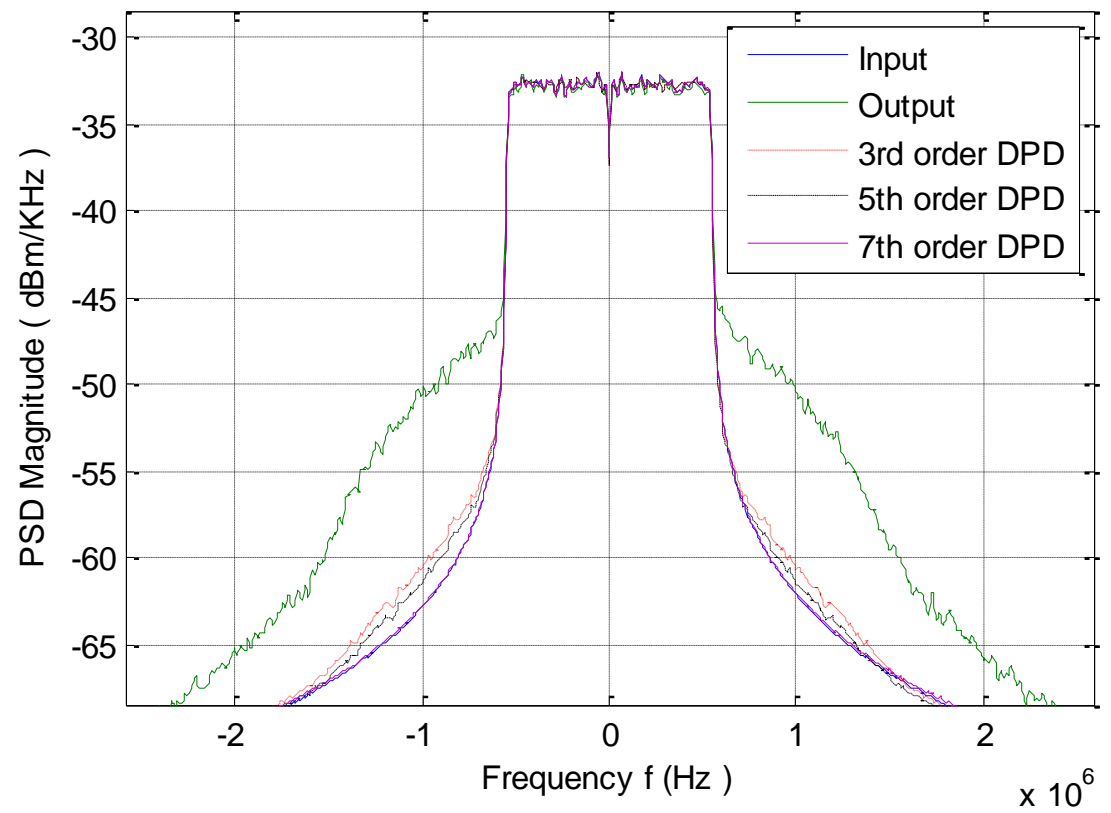

Figure 36 Comparison of the PSDs for LTE signals 


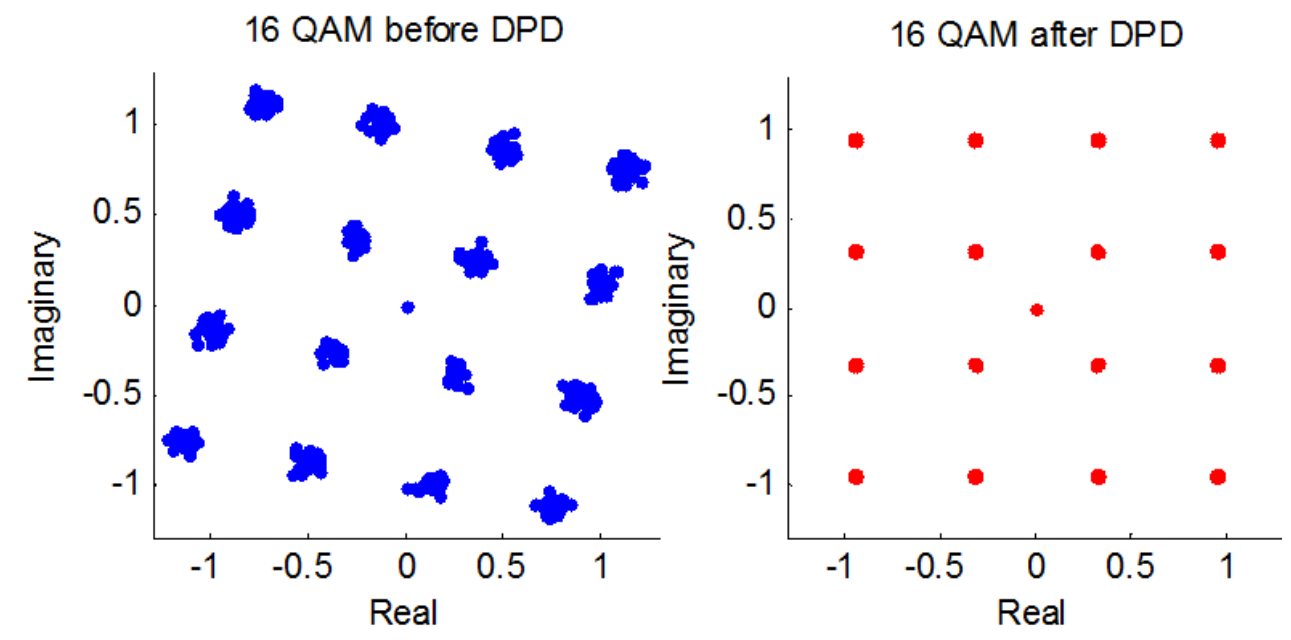

Figure 37 Comparison of the constellation before and after DPD(The phase offset and scatter are canceled and reduced after DPD)

\subsubsection{Predistortion of the Memory Part}

If the combination of the inverse polynomial block $(B)$ and the polynomial block (A) produces linearly amplified signals $v(t)$. The next step is to remove the memory effect of PAs. The memory effect (LTI block C) is modeled as ARMA in (4), so the inverse block (D) is modeled by the same ARMA structure with inverse AR and MA coefficients. This was achieved by exchanging the positions of pole and zero. The output $u(n)$ of inverse ARMA block (D) can be expressed as

$$
\sum_{k=0}^{K} q(k) y(n-k)=\sum_{l=0}^{L} p(l) v(n-l)
$$

This inverse transfer function is:

$$
H(z)=\frac{\sum_{l=0}^{L} p(l) z^{-l}}{\sum_{k=0}^{K} q(k) z^{-k}}
$$


A polynomial PA model with orders up to the 7th was used to test the performance of inverse polynomial predistorter. Two poles and two zero inverse ARMA structures were used for canceling the memory effect. Both EVM (Error Vector Magnitude) and ACPR (Adjacent Channel Power Ratio) were measured to verify the performance of this polynomial ARMA predistorter. Figure 38 shows the EVM performance by choosing various nonlinear order $P$ and various memory order $(K$ or $L)$. It was observed that EVM curve did not drop dramatically when nonlinear order 7 was selected for setting the same memory depth, and when memory order was 2 ( 2 poles and 2 zeros for inverse ARMA) for setting the same nonlinear order. The similar phenomenon happed for ACPR performance in Figure 39. 
EVM vs. Nonlinear Order when memory order is 2

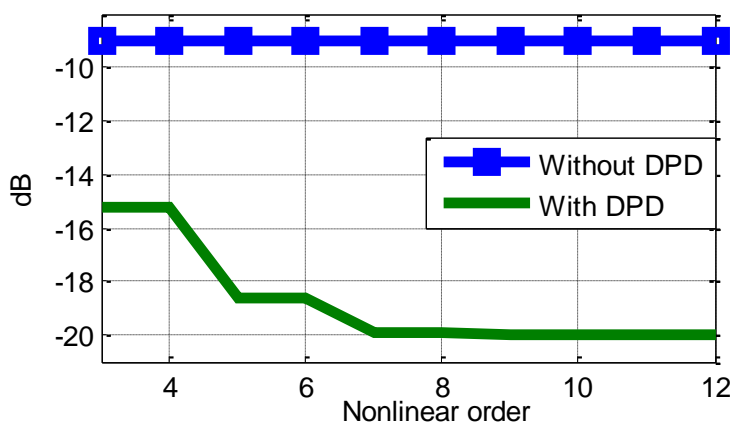

EVM vs. Memory Order when nonlinear order is 7

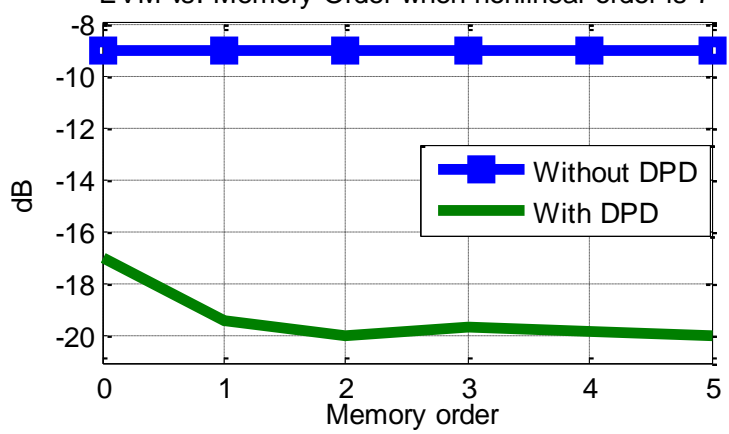

Figure 38 EVM measurements
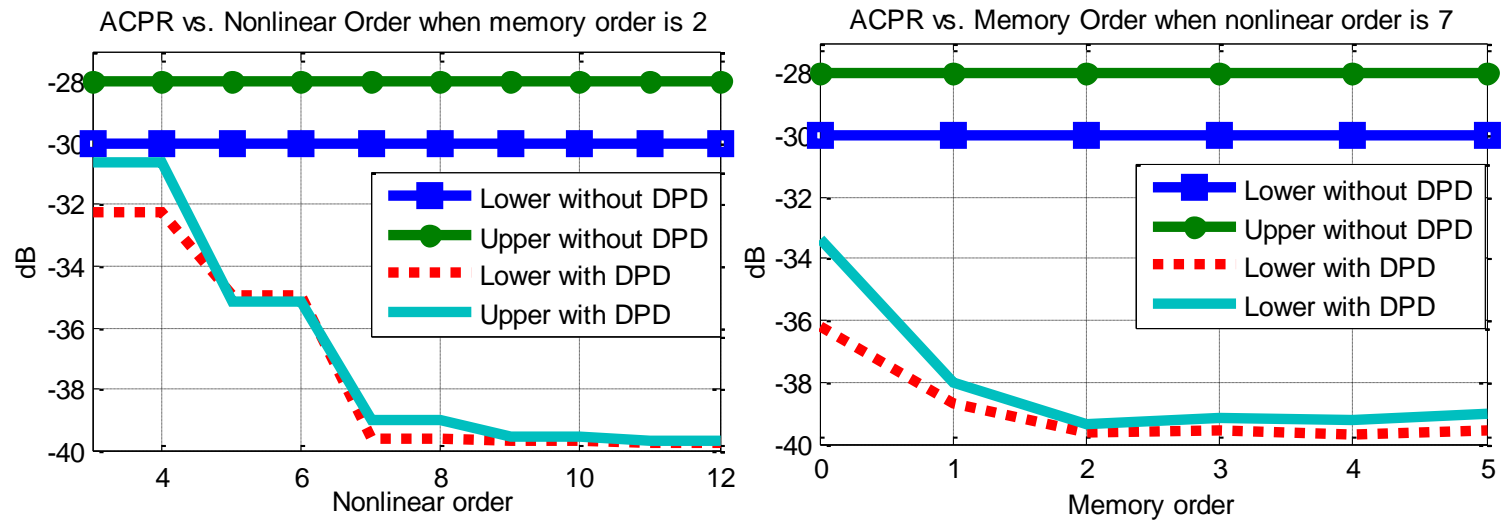

Figure 39 ACPR measurements

The result shows the proposed inverse polynomial DPD algorithm can effectively linearize the PA. The measured power spectrum comparison is shown in Figure 40 . The asymmetric spectrum from the original signal is caused by memory effect. The data shows, when the memory effect of PA is strong, the performance of this polynomial ARMA DPD algorithm is about $8 d B$ better than traditional memoryless DPD at considering memory effects. 


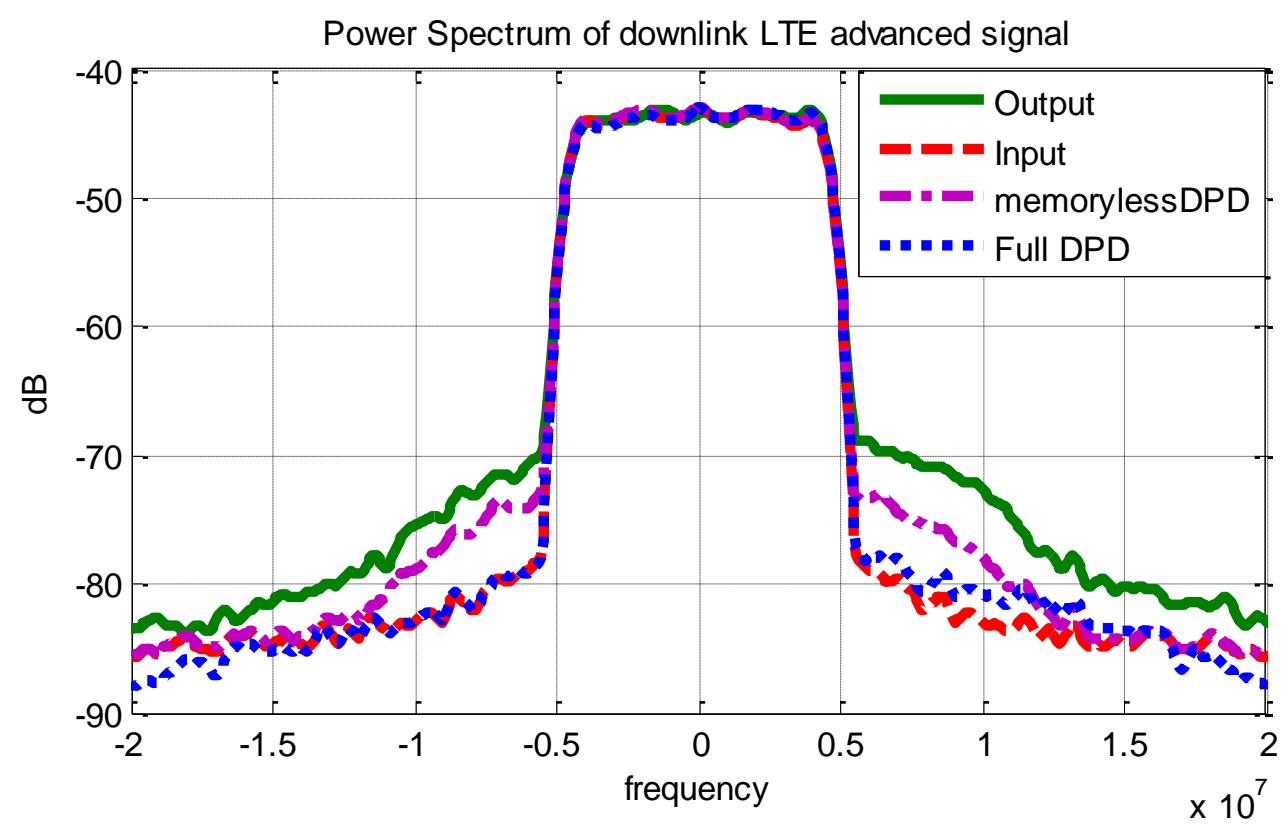

Figure 40 Power Spectrum of downlink LTE advanced signals 


\section{Chapter 6 Conclusion and Future Work}

In this dissertation, a new RF power amplifier model with memory effect is proposed, and the spectrum regrowth in $4 G$ communication systems are analyzed by using different PA model. Then, a simple predistortion algorithm is proposed to reduce both traditional memoryless nonlinear distortion and memory effect. The performance of the new method is demonstrated through experimental measurements. These results helps RF designers predict the spectrum regrowth directly from the characteristic of power amplifiers, and provides an easy yet efficient way for RF designer to linearize the power amplifier.

\subsection{Contributions}

The main contributions of this dissertation are concluded as:

\subsubsection{Low Order Spectrum Regrowth Prediction in 4G Communication Systems}

Based on the relationship between the third order intercept point $\left(I P_{3}\right)$ and the third order polynomial coefficient of an RF power amplifier, our group had previously analyzed and predicted the spectrum regrowth caused by the nonlinearity of RF power amplifiers in relation to their intermodulation parameters for two OFDM based signals: Wi-Fi and digital broadcasting. This technology is extended to LTE and WiMAX signals in this dissertation and verified by the validity of the theoretical result derived by 
experimental data. This provides insight for designing power amplifiers and digital predistorters in terms of out-band spectrum regrowth. (See Chapter 4.2.1)

\subsubsection{Obtain Polynomial Coefficients from Intercept Points of RF Power Amplifiers}

The expression of the polynomial coefficient from the intercept point is generalized to arbitrarily high order. With this result, the memoryless polynomial of each RF power amplifier can be determined from its intercept points or intermodulation products completely and hence could be used for the design of power amplifiers or predistorters. (See Chapter 3.2.1)

\subsubsection{Explicit Expression of High Order Spectrum Regrowth in 4G Communication Systems}

To study the nonlinearity of RF power amplifiers, we showed that LTE-Advanced signals with large number of subcarriers can be expressed as white Gaussian noise statistical model with flat power spectrum. This characteristic enables us to express the spectrum regrowth of LTE-Advanced signals up to an arbitrarily high order in an explicit form, in terms of the traditional PA nonlinearity parameter intercept points without complicated convolution. (See Chapter 4.2.2) 


\subsubsection{Nonlinear Modeling with Memory Effect}

The latest generation wireless communication systems with wider bandwidth, such as Wi-Fi, WiMAX, and LTE, create additional distortion to PAs which affect the performance of digital predistortion (DPD) design. We proposed a simplified Hammerstein structurebased method to analyze the nonlinear characteristic of PAs with consideration of the memory effect. The simplified method produced more accurate results and reduced the complexity of the classic Hammerstein system identification at the same time. (See Chapter 3.2.2)

\subsubsection{High Order Inverse Polynomial Predistortion for Memoryless RF Power}

\section{Amplifiers}

In some applications, there are advantages in using predistorters to linearize PAs before compensating for the memory effect. We presented a polynomial predistortion method based on pth-order inverse method to compensate the memoryless nonlinearity of PAs. The coefficients of the polynomial predistorters up to the arbitrarily high orders can be identified directly from coefficients of the simple polynomial PA model up to arbitrarily high orders, which makes the compensation process much simpler than using complex algorithms. (See Chapter 5.2.2) 


\subsubsection{Predistortion with Memory Effect}

We proposed a predistortion method which uses an inverse polynomial to linearize nonlinearity effect. It also uses an inverse ARMA model to remove memory effect of RF power amplifiers. With this approach, power amplifiers can be predistorted by simply choosing the nonlinearity or memory depth at any arbitrary high orders. (See Chapter $5.2 .3)$

\subsection{Suggestions for Future Research}

The research in this dissertation can be extended in a number of directions, including:

1) Implement the proposed DPD algorithm on DSP or FPGA.

2) Designing an adaptive predistorter based on the proposed DPD structure.

3) Nonlinearity correction of ADC, DAC, mixer, etc.

4) Nonlinearity correction of the whole transceiver. 


\section{References}

[1] Y. Nagata, "Linear amplification technique for digital mobile communications," in IEEE 39th Vehicular Technology Conference, 1989.

[2] J. K.CAVERS, "Amplifier linearization using a digital predistorter with fast adaptation and low memory requirements," IEEE Transactions on Vehicular Technology, vol. 39, no. 4, pp. 374-382, 1990.

[3] M. Schetzen, "Theory of pth-order inverses of nonlinear systems," IEEE Transactions on Circuits and Systems, vol. 23, no. 5, pp. 285- 291, 1976.

[4] S. M. Lim and C. S. Eun, "Predistorter design for a memory-less nonlinear high power amplifier using the pth-order inverse method for OFDM systems," in Proceedings of ISPACS 2005, 2005.

[5] I.-R. R. M.1234, "Requirements related to technical performance for IMT-advanced radio interface(s)," 2008.

[6] S. Parkvall, et al., "LTE-Advanced - Evolving LTE Towards IMT-Advanced," in IEEE Proc. Vehicular Technology Conference, 2008.

[7] C. M. Liu, Spectral Modeling and Nonlinear Distortion Analysis of OFDM Based Wireless LAN Signals, Ph.D. dissertation, Portland State University, 2005.

[8] Q. Wu, H. Xiao, and F. Li, "Linear RF power amplifier design for CDMA signals: A spectrum analysis approach," Microwave Journal, vol. 41, pp. 22-40, 1998.

[9] C. M. Liu, H. Xiao, Q. Wu, and F. Li, "Spectrum modeling of an RF power 
amplifier for TDMA signals," Microwave Journal, vol. 44, pp. 88-109, 2001.

[10] X. Li, C.M. Liu, H. Xiao, and F. Li, "Nonlinearity Analysis of RF Power Amplified TD-SCDMA Signal," in IEEE 10th International Conference on Signal Processing (ICSP), 2010.

[11] H. Xiao, Spectrum modeling for linear RF power amplifier design for digital cellular communication signals, Ph.D. dissertation, Portland State University, 1999.

[12] R. V. Nee and R. Prasad, OFDM for wireless multimedia communications, Boston: Artech House, 2000.

[13] Bahai, A. R., Singh, M., Goldsmith, A. J., \& Saltzberg, B. R., "A new approach for evaluating clipping distortion in multicarrier systems," IEEE journal on Selected areas in communications, vol. 20, no. 5, pp. 1037-1046, 2002.

[14] Ochiai, H., and Imai, H., "On the distribution of the peak-to-average power ratio in OFDM signals," IEEE Trans. Commun., vol. 49, no. 2, pp. 282-289.

[15] X. Li, C. M. Liu, and F. Li, "Statistical Analysis of CDMA and 3G Signal Models," in IEEE ICSP 2012 proceeding, Beijing, 2012.

[16] S. J. Yi, S.W. Nam, S. H. Oh, and J. H. Han, "Prediction of a CDMA output spectrum based on intermodulation products of two-tone test," IEEE Trans. on Microwave Theory and Techniques, vol. 49, pp. 938-946, 2001.

[17] L. Ding, Digital Predistortion of Power Amplifiers for Wireless Applications, Ph.D. thesis,Georgia Institute of Technology, 2004.

[18] W. Bösch and G. Gatti , "Measurement and simulation of memory effects in 
predistortion linearizers," IEEE Trans. Microw. Theory Tech, vol. 37, no. 12, p. 1885-1890, 1989.

[19] X. T. Vuong, and A. F. Guibord, "Modeling of nonlinear elements exhibiting frequency-dependent AM/AM and AM/PM transfer characteristics," Can. Elect. Eng. J., vol. 9, p. 112-116, 1984.

[20] J. Kim and K. Konstantinou, "Digital predistortion of wideband signals based on power amplifier model with memory," Electron. Lett., vol. 37, p. 1417-1418, 2001.

[21] K. J. Muhonen, M. Kavehrad, and R. Krishnamoorthy, "Look-up table technique for adaptive digital predistortion: A development and comparison," IEEE Trans. Veh. Technol., vol. 49, no. 9, p. 1995-2002, 2000.

[22] J. Y. Hassani and M. Kamareei, "Quantization error improvement in a digital predistorter for RF power amplifier linearization," Proc. IEEE Veh. Technol. Conf., vol. 2, p. 1201-1204., 2001.

[23] S. P. Stapleton, G. S. Kandola, and J. K. Cavers, "Simulation and analysis analysis of an adaptive predistorter utilizing a complex spectral convolution," IEEE Trans. Veh. Technol., vol. 41, no. 11, p. 387-394, 1992.

[24] H. Besbes, T. Le-Ngoc, and H. Lin, "A fast adaptive polynomial predistorter for power amplifiers," in Proc. IEEE Global Telecomm. Conf., 2001.

[25] K. C. Lee and P. Gardner, "A novel digital predistorter technique using an adaptive neuro-fuzzy inference system," IEEE Commun. Lett., vol. 7, no. 2, p. 55-57, 2003.

[26] D. Zhou and V. DeBrunner, "A novel adaptive predistorter based on the direct 
learning algorithm," in Proc. IEEE International Conference on Communications, 2004.

[27] H. Paaso and A. Mammela, "Comparison of direct learning and indirect learning predistortion architectures," in ," Wireless Communication Systems. 2008. ISWCS '08. IEEE International Symposium on, 2008.

[28] I. Rosu, "http://www.qsl.net/va3iul/RF\%20Power\%20Amplifiers/RF_Power_Amplifiers.pd f," [Online].

[29] A. M.S., Ahmed, "Analysis, Modelling and Linearization of Nonlinearity and Memory Effects in Power Amplifiers Used for Microwave and Mobile Communications," kassel university press, 2005.

[30] M. J. Franco, Wideband digital predistortion linearization of radio frequency power amplifiers with memory, Ph.D. Thesis, 2005.

[31] L. W.Couch, Digital and Analog Communication Systems, New Jersey: PrenticeHall, Inc., 1996.

[32] T. S. Rappaport, Wireless Communication Principle and Practice, New Jersey: Prentice-Hall, Inc., 1996.

[33] P. Colantonio, F. Giannini, and E. Limiti, High Efficiency RF and Microwave Solid State Power Amplifiers, John Wiley and Sons, Chichester, UK, 2009.

[34] H. AI-Raweshidy, and S. Komaki, Radio over Fiber Technologies for Mobile Communications Networks, Boston, MA: Artech House, 2001. 
[35] S. C. Cripps, Advanced Techniques in RF Power Amplifier Design., Nonvood, MA: Anech House, 2002.

[36] J. Vuolevi, T. Rahkonen, and J. Manninen, "Measurement technique for characterizing memory effects in RF power amplifiers," IEEE Trans. Microwave Theory Tech., vol. 49, no. 8, pp. 1383-1389, 2001.

[37] N. B. Carvalho and J. C. Pedro, "A Comprehensive Explanation of Distortion Sideband Asymmetries," IEEE Trans.on Microwave Theory and Tech., vol. 50, pp. 2090-2101, 2002.

[38] F.M. Ghannouchi and O. Hammi, "Behavioral modeling and predistortion," Microwave Magazine, IEEE, vol. 10, no. 7, pp. 52-64, 2009.

[39] D. Schreurs, M. O'Droma , A. A. Goacher and M. Gadringer, RF power amplifier behavioral modeling, Cambridge Univ. Press, 2008.

[40] E. Eskinat, S. H. Johnson, and W. L. Luyben, "Use of Hammerstein models in identification of nonlinear systems," AIChE J., vol. 37, p. 255-267, 1991.

[41] H. Ku and J.S. Kenney, "Behavioral modeling of nonlinear RF power amplifiers considering memory effects," IEEE Transactions on Microwave Theory and Techniques, vol. 51, no. 12, pp. 2495- 2504, 2003.

[42] C. M. Liu, H. Xiao, Q. Wu, and F. Li, "Spectrum design of RF power amplifier for wireless communication systems,," IEEE Transactions on Consumer Electronics, vol. 48, pp. 72-80, 2002.

[43] X. Li, B. S. Chen, C. M. Liu, X. R. Wang, K. R. Cho, and F. Li, "Spectrum 
Modeling and Regrowth for 4G Wireless Signals," IEE Electronics Letters, vol. 48, no. 4 , p. $244-245,2012$.

[44] S. Benedetto, E. Biglieri, and R. Daffara, "Modeling and performance evaulation of nonlinear satellite links - a Volterra series approach," EEE Trans. Aerosp., Vols. AES-15, p. 494-507, 1979.

[45] E. W. Bai, "An optimal two stage identification algorithm for HammersteinWiener," in Proc. American Contr. Conf, 1998.

[46] X. Li, C.M. Liu, Y. Xu, and F. Li, "Obtaining polynomial coefficients from intercept points of RF power amplifiers," IEE Electronics Letters, vol. 48, no. 19, p. 1238-1240, 2012.

[47] S. Konishi and K. Genshiro, Information criteria and statistical modeling, Springer, 2007.

[48] A. Al-Smadi and A. Al-Zaben, "ARMA model order determination using edge detection: A new perspective," Circuits, systems, and signal processing., vol. 24, no. 6, pp. 723-732., 2005.

[49] A.M. Aibinu, M.J. Salami, A.A. Shafie, and A.R. Najeeb, "Comparing Autoregressive Moving Average (ARMA) coefficients determination using Artificial Neural Networks with other techniques," in Proc. of World Academy of Science,Engineering and Technol, 2008.

[50] W. B. Davenport and W. L. Root, An introduction to the theory of random signals and noise, New York: IEEE Press, 1987. 
[51] M. C. Jeruchim, P. Balaban, K. S. Shanmugan, Simulation of communication systems, Plenum Press, 1992.

[52] R. Durrett, Probability Theories and Examples, Cambridge University Press, 2010.

[53] P.B.Kenington, High Linearity RF Amplifier Design, Norwood, MA: Artech House, 2000.

[54] C. Eun and E. J. Powers, "A new Volterra predistorter based on the indirect learning architecture," IEEE Trans. Signal Process., vol. 45, no. 1, p. 223-227, 1997.

[55] P. Jardin and G. Baudoin, "Filter Lookup Table Method for Power Amplifier Linearization," Vehicular Technology, IEEE Transactions on, vol. 56, no. 3, pp. 1076-1087, 2007. 


\section{Appendix A Proof of the White Gaussian OFDM Theorem in 4.2.2}

An OFDM symbol is constructed as an inverse fast Fourier transform (IFFT) of a set of $a_{k, l}$, which is the modulated transmitted data in the $l$-th OFDM symbol and the $k$-th subcarrier. The mathematical model of an OFDM symbol can be described as [3]

$$
b_{l}(t)=\frac{1}{\sqrt{N_{S c}}} \sum_{k=1}^{N_{S c}} a_{k, l} \cdot e^{j 2 \pi k \Delta f\left(t-T_{C P}\right)}
$$

where $N_{s c}$ is the number of the used subcarriers. The number of subcarriers is up to 1200 in LTE systems, and up to 6000 in LTE-Advanced systems by carrier aggregation of 5 downlink component carriers. $\Delta f$ is the subcarrier frequency spacing. $T_{C P}$ is a guard interval time to create the "circular prefix" to avoid the intersymbol interference (ISI) from the previous symbol. The input data streams are assumed statistically independent and identically distributed in general. A 16 QAM, for instance, $a_{k, l} \in\{-1,1, j,-j,-3,3, j 3,-j 3\}$, the variance $\sigma_{a}^{2}$ of $a_{k, l}$ can be calculated as

$$
\sigma_{a}^{2}=1^{2} \times \frac{1}{4}+(-1)^{2} \times \frac{1}{4}+3^{2} \times \frac{1}{4}+(-3)^{2} \times \frac{1}{4}=5
$$

Rewriting (1) as:

$$
\begin{aligned}
b_{l}(t) & =\frac{1}{\sqrt{N_{S c}}} \sum_{k=1}^{N_{S c}} a_{k, l} \cdot e^{j 2 \pi k \Delta f\left(t-T_{C P}\right)} \\
= & \frac{1}{\sqrt{N_{S c}}} \sum_{k=1}^{N_{S c}} X_{k, l}
\end{aligned}
$$


where $X_{k, l}$ can be considered as random variable. For two different time $t_{1}$ and $t_{2}$ of $X_{k, l}$, we have random vector:

$$
\begin{gathered}
\bar{X}_{k, l}=\left[\begin{array}{l}
X_{k, l}\left(t_{1}\right) \\
X_{k, l}\left(t_{2}\right)
\end{array}\right]=\left[\begin{array}{l}
a_{k, l} \cdot e^{j 2 \pi k \Delta f\left(t_{1}-T_{C P}\right)} \\
a_{k, l} \cdot e^{j 2 \pi k \Delta f\left(t_{2}-T_{C P}\right)}
\end{array}\right] \\
=a_{k, l} \cdot\left[\begin{array}{l}
e^{j 2 \pi k \Delta f\left(t_{1}-T_{C P}\right)} \\
e^{j 2 \pi k \Delta f\left(t_{2}-T_{C P}\right)}
\end{array}\right]
\end{gathered}
$$

Furthermore, the covariance matrix of the random vector in (4) can be calculated as

$$
\Sigma_{\bar{X}}=\sigma_{a}^{2} \cdot\left[\begin{array}{cc}
1 & e^{j 2 \pi k \Delta f\left(t_{1}-t_{2}\right)} \\
e^{j 2 \pi k \Delta f\left(t_{2}-t_{1}\right)} & 1
\end{array}\right]
$$

Therefore, the covariance matrix of the OFDM symbol $b_{l}(\mathrm{t})$ is

$$
\begin{aligned}
& \Sigma_{\left[\begin{array}{l}
b_{l}\left(t_{1}\right) \\
b_{l}\left(t_{2}\right)
\end{array}\right]}=\frac{1}{\sqrt{N_{s c}}} \sum_{k=1}^{N_{s c}} \Sigma_{X}=\sigma_{a}^{2} . \\
& {\left[\begin{array}{cc}
\frac{1}{2} & \frac{1}{N_{s c}} e^{j 2 \pi \Delta f\left(t_{1}-t_{2}\right)} \frac{1-e^{j 2 \pi \frac{N_{s c}}{2} \Delta f\left(t_{1}-t_{2}\right)}}{1-e^{j 2 \pi \Delta f\left(t_{1}-t_{2}\right)}} \\
\frac{1}{N_{s c}} e^{j 2 \pi \Delta f\left(t_{2}-t_{1}\right)} \frac{1-e^{j 2 \pi \frac{N_{S c}}{2} \Delta f\left(t_{2}-t_{1}\right)}}{1-e^{j 2 \pi \Delta f\left(t_{2}-t_{1}\right)}} & \frac{1}{2}
\end{array}\right]}
\end{aligned}
$$

When the number of subcarriers $N_{s c}$ increases to infinity, and $t_{1} \neq t_{2}$,

$$
\Sigma_{\left[\begin{array}{l}
b_{l}\left(t_{1}\right) \\
b_{l}\left(t_{2}\right)
\end{array}\right]} \rightarrow \sigma_{a}^{2} \cdot\left[\begin{array}{cc}
1 & 0 \\
0 & 1
\end{array}\right]
$$


Because of the boundedness of $a_{k, l}$, the Lindeberg-Feller condition is satisfied. By multivariant Lindeberg-Feller Theorem, the statistical model of OFDM signal converges to a white Gaussian random process as the number of subcarriers goes to infinity. 